\title{
Quasistatic evolution of a brittle thin film
}

\author{
Jean-François Babadjian
}

\begin{abstract}
This paper deals with the quasistatic crack growth of a homogeneous elastic brittle thin film. It is shown that the quasistatic evolution of a three-dimensional cylinder converges, as its thickness tends to zero, to a two-dimensional quasistatic evolution associated with the relaxed model. Firstly, a $\Gamma$-convergence analysis is performed with a surface energy density which does not provide weak compactness in the space of Special Functions of Bounded Variation. Then, the asymptotic analysis of the quasistatic crack evolution is presented in the case of bounded solutions that is with the simplifying assumption that every minimizing sequence is uniformly bounded in $L^{\infty}$.
\end{abstract}

Keywords: $\Gamma$-convergence, dimension reduction, thin films, relaxation, quasiconvexity, functions of bounded variation, free discontinuity problems, brittle fracture, quasistatic evolution.

MSC $2000(\mathcal{A M S}):$ 74K30, 49J45, 74K30, 35R35, 49Q20.

\section{Introduction}

Following Griffith's theory of brittle fracture, the variational model of quasistatic crack evolution proposed in [19] is based on the competition between the elastic energy and a surface energy which is necessary to produce a new crack or extending a preexisting one. The classical model was plagued by a few defects, being unable to initiate a crack, or to predict its path during the propagation; the authors were able to overcome these weaknesses, assuming neither a preexisting crack, nor a pre-defined crack path. In their formulation, the time-continuous growth of the cracks is seen as a limit of a discrete time evolution as the time step tends to zero. The first precise mathematical justification of this limit process in the scalarvalued case was given in [18] in the framework of Special Functions of Bounded Variation (SBV). It was subsequently generalized to the vector-valued case in $[13,14]$ (see references therein) in the framework of Generalized Special Functions of Bounded Variation (GSBV). To obtain compactness, the authors had to either add some conservative body and surface loadings with appropriate coerciveness as in [13], or to impose an empirical $L^{\infty}$-boundness hypothesis on every minimizing sequence as in [14]. This latter assumption permits to work in the space $S B V^{p}$ of all $S B V$-functions with $p$-integrable approximate gradient and whose jump set has finite area, in lieu of $G S B V$ (see Definition 1.1 below). The limit crack was defined through a new notion of convergence of rectifiable sets, called $\sigma^{p}$-convergence, related to the notion of jump sets of $S B V$ functions and based on the weak convergence in $S B V^{p}$.

Before dealing with quasistatic evolutions, let us briefly describe the static model. Let $U$ an open subset of $\mathbb{R}^{n}$ representing the reference configuration of a homogeneous elastic material with cracks, whose stored energy density is given by the function $W: \mathbb{R}^{m \times n} \rightarrow \mathbb{R}$, where $\mathbb{R}^{m \times n}$ stands for the set of real $m \times n$ matrices. According to Griffith's theory, the total energy under the deformation $u: U \backslash K \rightarrow \mathbb{R}^{m}$ is given by

$$
\mathcal{E}(u, K):=\int_{U \backslash K} W(\nabla u) d \mathcal{L}^{n}+\mathcal{H}^{n-1}(U \cap K),
$$

where $K$ is an unknown crack. Throughout the paper, $\mathcal{L}^{n}$ and $\mathcal{H}^{n-1}$ denote the $n$-dimensional Lebesgue measure and the $(n-1)$-dimensional Hausdorff measure in $\mathbb{R}^{n}$ respectively (in the sequel, $n$ will always 
be equal to 2 or 3). It is often convenient to use the weak formulation of this problem in the framework of Special Functions of Bounded Variation, replacing the crack $K$ by the jump set $S(u)$ of the deformation $u \in S B V\left(U ; \mathbb{R}^{m}\right)$ and where $\nabla u$ is now the approximate gradient of $u$. We refer to $[2,16]$ for the definitions and basic properties of Functions with Bounded Variation. It is also usual to impose polynomial growth of order $1<p<\infty$ on $W$. Thus, a natural space is the space $S B V^{p}\left(U ; \mathbb{R}^{m}\right)$ of functions $u \in S B V\left(U ; \mathbb{R}^{m}\right)$ such that $\mathcal{H}^{n-1}(S(u) \cap U)<+\infty$ and $\nabla u \in L^{p}\left(U ; \mathbb{R}^{m \times n}\right)$; for such functions, the energy becomes

$$
\mathcal{E}(u):=\mathcal{E}(u, S(u))=\int_{U} W(\nabla u) d \mathcal{L}^{n}+\mathcal{H}^{n-1}(S(u) \cap U) .
$$

Let us now return to the quasistatic evolution. Adding some appropriate boundary conditions and possibly some body and surface loadings, at each time $t \in[0, T]$, we seek to minimize the total energy $(u, K) \mapsto \mathcal{E}(u, K)$ among all legal competitors. Namely, $(u(t), K(t))$ is a minimum energy configuration provided that

$$
\mathcal{E}(u(t), K(t)) \leq \mathcal{E}(u, K)
$$

for every crack $K$ containing $K(t)$ and every deformation $u$, possibly discontinuous across $K$ and satisfying the boundary conditions. More precisely, an irreversible quasistatic evolution of minimum energy configuration is an application $[0, T] \ni t \mapsto(u(t), K(t))$ satisfying the following conditions:

(i) Irreversibility: $K(t)$ increases with $t$;

(ii) Static equilibrium: for every $t \in[0, T]$, the pair $(u(t), K(t))$ is a minimum energy configuration;

(iii) Nondissipativity: the function $t \mapsto \mathcal{E}(u(t), K(t))$ is absolutely continuous.

Condition (iii) express the conservation of the energy in the sense that the derivative of the internal energy $\mathcal{E}(u(t), K(t))$ is equal to the power of the applied forces.

Sometimes a natural small parameter, denoted by $\varepsilon>0$, is involved in the model and one should look at the behavior of the energy and of the quasistatic evolution $\left(u^{\varepsilon}(t), K^{\varepsilon}(t)\right)$ when $\varepsilon$ tends to zero. The notion of $\Gamma$-convergence - see [12] for a complete treatment on that subject - has proved useful in investigating the variational convergence for static problems; in the present context of variational evolution, it remains to see whether such a process is compatible with the evolution. A problem of this type have been studied in [21] where the authors proved a stability result of variational models of quasistatic crack evolution under $\Gamma$-convergence in the antiplanar case. Indeed, they have shown that $\left(u^{\varepsilon}(t), K^{\varepsilon}(t)\right)$ converges, in a certain sense, to a quasistatic evolution of the relaxed model. They had to define a new variational notion of convergence for rectifiable sets that they called $\sigma$-convergence. The limit crack is thus seen as the $\sigma$-limit of $K^{\varepsilon}(t)$ while the limit deformation is nothing but the weak $S B V^{p}(U)$-limit of $u^{\varepsilon}(t)$. In the same spirit, [20] investigates a notion of quasistatic evolution for the elliptic approximation of the Mumford-Shah functional and proves its convergence to a quasistatic growth of brittle fractures in linearly elastic bodies.

In this paper we treat cylindrical bodies whose thickness becomes arbitrarily small and ask how the quasistatic evolution behaves when the small parameter, the thickness of the cylinder, tends to zero. It leads us to perform a 3D-2D asymptotic analysis of a thin film, taking into account the possibility of fracture. An abundant literature exists on dimensional reduction problems and we point the reader to e.g. $[5,9,10,22]$ and references therein.

Let $\Omega_{\varepsilon}:=\omega \times(-\varepsilon, \varepsilon)$ be a three-dimensional cylinder of basis $\omega$, a bounded open subset of $\mathbb{R}^{2}$ and of thickness $2 \varepsilon$, representing the reference configuration of a homogeneous elastic body containing some cracks. The study of the static problem relies on the computation of the $\Gamma$-limit of the sequence of functionals associated to the internal energy, as $\varepsilon$ tends to zero. Namely, we want to look at the behavior of the functional

$$
S B V^{p}\left(\Omega_{\varepsilon} ; \mathbb{R}^{3}\right) \ni u \mapsto \int_{\Omega_{\varepsilon}} W(\nabla u) d \mathcal{L}^{3}+\mathcal{H}^{2}\left(S(u) \cap \Omega_{\varepsilon}\right)
$$




\section{QUASistatic EVolution of A BRITtLe THIN FILM}

when $\varepsilon \rightarrow 0$, in the sense of $\Gamma$-convergence. It is convenient to rescale the problem on the unit cylinder $\Omega:=\Omega_{1}$. Denoting by $x_{\alpha}:=\left(x_{1}, x_{2}\right)$ the in-plane variable, we set $v\left(x_{\alpha}, x_{3} / \varepsilon\right)=u\left(x_{\alpha}, x_{3}\right)$. Replacing $u$ by $v$ in the previous energy, changing variables and dividing by $\varepsilon$, we are led to studying the equivalent sequence of functionals

$$
S B V^{p}\left(\Omega ; \mathbb{R}^{3}\right) \ni v \mapsto \int_{\Omega} W\left(\nabla_{\alpha} v \mid \frac{1}{\varepsilon} \nabla_{3} v\right) d \mathcal{L}^{3}+\int_{S(v) \cap \Omega}\left|\left(\left(\nu_{v}\right)_{\alpha} \mid \frac{1}{\varepsilon}\left(\nu_{v}\right)_{3}\right)\right| d \mathcal{H}^{2},
$$

where, from now on, $\nabla_{\alpha}$ (resp. $\nabla_{3}$ ) will stand for the (approximate) differential with respect to $x_{\alpha}$ (resp. $\left.x_{3}\right), \xi=\left(\xi_{\alpha} \mid \xi_{3}\right)$ for some matrix $\xi \in \mathbb{R}^{3 \times 3}$ and $z=\left(z_{\alpha} \mid z_{3}\right)$ for some vector $z \in \mathbb{R}^{3}$. Such studies have been performed in [5] where the authors imposed to the crack to live on the mid-surface of the plate and also in $[6,9]$. Unlike the latest references, we are working with materials satisfying Griffith's principle. This was not previously taken into account because the authors forced the surface energy to grow linearly with respect to the jump of the deformation. This restriction was due to the application of Integral Representation Theorems in $S B V\left(\Omega ; \mathbb{R}^{3}\right.$ ) (see e.g. Theorem 2.4 in [7] or Theorem 1 in [6]) which hold for such surface energies. Furthermore, it was necessary to obtain a bound in $B V\left(\Omega ; \mathbb{R}^{3}\right)$ of any minimizing sequence. We are convinced that such a behavior is not very realistic because it does not permit to take into account neither Griffith nor Barenblatt theory in which the surface energy behaves asymptotically like a constant: the toughness. However, in [9], Example 2.10, the authors suggest a way to recover a brittle elastic material obeying Griffith's law by a singular perturbation approach (see also [8], Section $8)$.

The originality of our work comes from the fact that we are directly dealing with Griffith surface energy, which was proscribed in $[6,9]$ as explained above. We propose here an alternative proof of this result, deriving the $\Gamma$-limit by an argument very closed to that used in the proof of Theorem 2.1 in [17]. Indeed, to overcome the lack of compactness in $B V\left(\Omega ; \mathbb{R}^{3}\right)$, we use a regular truncation function. It permits us to show that for a deformation belonging to $L^{\infty}\left(\Omega ; \mathbb{R}^{3}\right)$, there is no loss of generality in requiring that any minimizing sequence is uniformly bounded in $L^{\infty}\left(\Omega ; \mathbb{R}^{3}\right)$ and thus, weak $S B V^{p}\left(\Omega ; \mathbb{R}^{3}\right)$-compactness follows. The two-dimensional surface energy remains of Griffith type whereas the bulk energy follows the one obtained in $[6,9]$ : the stored energy density is given by the function $\mathcal{Q} W_{0}: \mathbb{R}^{3 \times 2} \rightarrow \mathbb{R}$ where $W_{0}(\bar{\xi}):=\inf \left\{W(\bar{\xi} \mid z): z \in \mathbb{R}^{3}\right\}$ and $\mathcal{Q} W_{0}$ is the 2D-quasiconvexification of $W_{0}$. Note that we obtain exactly the same energy density as in [22], in which the authors were treating healthy materials, that is without any crack.

Then, it remains to pass to the limit in the quasistatic evolution. We need a notion of convergence of rectifiable sets, in the spirit of $\sigma^{p}$-convergence introduced in [13], but better adapted to the dimensional reduction problems. Intuitively, any limit crack should be a one-dimensional set, that is a two-dimensional set which is invariant by translation in the $x_{3}$ direction. It seems that $\sigma^{p}$-convergence do not permit to obtain such limit cracks. This is why we are led to defining a new notion of convergence (see Definition 4.1) similar to that of $\sigma^{p}$-convergence (see Definition 4.1 in [13]), but for the fact that we impose to any sequence of test functions to have an approximate scaled gradient uniformly bounded in $L^{p}$. Nevertheless it is not sufficient to pass to the limit in the surface term because we need to have compactness for sequences of cracks with bounded scaled surface energy. This difficulty is overcome thanks to Proposition 4.3. The last tool is a Jump Transfer Theorem (see Theorem 2.1 in [18]) stated in a rescaled version in Theorem 4.4 and whose proof uses a De Giorgi type slicing argument.

As in [14], we will assume that any minimizing sequence involved in the quasistatic evolution, remains bounded in $L^{\infty}\left(\Omega ; \mathbb{R}^{3}\right)$ uniformly in $\varepsilon>0$ and in time $t \in[0, T]$ (see (2.8) and the proof of Lemma 5.1). We do not attempt to justify this hypothesis which appears naturally in the scalar case by a truncation argument, whenever the prescribed boundary deformation is also bounded in $L^{\infty}(\Omega \times[0, T] ; \mathbb{R})$, uniformly in $\varepsilon>0$. It will yield weak $S B V^{p}\left(\Omega ; \mathbb{R}^{3}\right)$-compactness and thus allow to define a limit deformation field. Note that we could also have taken the path to add appropriate body and surface loadings, as in [13], with the right order of magnitude, in which case we should be working in a suitable subspace of 
$G S B V\left(\Omega ; \mathbb{R}^{3}\right)$ instead of $S B V^{p}\left(\Omega ; \mathbb{R}^{3}\right)$. We insist on the fact that this boundness hypothesis is completely empirical but has the advantage of avoiding technical difficulties connected with the space $G S B V\left(\Omega ; \mathbb{R}^{3}\right)$.

As mentioned before, we adopt the following

Definition 1.1. For any open set $U \subset \mathbb{R}^{n}$,

$$
S B V^{p}\left(U ; \mathbb{R}^{m}\right):=\left\{w \in S B V\left(U ; \mathbb{R}^{m}\right): \nabla w \in L^{p}\left(U ; \mathbb{R}^{m \times n}\right) \text { and } \mathcal{H}^{n-1}(S(w) \cap U)<\infty\right\} .
$$

Throughout the text, letters as $C$ stand for generic constants which may vary from line to line. We will denote by $\widetilde{\subset}$ and $\cong$ inclusion and equality of sets up to a set of zero $\mathcal{H}^{n-1}$-measure respectively. As for notation, $\mathcal{M}_{b}(U)$ stands for the space of signed Radon measures with finite total variation. If $\mu \in \mathcal{M}_{b}(U)$ and $E$ is a Borel subset of $\mathbb{R}^{n}$, we will denote by $\mu_{\mid E}$ the restriction of the measure $\mu$ to $E$ that is, for every Borel subset $B$ of $\mathbb{R}^{N}, \mu_{\lfloor E}(B)=\mu(E \cap B)$. For any $\mu$-measurable set $A \subset \mathbb{R}^{n}$ and any $\mu$-measurable function $f: \mathbb{R}^{n} \rightarrow \mathbb{R}$, we write $f_{A} f d \mu:=\mu(A)^{-1} \int_{A} f d \mu$ for the average of $f$ over $A$. For the remainder of the paper, strong convergence will always be denoted by $\rightarrow$, whereas weak (resp. weak-*) convergence will be denoted by $\rightarrow$ (resp. $\stackrel{*}{\rightarrow})$. We recall that a sequence $\left\{u_{k}\right\} \subset S B V^{p}\left(U ; \mathbb{R}^{m}\right)$ is said to converge weakly to some $u \in S B V^{p}\left(U ; \mathbb{R}^{m}\right)$, and we write $u_{k} \rightarrow u$ in $S B V^{p}\left(U ; \mathbb{R}^{m}\right)$, if

$$
\left\{\begin{array}{l}
u_{k} \rightarrow u \text { in } L^{1}\left(U ; \mathbb{R}^{m}\right), \\
u_{k} \stackrel{*}{\rightarrow} u \text { in } L^{\infty}\left(U ; \mathbb{R}^{m}\right), \\
\nabla u_{k} \rightarrow \nabla u \text { in } L^{p}\left(U ; \mathbb{R}^{m \times n}\right), \\
\left(u_{k}^{+}-u_{k}^{-}\right) \otimes \nu_{u_{k}} \mathcal{H}_{\left\lfloor S\left(u_{k}\right)\right.}^{n-1} \stackrel{*}{\rightarrow}\left(u^{+}-u^{-}\right) \otimes \nu_{u} \mathcal{H}_{L S(u)}^{n-1} \text { in } \mathcal{M}_{b}\left(U ; \mathbb{R}^{m \times n}\right) .
\end{array}\right.
$$

\section{Formulation of the problem}

\subsection{The physical configuration}

Reference configuration: Let $\omega$ be a bounded open subset of $\mathbb{R}^{2}$ with Lipschitz boundary and define $\Omega_{\varepsilon}:=\omega \times(-\varepsilon, \varepsilon)$. The cylinder $\bar{\Omega}_{\varepsilon}$ denotes the reference configuration of a homogeneous elastic body with cracks, whose stored energy density is given by the function $W: \mathbb{R}^{3 \times 3} \rightarrow \mathbb{R}$. We assume $W$ to be a $\mathcal{C}^{1}$ and quasiconvex function satisfying standard $p$-growth and $p$-coercivity conditions $(1<p<\infty)$ : there exists $\beta>0$ such that

$$
\frac{1}{\beta}|\xi|^{p}-\beta \leq W(\xi) \leq \beta\left(1+|\xi|^{p}\right), \text { for all } \xi \in \mathbb{R}^{3 \times 3} .
$$

Let $p^{\prime}=(p-1) / p$ be the conjugate exponent of $p$. In particular (see [11]), the derivative of $W$, denoted by $\partial W$, satisfies some $(p-1)$-growth condition, namely

$$
|\partial W(\xi)| \leq \beta\left(1+|\xi|^{p-1}\right), \text { for all } \xi \in \mathbb{R}^{3 \times 3} .
$$

Boundary conditions: Let $\omega^{\prime} \subset \mathbb{R}^{2}$ be such that $\bar{\omega} \subset \omega^{\prime}$ and $\Omega_{\varepsilon}^{\prime}:=\omega^{\prime} \times(-\varepsilon, \varepsilon)$ be the enlarged cylinder. We submit the body to a "smooth" given deformation

$$
G^{\varepsilon} \in W^{1,1}\left([0, T] ; W^{1, p}\left(\Omega_{\varepsilon}^{\prime} ; \mathbb{R}^{3}\right)\right)
$$

on the lateral boundary $\partial \omega \times(-\varepsilon, \varepsilon)$ of the cylinder $\Omega_{\varepsilon}$. For homogeneity, we assume that $G^{\varepsilon}$ satisfies

$$
\left\|G^{\varepsilon}\right\|_{W^{1,1}\left([0, T] ; W^{1, p}\left(\Omega_{\varepsilon}^{\prime} ; \mathbb{R}^{3}\right)\right)} \leq C \varepsilon^{1 / p} .
$$


QUASISTATIC EVOLUTION OF A BRITTLE THIN FILM

As in $[14,18]$, we express the lateral boundary condition at time $t$ by requiring that $U=G^{\varepsilon}(t)$ a.e. on $\left[\omega^{\prime} \backslash \bar{\omega}\right] \times(-\varepsilon, \varepsilon)$ whenever $U \in S B V^{p}\left(\Omega_{\varepsilon}^{\prime} ; \mathbb{R}^{3}\right)$ is a kinematically admissible deformation field. Since the Dirichlet boundary condition is only prescribed on the lateral boundary, we do not need, as in [14, 18], to extend the whole cylinder but only its lateral part, which can be trivially done by extending the base domain $\omega$ to $\omega^{\prime}$. As a consequence, any admissible crack will necessarily be contained in $\bar{\omega} \times(-\varepsilon, \varepsilon)$. We will assume that the remainder of the boundary, $\omega \times\{-\varepsilon, \varepsilon\}$, is traction free so that the prescribed boundary deformation is the only driving mechanism. Since the admissible cracks never run into $\omega \times\{-\varepsilon, \varepsilon\}$, there is no need, in contrast to $[14,18]$, to remove the part $\omega \times\{-\varepsilon, \varepsilon\}$ from the surface energy of the crack, so that the surface energy of a crack associated to a test function $V \in S B V^{p}\left(\Omega_{\varepsilon}^{\prime} ; \mathbb{R}^{3}\right)$ will be exactly $\mathcal{H}^{2}(S(V))$.

Initial conditions: As in [18], we consider a body without any preexisting crack (see Remark 5.4). If $U_{0}^{\varepsilon} \in S B V^{p}\left(\Omega_{\varepsilon}^{\prime} ; \mathbb{R}^{3}\right)$ is a given initial deformation satisfying $U_{0}^{\varepsilon}=G^{\varepsilon}(0)$ a.e. on $\left[\omega^{\prime} \backslash \bar{\omega}\right] \times(-\varepsilon, \varepsilon)$, we suppose that the Griffith equilibrium condition is satisfied, that is $U_{0}^{\varepsilon}$ minimizes

$$
V \mapsto \int_{\Omega_{\varepsilon}} W(\nabla V) d x+\mathcal{H}^{2}(S(V))
$$

among $\left\{V \in S B V^{p}\left(\Omega_{\varepsilon}^{\prime} ; \mathbb{R}^{3}\right): \quad V=G^{\varepsilon}(0)\right.$ a.e. on $\left.\left[\omega^{\prime} \backslash \bar{\omega}\right] \times(-\varepsilon, \varepsilon)\right\}$.

Quasistatic evolution: If we follow word for word the arguments developed in [13, 14], applying them to $\omega^{\prime} \times(-\varepsilon, \varepsilon)$ in place of what is used in those references, namely $\Omega_{\varepsilon}^{\prime \prime}$, a Lipschitz extension of $\omega \times(-\varepsilon, \varepsilon)$ with $\bar{\omega} \times[-\varepsilon, \varepsilon] \subset \Omega_{\varepsilon}^{\prime \prime}$, we get the existence of a crack $K^{\varepsilon}(t) \subset \bar{\omega} \times(-\varepsilon, \varepsilon)$ increasing in time and a deformation field $U^{\varepsilon}(t) \in S B V^{p}\left(\Omega_{\varepsilon}^{\prime} ; \mathbb{R}^{3}\right)$ such that

- $U^{\varepsilon}(0)=U_{0}^{\varepsilon}, K^{\varepsilon}(0) \cong S\left(U_{0}^{\varepsilon}\right)$ and $U^{\varepsilon}(0)$ minimizes

$$
V \mapsto \int_{\Omega_{\varepsilon}} W(\nabla V) d x+\mathcal{H}^{2}(S(V))
$$

among $\left\{V \in S B V^{p}\left(\Omega_{\varepsilon}^{\prime} ; \mathbb{R}^{3}\right): \quad V=G^{\varepsilon}(0)\right.$ a.e. on $\left.\left[\omega^{\prime} \backslash \bar{\omega}\right] \times(-\varepsilon, \varepsilon)\right\}$;

- for any $t \in(0, T], S\left(U^{\varepsilon}(t)\right) \widetilde{\subset} K^{\varepsilon}(t)$ and $U^{\varepsilon}(t)$ minimizes

$$
V \mapsto \int_{\Omega_{\varepsilon}} W(\nabla V) d x+\mathcal{H}^{2}\left(S(V) \backslash K^{\varepsilon}(t)\right)
$$

among $\left\{V \in S B V^{p}\left(\Omega_{\varepsilon}^{\prime} ; \mathbb{R}^{3}\right): \quad V=G^{\varepsilon}(t)\right.$ a.e. on $\left.\left[\omega^{\prime} \backslash \bar{\omega}\right] \times(-\varepsilon, \varepsilon)\right\} ;$

- the total energy

$$
E_{\varepsilon}(t):=\int_{\Omega_{\varepsilon}} W\left(\nabla U^{\varepsilon}(t)\right) d x+\mathcal{H}^{2}\left(K^{\varepsilon}(t)\right)
$$

is absolutely continuous with respect to the time $t$ and

$$
E_{\varepsilon}(t)=E_{\varepsilon}(0)+\int_{0}^{t} \int_{\Omega_{\varepsilon}} \partial W\left(\nabla U^{\varepsilon}(\tau)\right) \cdot \nabla \dot{G}^{\varepsilon}(\tau) d x d \tau .
$$

\subsection{The rescaled configuration}

As usual in dimension reduction, we perform a scaling so as to study an equivalent problem stated on a cylinder of unit thickness $\Omega:=\omega \times I$, where $I:=(-1,1)$. Let $\Omega^{\prime}:=\omega^{\prime} \times I$ be the enlarged rescaled cylinder. 


\section{JEAN-FranÇOIS BABADJian}

Boundary conditions (rescaled): Let $g^{\varepsilon}\left(t, x_{\alpha}, x_{3}\right)=G^{\varepsilon}\left(t, x_{\alpha}, \varepsilon x_{3}\right)$ be the rescaled boundary deformation. Changing variables, (2.3) implies that

$$
\left\|g^{\varepsilon}\right\|_{W^{1,1}\left([0, T] ; L^{p}\left(\Omega^{\prime} ; \mathbb{R}^{3}\right)\right)}+\left\|\left(\nabla_{\alpha} g^{\varepsilon} \mid \frac{1}{\varepsilon} \nabla_{3} g^{\varepsilon}\right)\right\|_{W^{1,1}\left([0, T] ; L^{p}\left(\Omega^{\prime} ; \mathbb{R}^{3 \times 3}\right)\right)} \leq C .
$$

In fact, we will further restrict $g^{\varepsilon}$ in (2.7) by requiring the strong convergence of both $g^{\varepsilon}$ and its scaled gradient together with a uniform bound in $L^{\infty}\left(\Omega^{\prime} \times[0, T] ; \mathbb{R}^{3}\right)$. In particular, the limit $g \in$ $W^{1,1}\left([0, T] ; W^{1, p}\left(\Omega^{\prime} ; \mathbb{R}^{3}\right)\right)$ satisfies $\nabla_{3} g=0$. In the sequel, we will identify the space $S B V^{p}\left(\omega ; \mathbb{R}^{3}\right)$ (resp. $\left.W^{1, p}\left(\omega ; \mathbb{R}^{3}\right), L^{p}\left(\omega ; \mathbb{R}^{3}\right)\right)$ with those functions $v \in S B V^{p}\left(\Omega ; \mathbb{R}^{3}\right)\left(\right.$ resp. $W^{1, p}\left(\Omega ; \mathbb{R}^{3}\right), L^{p}\left(\Omega ; \mathbb{R}^{3}\right)$ ) such that $D_{3} v=0$ in the sense of distributions. As a consequence $g \in W^{1,1}\left([0, T] ; W^{1, p}\left(\omega^{\prime} ; \mathbb{R}^{3}\right)\right)$.

Initial conditions (rescaled): Let $u_{0}^{\varepsilon}\left(x_{\alpha}, x_{3}\right)=U_{0}^{\varepsilon}\left(x_{\alpha}, \varepsilon x_{3}\right)$ the rescaled initial deformation, then $u_{0}^{\varepsilon} \in S B V^{p}\left(\Omega^{\prime} ; \mathbb{R}^{3}\right), u_{0}^{\varepsilon}=g^{\varepsilon}(0)$ a.e. on $\left[\omega^{\prime} \backslash \bar{\omega}\right] \times I$ and $u_{0}^{\varepsilon}$ minimizes

$$
v \mapsto \int_{\Omega} W\left(\nabla_{\alpha} v \mid \frac{1}{\varepsilon} \nabla_{3} v\right) d x+\int_{S(v)}\left|\left(\left(\nu_{v}\right)_{\alpha} \mid \frac{1}{\varepsilon}\left(\nu_{v}\right)_{3}\right)\right| d \mathcal{H}^{2},
$$

among $\left\{v \in S B V^{p}\left(\Omega^{\prime} ; \mathbb{R}^{3}\right): \quad v=g^{\varepsilon}(0)\right.$ a.e. on $\left.\left[\omega^{\prime} \backslash \bar{\omega}\right] \times I\right\}$.

Quasistatic evolution (rescaled): We set

$$
u^{\varepsilon}\left(t, x_{\alpha}, x_{3}\right)=U^{\varepsilon}\left(t, x_{\alpha}, \varepsilon x_{3}\right) \text { and } \Gamma^{\varepsilon}(t)=\left\{x \in \bar{\omega} \times I: \quad\left(x_{\alpha}, \varepsilon x_{3}\right) \in K^{\varepsilon}(t)\right\} .
$$

Then, $u^{\varepsilon}(t) \in S B V^{p}\left(\Omega^{\prime} ; \mathbb{R}^{3}\right)$ and $\Gamma^{\varepsilon}(t) \subset \bar{\omega} \times I$ is increasing in time. Moreover,

- $u^{\varepsilon}(0)=u_{0}^{\varepsilon}, \Gamma^{\varepsilon}(0) \cong S\left(u_{0}^{\varepsilon}\right)$;

- for all $t \in(0, T], S\left(u^{\varepsilon}(t)\right) \widetilde{\subset} \Gamma^{\varepsilon}(t)$ and $u^{\varepsilon}(t)$ minimizes

$$
v \mapsto \int_{\Omega} W\left(\nabla_{\alpha} v \mid \frac{1}{\varepsilon} \nabla_{3} v\right) d x+\int_{S(v) \backslash \Gamma^{\varepsilon}(t)}\left|\left(\left(\nu_{v}\right)_{\alpha} \mid \frac{1}{\varepsilon}\left(\nu_{v}\right)_{3}\right)\right| d \mathcal{H}^{2},
$$

among $\left\{v \in S B V^{p}\left(\Omega^{\prime} ; \mathbb{R}^{3}\right): \quad v=g^{\varepsilon}(t)\right.$ a.e. on $\left.\left[\omega^{\prime} \backslash \bar{\omega}\right] \times I\right\} ;$

- the total energy

$$
\mathcal{E}_{\varepsilon}(t):=\int_{\Omega} W\left(\nabla_{\alpha} u^{\varepsilon}(t) \mid \frac{1}{\varepsilon} \nabla_{3} u^{\varepsilon}(t)\right) d x+\int_{\Gamma^{\varepsilon}(t)}\left|\left(\left(\nu_{\Gamma^{\varepsilon}(t)}\right)_{\alpha} \mid \frac{1}{\varepsilon}\left(\nu_{\Gamma^{\varepsilon}(t)}\right)_{3}\right)\right| d \mathcal{H}^{2}
$$

is absolutely continuous in time and

$$
\mathcal{E}_{\varepsilon}(t)=\mathcal{E}_{\varepsilon}(0)+\int_{0}^{t} \int_{\Omega} \partial W\left(\nabla_{\alpha} u^{\varepsilon}(\tau) \mid \frac{1}{\varepsilon} \nabla_{3} u^{\varepsilon}(\tau)\right) \cdot\left(\nabla_{\alpha} \dot{g}^{\varepsilon}(\tau) \mid \frac{1}{\varepsilon} \nabla_{3} \dot{g}^{\varepsilon}(\tau)\right) d x d \tau .
$$

We would like to perform an asymptotic analysis of this quasistatic evolution when the thickness $\varepsilon$ tends to zero. To this end, we start by stating a $\Gamma$-convergence result in order to guess how the energy is behaving through the dimensional reduction. In fact, we will prove in Section 3 that the functional

$$
S B V^{p}\left(\Omega ; \mathbb{R}^{3}\right) \ni v \mapsto \int_{\Omega} W\left(\nabla_{\alpha} v \mid \frac{1}{\varepsilon} \nabla_{3} v\right) d x+\int_{S(v)}\left|\left(\left(\nu_{v}\right)_{\alpha} \mid \frac{1}{\varepsilon}\left(\nu_{v}\right)_{3}\right)\right| d \mathcal{H}^{2}
$$


QUASISTATIC EVOLUTION OF A BRITTLE THIN FILM

$\Gamma$-converges for the strong $L^{1}\left(\Omega ; \mathbb{R}^{3}\right)$-topology to

$$
S B V^{p}\left(\omega ; \mathbb{R}^{3}\right) \ni v \mapsto 2 \int_{\omega} \mathcal{Q} W_{0}\left(\nabla_{\alpha} v\right) d x_{\alpha}+2 \mathcal{H}^{1}(S(v)),
$$

where $W_{0}: \mathbb{R}^{3 \times 2} \rightarrow \mathbb{R}$ is defined by $W_{0}(\bar{\xi}):=\inf \left\{W(\bar{\xi} \mid z): z \in \mathbb{R}^{3}\right\}$,

$$
\mathcal{Q} W_{0}(\bar{\xi}):=\inf _{\varphi \in W_{0}^{1, \infty}\left(Q^{\prime} ; \mathbb{R}^{3}\right)} \int_{Q^{\prime}} W_{0}\left(\bar{\xi}+\nabla_{\alpha} \varphi\left(x_{\alpha}\right)\right) d x_{\alpha}
$$

is the 2D-quasiconvexification of $W_{0}$ and $Q^{\prime}:=(0,1)^{2}$ is the unit square of $\mathbb{R}^{2}$. A two-dimensional quasistatic evolution relative to the boundary data $g(t)$ for the relaxed model is an application $[0, T] \ni$ $t \mapsto(u(t), \gamma(t))$ such that $u(t) \in S B V^{p}\left(\omega^{\prime} ; \mathbb{R}^{3}\right), u(t)=g(t)$ a.e. on $\omega^{\prime} \backslash \bar{\omega}, \gamma(t) \subset \bar{\omega}$ and the three following properties hold:

(i) Irreversibility: $\gamma\left(t_{1}\right) \widetilde{\subset} \gamma\left(t_{2}\right)$, for every $0 \leq t_{1} \leq t_{2} \leq T$;

(ii) Minimality: $S(u(0)) \cong \gamma(0), u(0)$ minimizes

$$
v \mapsto 2 \int_{\omega} \mathcal{Q} W_{0}\left(\nabla_{\alpha} v\right) d x_{\alpha}+2 \mathcal{H}^{1}(S(v)),
$$

among $\left\{v \in S B V^{p}\left(\omega^{\prime} ; \mathbb{R}^{3}\right): \quad v=g(0)\right.$ a.e. on $\left.\omega^{\prime} \backslash \bar{\omega}\right\}$ and, for every $t \in(0, T], S(u(t)) \widetilde{\subset} \gamma(t)$ and $u(t)$ minimizes

$$
v \mapsto 2 \int_{\omega} \mathcal{Q} W_{0}\left(\nabla_{\alpha} v\right) d x_{\alpha}+2 \mathcal{H}^{1}(S(v) \backslash \gamma(t)),
$$

among $\left\{v \in S B V^{p}\left(\omega^{\prime} ; \mathbb{R}^{3}\right): \quad v=g(t)\right.$ a.e. on $\left.\omega^{\prime} \backslash \bar{\omega}\right\}$;

(iii) Nondissipativity: The total energy

$$
\mathcal{E}(t):=2 \int_{\omega} \mathcal{Q} W_{0}\left(\nabla_{\alpha} u(t)\right) d x_{\alpha}+2 \mathcal{H}^{1}(\gamma(t))
$$

is absolutely continuous in time and

$$
\mathcal{E}(t)=\mathcal{E}(0)+2 \int_{0}^{t} \int_{\omega} \partial\left(\mathcal{Q} W_{0}\right)\left(\nabla_{\alpha} u(\tau)\right) \cdot \nabla \dot{g}(\tau) d x_{\alpha} d \tau .
$$

Note that the previous equality makes sense because we will prove in Proposition 4.7 that $\mathcal{Q} W_{0}$ is of class $\mathcal{C}^{1}$ provided $W$ is also of class $\mathcal{C}^{1}$. The main result of this paper is that the three-dimensional quasistatic evolution converges, in the sense detailed below, towards a two-dimensional quasistatic evolution associated with the $\Gamma$-limit model. This is formally expressed as the following

Theorem 2.1. For all $\varepsilon>0$, let $[0, T] \ni t \mapsto\left(u^{\varepsilon}(t), \Gamma^{\varepsilon}(t)\right)$ be a three-dimensional (rescaled) quasistatic evolution relative to the boundary data $g^{\varepsilon}(t)$. Assume that

$$
\left\{\begin{array}{l}
\sup _{\varepsilon>0}\left\|g^{\varepsilon}\right\|_{\left.L^{\infty}\left(\Omega^{\prime} \times[0, T] ; \mathbb{R}^{3}\right)\right)}<+\infty, \\
g^{\varepsilon} \rightarrow g \text { in } W^{1,1}\left([0, T] ; W^{1, p}\left(\Omega^{\prime} ; \mathbb{R}^{3}\right)\right), \quad \frac{1}{\varepsilon} \nabla_{3} g^{\varepsilon} \rightarrow H \text { in } W^{1,1}\left([0, T] ; L^{p}\left(\Omega^{\prime} ; \mathbb{R}^{3}\right)\right)
\end{array}\right.
$$

and that

$$
\sup _{\varepsilon>0}\left\|u^{\varepsilon}(t)\right\|_{L^{\infty}\left(\Omega^{\prime} ; \mathbb{R}^{3}\right)}<+\infty
$$

uniformly with respect to $t \in[0, T]$. Then, there exists a two-dimensional quasistatic evolution $[0, T] \ni$ $t \mapsto(u(t), \gamma(t))$ relative to the boundary data $g(t)$ for the relaxed model and a sequence $\left\{\varepsilon_{n}\right\} \searrow 0^{+}$such that for every $t \in[0, T]$, 


\section{JEAN-FranÇOIS BABADJIAN}

- $\Gamma^{\varepsilon_{n}}(t)$ converges to $\gamma(t)$ in the sense of Definition 4.1 and $u^{\varepsilon_{n}}(0) \rightarrow u(0)$ in $S B V^{p}\left(\Omega^{\prime} ; \mathbb{R}^{3}\right)$;

- $u^{\varepsilon_{n t}}(t) \rightarrow u(t)$ in $S B V^{p}\left(\Omega^{\prime} ; \mathbb{R}^{3}\right)$, for some $t$-dependent subsequence $\left\{\varepsilon_{n_{t}}\right\} \subset\left\{\varepsilon_{n}\right\}$;

- the total energy $\mathcal{E}_{\varepsilon_{n}}(t)$ converges to $\mathcal{E}(t)$ and more precisely,

$$
\left\{\begin{array}{l}
\int_{\Omega} W\left(\nabla_{\alpha} u^{\varepsilon_{n}}(t) \mid \frac{1}{\varepsilon_{n}} \nabla_{3} u^{\varepsilon_{n}}(t)\right) d x \rightarrow 2 \int_{\omega} \mathcal{Q} W_{0}\left(\nabla_{\alpha} u(t)\right) d x_{\alpha}, \\
\int_{\Gamma^{\varepsilon_{n}}(t)}\left|\left(\left(\nu_{\Gamma^{\varepsilon_{n}}(t)}\right)_{\alpha} \mid \frac{1}{\varepsilon_{n}}\left(\nu_{\Gamma^{\varepsilon_{n}}(t)}\right)_{3}\right)\right| d \mathcal{H}^{2} \rightarrow 2 \mathcal{H}^{1}(\gamma(t)) .
\end{array}\right.
$$

As it has been discussed in the introduction, it seems that the right functional setting for this kind of problems is that of Generalized Special Functions of Bounded Variation (see [13]) adding appropriate conservative surface and body forces. Here, as in [14], we will only deal with Special Functions of Bounded Variation, imposing, without any justification, that the minimizing fields are bounded in $L^{\infty}\left(\Omega ; \mathbb{R}^{3}\right)$, uniformly in time (see (2.8) and the proof of Lemma 5.1). Note that this assumption is automatically satisfied in the scalar case by a truncation argument, provided that $g^{\varepsilon}$ is also bounded in $L^{\infty}\left(\Omega^{\prime} \times[0, T] ; \mathbb{R}\right)$. This will let us get a bound in $B V\left(\Omega ; \mathbb{R}^{3}\right)$ for any sequence with bounded energy and thus apply Ambrosio's Compactness Theorem in $S B V$ (Theorem 4.8 in [2]). In a $G S B V$ context, we could state a result similar to that in [13] upon adding some appropriate conservative body and surface loadings with the right order of magnitude. Adequate coerciveness assumptions would allow us to get rid of the empirical $L^{\infty}$-bound (2.8) because we would have natural compactness in a suitable subspace of $G S B V\left(\Omega ; \mathbb{R}^{3}\right)$ and we would then obtain a membrane limit model.

Let us first state a compactness result in $S B V^{p}\left(\Omega ; \mathbb{R}^{3}\right)$ which ensures that any limit deformation field does not depend on the $x_{3}$ variable.

Lemma 2.2. Let $\left\{\varepsilon_{n}\right\} \searrow 0^{+}$and $\left\{u_{n}\right\} \subset S B V^{p}\left(\Omega ; \mathbb{R}^{3}\right)$ such that

$$
\sup _{n \in \mathbb{N}}\left\{\left\|u_{n}\right\|_{L^{\infty}\left(\Omega ; \mathbb{R}^{3}\right)}+\int_{\Omega}\left|\left(\nabla_{\alpha} u_{n} \mid \frac{1}{\varepsilon_{n}} \nabla_{3} u_{n}\right)\right|^{p} d x+\int_{S\left(u_{n}\right)}\left|\left(\left(\nu_{u_{n}}\right)_{\alpha} \mid \frac{1}{\varepsilon_{n}}\left(\nu_{u_{n}}\right)_{3}\right)\right| d \mathcal{H}^{2}\right\}<+\infty .
$$

Then, there exists a subsequence $\left\{\varepsilon_{n_{k}}\right\} \subset\left\{\varepsilon_{n}\right\}$ and a function $u \in S B V^{p}\left(\omega ; \mathbb{R}^{3}\right)$ such that $u_{n_{k}} \rightarrow u$ in $S B V^{p}\left(\Omega ; \mathbb{R}^{3}\right)$.

Proof. In view of (2.9), we have in particular

$$
\sup _{n \in \mathbb{N}}\left\{\left\|u_{n}\right\|_{L^{\infty}\left(\Omega ; \mathbb{R}^{3}\right)}+\left\|\nabla u_{n}\right\|_{L^{p}\left(\Omega ; \mathbb{R}^{3 \times 3}\right)}+\mathcal{H}^{2}\left(S\left(u_{n}\right)\right)\right\}<+\infty .
$$

Thus, according to Ambrosio's Compactness Theorem (Theorem 4.8 in [2]), we can find a subsequence $\left\{\varepsilon_{n_{k}}\right\} \subset\left\{\varepsilon_{n}\right\}$ and a function $u \in S B V^{p}\left(\Omega ; \mathbb{R}^{3}\right)$ such that $u_{n_{k}} \rightarrow u$ in $S B V^{p}\left(\Omega ; \mathbb{R}^{3}\right)$. Let us show that $u$ does not depend on $x_{3}$. Indeed, (2.9) implies that

$$
\frac{1}{\varepsilon_{n_{k}}}\left[\int_{\Omega}\left|\nabla_{3} u_{n_{k}}\right|^{p} d x+\int_{S\left(u_{n_{k}}\right)}\left|\left(\nu_{u_{n_{k}}}\right)_{3}\right| d \mathcal{H}^{2}\right] \leq C .
$$

By lower semicontinuity of

$$
v \mapsto \int_{\Omega}\left|\nabla_{3} v\right|^{p} d x+\int_{S(v)}\left|\left(\nu_{v}\right)_{3}\right| d \mathcal{H}^{2}
$$


with respect to the $S B V^{p}\left(\Omega ; \mathbb{R}^{3}\right)$-convergence (see $\left.[6,9]\right)$, we deduce that $\nabla_{3} u=0 \mathcal{L}^{3}$-a.e. in $\Omega$ and that $\left(\nu_{u}\right)_{3}=0 \mathcal{H}^{2}$-a.e. in $S(u)$. Since $u \in S B V^{p}\left(\Omega ; \mathbb{R}^{3}\right)$, it implies that $D_{3} u=0$ in the sense of Radon measures and thus $u \in S B V^{p}\left(\omega ; \mathbb{R}^{3}\right)$.

Note that the $L^{\infty}$-bound in (2.9) will follow from assumption (2.8), an assumption that we do not attempt to justify, while the two other bounds will appear naturally in the energy estimates.

\section{A $\Gamma$-convergence result}

This section is devoted to the study of the static problem. Let us define $\mathcal{I}_{\varepsilon}: B V\left(\Omega ; \mathbb{R}^{3}\right) \rightarrow \overline{\mathbb{R}}$ by

$$
\mathcal{I}_{\varepsilon}(u):=\left\{\begin{array}{cc}
\int_{\Omega} W\left(\nabla_{\alpha} u \mid \frac{1}{\varepsilon} \nabla_{3} u\right) d x+\int_{S(u)}\left|\left(\left(\nu_{u}\right)_{\alpha} \mid \frac{1}{\varepsilon}\left(\nu_{u}\right)_{3}\right)\right| d \mathcal{H}^{2} & \text { if } u \in S B V^{p}\left(\Omega ; \mathbb{R}^{3}\right), \\
+\infty & \text { otherwise }
\end{array}\right.
$$

Then, the following $\Gamma$-convergence result holds:

Theorem 3.1. Let $\omega$ be a bounded open subset of $\mathbb{R}^{2}$ and $W: \mathbb{R}^{3 \times 3} \rightarrow \mathbb{R}$ be a continuous function satisfying (2.1). Then the functional $\mathcal{I}_{\varepsilon} \Gamma$-converges for the strong $L^{1}\left(\Omega ; \mathbb{R}^{3}\right)$-topology towards $\mathcal{J}: B V\left(\Omega ; \mathbb{R}^{3}\right) \rightarrow \overline{\mathbb{R}}$ defined by

$$
\mathcal{J}(u):=\left\{\begin{array}{cl}
2 \int_{\omega} \mathcal{Q} W_{0}\left(\nabla_{\alpha} u\right) d x_{\alpha}+2 \mathcal{H}^{1}(S(u)) & \text { if } u \in S B V^{p}\left(\omega ; \mathbb{R}^{3}\right), \\
+\infty & \text { otherwise. }
\end{array}\right.
$$

Remark 3.2. It has been noted in [22] p. 556 (see also [9] p. 306) that the function $\mathbb{R}^{3 \times 3} \ni\left(\bar{\xi} \mid \xi_{3}\right) \mapsto$ $\mathcal{Q} W_{0}(\bar{\xi})$ is quasiconvex: for any $\varphi \in W_{0}^{1, \infty}\left(Q ; \mathbb{R}^{3}\right)$,

$$
\mathcal{Q} W_{0}(\bar{\xi}) \leq \int_{Q} \mathcal{Q} W_{0}\left(\bar{\xi}+\nabla_{\alpha} \varphi(y)\right) d y
$$

where $Q:=(0,1)^{3}$ is the unit square of $\mathbb{R}^{3}$.

We first localize our functionals on $\mathcal{A}(\omega)$, the family of open subsets of $\omega$. Define $\mathcal{I}_{\varepsilon}$ and $\mathcal{J}$ : $B V\left(\Omega ; \mathbb{R}^{3}\right) \times \mathcal{A}(\omega) \rightarrow \overline{\mathbb{R}}$ by

$$
\mathcal{I}_{\varepsilon}(u ; A):=\left\{\begin{array}{cl}
\int_{A \times I} W\left(\nabla_{\alpha} u \mid \frac{1}{\varepsilon} \nabla_{3} u\right) d x & \text { if } u \in S B V^{p}\left(A \times I ; \mathbb{R}^{3}\right), \\
+\int_{S(u) \cap[A \times I]}\left|\left(\left(\nu_{u}\right)_{\alpha} \mid \frac{1}{\varepsilon}\left(\nu_{u}\right)_{3}\right)\right| d \mathcal{H}^{2} & \\
+\infty & \text { otherwise }
\end{array}\right.
$$

and

$$
\mathcal{J}(u ; A):=\left\{\begin{array}{cl}
2 \int_{A} \mathcal{Q} W_{0}\left(\nabla_{\alpha} u\right) d x_{\alpha}+2 \mathcal{H}^{1}(S(u) \cap A) & \text { if } u \in S B V^{p}\left(A ; \mathbb{R}^{3}\right) \\
+\infty & \text { otherwise }
\end{array}\right.
$$


JEAN-FranÇOIS BABADJian

For every sequence $\left\{\varepsilon_{j}\right\} \searrow 0^{+}$and all $(u ; A) \in B V\left(\Omega ; \mathbb{R}^{3}\right) \times \mathcal{A}(\omega)$, we define the $\Gamma$-lower limit by

$$
\mathcal{I}(u ; A):=\inf _{\left\{u_{j}\right\}}\left\{\liminf _{j \rightarrow+\infty} \mathcal{I}_{\varepsilon_{j}}\left(u_{j} ; A\right): u_{j} \rightarrow u \text { in } L^{1}\left(A \times I ; \mathbb{R}^{3}\right)\right\} .
$$

Let $\mathcal{R}(\omega)$ be the countable subfamily of $\mathcal{A}(\omega)$ obtained by taking every finite union of open cubes of $\omega$, centered at rational points and with rational edge length. Theorem 8.5 in [12] together with a diagonalization argument imply the existence of a subsequence $\left\{\varepsilon_{n}\right\} \equiv\left\{\varepsilon_{j_{n}}\right\}$ such that, for any $C \in \mathcal{R}(\omega)$ (or $C=\omega), \mathcal{I}(\cdot ; C)$ is the $\Gamma$-limit of $\mathcal{I}_{\varepsilon_{n}}(\cdot ; C)$ for the strong $L^{1}\left(C \times I ; \mathbb{R}^{3}\right)$-topology. To prove Theorem 3.1 , it is enough to show that $\mathcal{I}(u ; \omega)=\mathcal{J}(u ; \omega)$.

\subsection{A truncation argument}

The main problem with definition (3.3) of $\mathcal{I}$ is that any minimizing sequence is not necessarily bounded in $B V\left(\Omega ; \mathbb{R}^{3}\right)$ and thus, not necessarily weakly convergent in this space. What is missing is either a bound on the jump part of the derivative (this bound was appearing naturally in $[9,6]$ because the authors were considering a surface energy density with linear growth with respect to the jump of the deformation), or an $L^{\infty}$-bound on the minimizing sequences. In the spirit of [17], we define for all $(u ; A) \in B V\left(\Omega ; \mathbb{R}^{3}\right) \times \mathcal{A}(\omega)$

$$
\mathcal{I}_{\infty}(u ; A):=\inf _{\left\{u_{n}\right\}}\left\{\liminf _{n \rightarrow+\infty} \mathcal{I}_{\varepsilon_{n}}\left(u_{n} ; A\right): u_{n} \rightarrow u \text { in } L^{1}\left(A \times I ; \mathbb{R}^{3}\right), \sup _{n \in \mathbb{N}}\left\|u_{n}\right\|_{L^{\infty}\left(A \times I ; \mathbb{R}^{3}\right)}<+\infty\right\} .
$$

Obviously, we have that $\mathcal{I}(u ; A) \leq \mathcal{I}_{\infty}(u ; A)$. In fact, we will prove that both functionals coincide if the deformation belongs to $B V\left(\Omega ; \mathbb{R}^{3}\right) \cap L^{\infty}\left(\Omega ; \mathbb{R}^{3}\right)$ in Lemma 3.3 below. Thus for a deformation $u \in B V\left(\Omega ; \mathbb{R}^{3}\right) \cap L^{\infty}\left(\Omega ; \mathbb{R}^{3}\right)$, strong $L^{1}\left(\Omega ; \mathbb{R}^{3}\right)$-convergence and weak $B V\left(\Omega ; \mathbb{R}^{3}\right)$-convergence are, in a sense, equivalent for the computation of the $\Gamma$-limit. This will permit us to prove in Lemma 3.6 that for such deformations $\mathcal{I}_{\infty}(u ; \cdot)$ is a Radon measure on $\omega$, absolutely continuous with respect to sum of the Lebesgue measure and of the restriction of the Hausdorff measure to $S(u)$. By Lebesgue's Decomposition Theorem, it will be enough to identify the Radon-Nikodym derivatives of $\mathcal{I}_{\infty}(u ; \cdot)$ with respect to $\mathcal{L}^{2}$ and $\mathcal{H}_{[S(u)}^{1}$. This will be done for the proof of the upper bound in Lemma 3.7 by a blow up argument. The general case will be treated in Lemma 3.8 thanks to a truncation argument. The lower bound will be proved in Lemma 3.9 using the quasiconvexity properties of $\mathcal{Q} W_{0}$ (see Remark 3.2) and a lower semicontinuity result in $S B V^{p}\left(\Omega ; \mathbb{R}^{3}\right)$.

Lemma 3.3. For any $C \in \mathcal{R}(\omega)$ (or $C=\omega$ ) and all $u \in B V\left(\Omega ; \mathbb{R}^{3}\right) \cap L^{\infty}\left(\Omega ; \mathbb{R}^{3}\right), \mathcal{I}(u ; C)=\mathcal{I}_{\infty}(u ; C)$.

Proof. It is enough to show that $\mathcal{I}(u ; C) \geq \mathcal{I}_{\infty}(u ; C)$ for all $u \in B V\left(\Omega ; \mathbb{R}^{3}\right) \cap L^{\infty}\left(\Omega ; \mathbb{R}^{3}\right)$. If $\mathcal{I}(u ; C)=+\infty$, the result is obvious, thus there is no loss of generality in assuming that $\mathcal{I}(u ; C)<+\infty$. By the very definition of the $\Gamma$-limit, there exists a sequence $u_{n} \rightarrow u$ in $L^{1}\left(C \times I ; \mathbb{R}^{3}\right)$ such that

$$
\mathcal{I}(u ; C)=\lim _{n \rightarrow+\infty} \mathcal{I}_{\varepsilon_{n}}\left(u_{n} ; C\right) .
$$

Since $\mathcal{I}(u ; C)<+\infty$, in view of (3.1), we deduce that, for $n$ large enough, $u_{n} \in S B V^{p}\left(C \times I ; \mathbb{R}^{3}\right)$. We consider only those $n$ 's.

Let us define a smooth truncation function $\varphi_{i} \in \mathcal{C}_{c}^{1}\left(\mathbb{R}^{3} ; \mathbb{R}^{3}\right)$ satisfying

$$
\varphi_{i}(z)=\left\{\begin{array}{cc}
z & \text { if } \quad|z|<e^{i} \\
0 & \text { if } \quad|z| \geq e^{i+1}
\end{array} \quad \text { and } \quad\left|\nabla \varphi_{i}(z)\right| \leq 1 .\right.
$$

Let $w_{n}^{i}:=\varphi_{i}\left(u_{n}\right)$, thanks to the Chain Rule formula, Theorem 3.96 in [2], $w_{n}^{i} \in S B V^{p}\left(C \times I ; \mathbb{R}^{3}\right)$ and

$$
\left\{\begin{array}{l}
\left\|w_{n}^{i}\right\|_{L^{\infty}\left(C \times I ; \mathbb{R}^{3}\right)} \leq e^{i}, \\
S\left(w_{n}^{i}\right) \subset S\left(u_{n}\right), \\
\nabla w_{n}^{i}(x)=\nabla \varphi_{i}\left(u_{n}(x)\right) \circ \nabla u_{n}(x) \quad \mathcal{L}^{3} \text {-a.e. on } C \times I .
\end{array}\right.
$$


QUASISTATIC EVOLUtion OF A BRITTLE THIN FILM

Since $u \in L^{\infty}\left(\Omega ; \mathbb{R}^{3}\right)$, we can choose $i$ large enough so that $u=\varphi_{i}(u)$, thus according to (3.5)

$$
\left\|w_{n}^{i}-u\right\|_{L^{1}\left(C \times I ; \mathbb{R}^{3}\right)}=\left\|\varphi_{i}\left(u_{n}\right)-\varphi_{i}(u)\right\|_{L^{1}\left(C \times I ; \mathbb{R}^{3}\right)} \leq\left\|u_{n}-u\right\|_{L^{1}\left(C \times I ; \mathbb{R}^{3}\right)} .
$$

The growth condition (2.1), (3.5) and (3.6) imply that

$$
\begin{aligned}
& \int_{C \times I} W\left(\nabla_{\alpha} w_{n}^{i} \mid \frac{1}{\varepsilon_{n}} \nabla_{3} w_{n}^{i}\right) d x \\
&=\int_{\left\{u_{n}<e^{i}\right\}} W\left(\nabla_{\alpha} u_{n} \mid \frac{1}{\varepsilon_{n}} \nabla_{3} u_{n}\right) d x \\
& \quad+\int_{\left\{e^{i} \leq u_{n}<e^{i+1}\right\}} W\left(\nabla \varphi_{i}\left(u_{n}\right) \circ \nabla_{\alpha} u_{n} \mid \frac{1}{\varepsilon_{n}} \nabla \varphi_{i}\left(u_{n}\right) \circ \nabla_{3} u_{n}\right) d x \\
& \quad+W(0) \mathcal{L}^{3}\left(\left\{u_{n} \geq e^{i+1}\right\}\right) \\
& \leq \int_{C \times I} W\left(\nabla_{\alpha} u_{n} \mid \frac{1}{\varepsilon_{n}} \nabla_{3} u_{n}\right) d x+\beta \mathcal{L}^{3}\left(\left\{u_{n} \geq e^{i}\right\}\right) \\
&+\beta \int_{\left\{e^{i} \leq u_{n}<e^{i+1}\right\}}\left|\left(\nabla_{\alpha} u_{n} \mid \frac{1}{\varepsilon_{n}} \nabla_{3} u_{n}\right)\right|^{p} d x \\
& \leq \int_{C \times I} W\left(\nabla_{\alpha} u_{n} \mid \frac{1}{\varepsilon_{n}} \nabla_{3} u_{n}\right) d x+\frac{\beta}{e^{i}}\left\|u_{n}\right\|_{L^{1}\left(C \times I ; \mathbb{R}^{3}\right)} \\
&+\beta \int_{\left\{e^{i} \leq u_{n}<e^{i+1}\right\}}\left|\left(\nabla_{\alpha} u_{n} \mid \frac{1}{\varepsilon_{n}} \nabla_{3} u_{n}\right)\right|^{p} d x
\end{aligned}
$$

where we have used Chebyshev's inequality. Let $M \in \mathbb{N}$, a summation for $i=1$ to $M$ implies using (3.6) and the fact that $\nu_{w_{n}^{i}}(x)= \pm \nu_{u_{n}}(x)$ for $\mathcal{H}^{2}$-a.e. $x \in S\left(w_{n}^{i}\right)$,

$$
\begin{gathered}
\frac{1}{M} \sum_{i=1}^{M}\left[\int_{C \times I} W\left(\nabla_{\alpha} w_{n}^{i} \mid \frac{1}{\varepsilon_{n}} \nabla_{3} w_{n}^{i}\right) d x+\int_{S\left(w_{n}^{i}\right) \cap[C \times I]}\left|\left(\left(\nu_{w_{n}^{i}}\right)_{\alpha} \mid \frac{1}{\varepsilon_{n}}\left(\nu_{w_{n}^{i}}\right)_{3}\right)\right| d \mathcal{H}^{2}\right] \\
\quad \leq \int_{C \times I} W\left(\nabla_{\alpha} u_{n} \mid \frac{1}{\varepsilon_{n}} \nabla_{3} u_{n}\right) d x+\int_{S\left(u_{n}\right) \cap[C \times I]}\left|\left(\left(\nu_{u_{n}}\right)_{\alpha} \mid \frac{1}{\varepsilon_{n}}\left(\nu_{u_{n}}\right)_{3}\right)\right| d \mathcal{H}^{2}+\frac{c}{M},
\end{gathered}
$$

where

$$
c=\beta \sup _{n \in \mathbb{N}}\left\|u_{n}\right\|_{L^{1}\left(C \times I ; \mathbb{R}^{3}\right)} \sum_{i \geq 1} \frac{1}{e^{i}}+\beta \sup _{n \in \mathbb{N}}\left\|\left(\nabla_{\alpha} u_{n} \mid \frac{1}{\varepsilon_{n}} \nabla_{3} u_{n}\right)\right\|_{L^{p}\left(C \times I ; \mathbb{R}^{3 \times 3}\right)}^{p}<+\infty .
$$

We may find some $i_{n} \in\{1, \ldots, M\}$ such that

$$
\begin{aligned}
& \int_{C \times I} W\left(\nabla_{\alpha} w_{n}^{i_{n}} \mid \frac{1}{\varepsilon_{n}} \nabla_{3} w_{n}^{i_{n}}\right) d x+\int_{S\left(w_{n}^{i_{n}}\right) \cap[C \times I]}\left|\left(\left(\nu_{w_{n}^{i_{n}}}\right)_{\alpha} \mid \frac{1}{\varepsilon_{n}}\left(\nu_{w_{n}^{i_{n}}}\right)_{3}\right)\right| d \mathcal{H}^{2} \\
& \leq \int_{C \times I} W\left(\nabla_{\alpha} u_{n} \mid \frac{1}{\varepsilon_{n}} \nabla_{3} u_{n}\right) d x+\int_{S\left(u_{n}\right) \cap[C \times I]}\left|\left(\left(\nu_{u_{n}}\right)_{\alpha} \mid \frac{1}{\varepsilon_{n}}\left(\nu_{u_{n}}\right)_{3}\right)\right| d \mathcal{H}^{2}+\frac{c}{M} .
\end{aligned}
$$

Set $v_{n}:=w_{n}^{i_{n}}$, thus in view of $(3.7), v_{n} \rightarrow u$ in $L^{1}\left(C \times I ; \mathbb{R}^{3}\right)$. Moreover, (3.6) implies that

$$
\left\|v_{n}\right\|_{L^{\infty}\left(C \times I ; \mathbb{R}^{3}\right)} \leq e^{i_{n}} \leq e^{M} .
$$

Finally, by virtue of (3.4) and (3.8),

$$
\mathcal{I}(u ; C)+\frac{c}{M} \geq \liminf _{n \rightarrow+\infty} \mathcal{I}_{\varepsilon_{n}}\left(v_{n} ; C\right) \geq \mathcal{I}_{\infty}(u ; C) .
$$




\section{JEAN-FranÇOIS BABADJIAN}

The proof is achieved upon letting $M$ tend to $+\infty$

To prove the upper bound in Lemma 3.7 below, we need a little bit more than the only continuity condition imposed on $W$, namely a $p$-Lipschitz condition. If $W$ was quasiconvex, this property would be immediate. Since we do not want to restrict too much the stored energy density, we will show that there is no loss of generality in assuming $W$ to be quasiconvex. Let $\mathcal{Q} W$ be the $3 \mathrm{D}$-quasiconvexification of $W$ defined by

$$
\mathcal{Q} W(\xi):=\inf _{\varphi \in W_{0}^{1, \infty}\left(Q ; \mathbb{R}^{3}\right)} \int_{Q} W(\xi+\nabla \varphi(x)) d x \quad \text { for all } \xi \in \mathbb{R}^{3 \times 3} .
$$

Lemma 3.4. For all $u \in S B V^{p}\left(\omega ; \mathbb{R}^{3}\right) \cap L^{\infty}\left(\omega ; \mathbb{R}^{3}\right)$, the value of $\mathcal{I}_{\infty}(u ; \omega)$ does not change if we replace $W$ by $\mathcal{Q} W$ in (3.1).

Proof. We denote $\mathcal{I}_{\varepsilon}^{\mathcal{Q}}\left(\right.$ resp. $\left.\mathcal{I}^{\mathcal{Q}}, \mathcal{I}_{\infty}^{\mathcal{Q}}\right)$, the value of $\mathcal{I}_{\varepsilon}$ (resp. $\left.\mathcal{I}, \mathcal{I}_{\infty}\right)$ with $\mathcal{Q} W$ instead of $W$ in (3.1). By the same arguments as above, we may assume that $\left\{\varepsilon_{n}\right\}$ is a subsequence of $\left\{\varepsilon_{j}\right\}$ such that $\mathcal{I}^{\mathcal{Q}}(u ; \omega)=\mathcal{I}_{\infty}^{\mathcal{Q}}(u ; \omega)$ is the $\Gamma$-limit of $\mathcal{I}_{\varepsilon_{n}}^{\mathcal{Q}}(u ; \omega)$, for every $u \in B V\left(\Omega ; \mathbb{R}^{3}\right) \cap L^{\infty}\left(\Omega ; \mathbb{R}^{3}\right)$.

Let $u \in S B V^{p}\left(\omega ; \mathbb{R}^{3}\right) \cap L^{\infty}\left(\omega ; \mathbb{R}^{3}\right)$, since $W \geq \mathcal{Q} W$, we obviously have $\mathcal{I}_{\infty}(u ; \omega) \geq \mathcal{I}_{\infty}^{\mathcal{Q}}(u ; \omega)$. Let us prove the converse inequality. By the definition of the $\Gamma$-limit, we may find a sequence $\left\{u_{n}\right\} \subset$ $S B V^{p}\left(\Omega ; \mathbb{R}^{3}\right)$ such that $u_{n} \rightarrow u$ in $L^{1}\left(\Omega ; \mathbb{R}^{3}\right), \sup _{n \in \mathbb{N}}\left\|u_{n}\right\|_{L^{\infty}\left(\Omega ; \mathbb{R}^{3}\right)}<+\infty$ and

$$
\mathcal{I}_{\infty}^{\mathcal{Q}}(u ; \omega)=\lim _{n \rightarrow+\infty}\left\{\int_{\Omega} \mathcal{Q} W\left(\nabla_{\alpha} u_{n} \mid \frac{1}{\varepsilon_{n}} \nabla_{3} u_{n}\right) d x+\int_{S\left(u_{n}\right)}\left|\left(\left(\nu_{u_{n}}\right)_{\alpha} \mid \frac{1}{\varepsilon_{n}}\left(\nu_{u_{n}}\right)_{3}\right)\right| d \mathcal{H}^{2}\right\} .
$$

We undo the scaling by letting $v_{n}\left(x_{\alpha}, x_{3}\right):=u_{n}\left(x_{\alpha}, x_{3} / \varepsilon_{n}\right)$. Then $v_{n} \in S B V^{p}\left(\Omega_{\varepsilon_{n}} ; \mathbb{R}^{3}\right)$,

$$
\frac{1}{\varepsilon_{n}} \int_{\Omega_{\varepsilon_{n}}}\left|v_{n}-u\right| d x \rightarrow 0, \quad \sup _{n \in \mathbb{N}}\left\|v_{n}\right\|_{L^{\infty}\left(\Omega_{\varepsilon_{n}} ; \mathbb{R}^{3}\right)}<+\infty
$$

and

$$
\mathcal{I}_{\infty}^{\mathcal{Q}}(u ; \omega)=\lim _{n \rightarrow+\infty} \frac{1}{\varepsilon_{n}}\left\{\int_{\Omega_{\varepsilon_{n}}} \mathcal{Q} W\left(\nabla v_{n}\right) d x+\mathcal{H}^{2}\left(S\left(v_{n}\right)\right)\right\} .
$$

For all $n \in \mathbb{N}$, Theorem 8.1 in [7] and Proposition 2.8 in [17] yield the existence of a sequence $\left\{v_{n, k}\right\}_{k \in \mathbb{N}} \subset$ $S B V^{p}\left(\Omega_{\varepsilon_{n}} ; \mathbb{R}^{3}\right)$ satisfying $v_{n, k} \rightarrow v_{n}$ in $L^{1}\left(\Omega_{\varepsilon_{n}} ; \mathbb{R}^{3}\right)$ as $k \rightarrow+\infty$,

$$
\int_{\Omega_{\varepsilon_{n}}} \mathcal{Q} W\left(\nabla v_{n}\right) d x+\mathcal{H}^{2}\left(S\left(v_{n}\right)\right)=\lim _{k \rightarrow+\infty}\left\{\int_{\Omega_{\varepsilon_{n}}} W\left(\nabla v_{n, k}\right) d x+\mathcal{H}^{2}\left(S\left(v_{n, k}\right)\right)\right\}
$$

and $\sup _{k \in \mathbb{N}}\left\|v_{n, k}\right\|_{L^{\infty}\left(\Omega_{\varepsilon_{n}} ; \mathbb{R}^{3}\right)}<+\infty$. Since the previous bound is of the form $e^{M}$ (see [17] p. 417), for some constant $M>0$ independent of $n$, this last relation holds uniformly with respect to $n \in \mathbb{N}$. Gathering (3.9) and (3.10), we get

$$
\mathcal{I}_{\infty}^{\mathcal{Q}}(u ; \omega)=\lim _{n \rightarrow+\infty} \lim _{k \rightarrow+\infty} \frac{1}{\varepsilon_{n}}\left\{\int_{\Omega_{\varepsilon_{n}}} W\left(\nabla v_{n, k}\right) d x+\mathcal{H}^{2}\left(S\left(v_{n, k}\right)\right)\right\} .
$$

Let $u_{n, k}\left(x_{\alpha}, x_{3}\right):=v_{n, k}\left(x_{\alpha}, \varepsilon_{n} x_{3}\right)$, then $\left\{u_{n, k}\right\} \subset S B V^{p}\left(\Omega ; \mathbb{R}^{3}\right)$,

$$
\lim _{n \rightarrow+\infty} \lim _{k \rightarrow+\infty} \int_{\Omega}\left|u_{n, k}-u\right| d x=0, \quad \sup _{n, k \in \mathbb{N}}\left\|u_{n, k}\right\|_{L^{\infty}\left(\Omega ; \mathbb{R}^{3}\right)}<+\infty
$$


and

$$
\begin{aligned}
\mathcal{I}_{\infty}^{\mathcal{Q}}(u ; \omega)=\lim _{n \rightarrow+\infty} \lim _{k \rightarrow+\infty}\left\{\int_{\Omega} W\left(\nabla_{\alpha} u_{n, k} \mid \frac{1}{\varepsilon_{n}} \nabla_{3} u_{n, k}\right) d x\right. & \\
& \left.+\int_{S\left(u_{n, k}\right)}\left|\left(\left(\nu_{u_{n, k}}\right)_{\alpha} \mid \frac{1}{\varepsilon_{n}}\left(\nu_{u_{n, k}}\right)_{3}\right)\right| d \mathcal{H}^{2}\right\} .
\end{aligned}
$$

By a diagonalization argument, we can find a sequence $k_{n} \nearrow+\infty$ such that upon denoting $w_{n}:=u_{n, k_{n}}$, then $w_{n} \in S B V^{p}\left(\Omega ; \mathbb{R}^{3}\right), w_{n} \rightarrow u$ in $L^{1}\left(\Omega ; \mathbb{R}^{3}\right), \sup _{n \in \mathbb{N}}\left\|w_{n}\right\|_{L^{\infty}\left(\Omega ; \mathbb{R}^{3}\right)}<+\infty$ and

$$
\begin{aligned}
\mathcal{I}_{\infty}^{\mathcal{Q}}(u ; \omega) & =\lim _{n \rightarrow+\infty}\left\{\int_{\Omega} W\left(\nabla_{\alpha} w_{n} \mid \frac{1}{\varepsilon_{n}} \nabla_{3} w_{n}\right) d x+\int_{S\left(w_{n}\right)}\left|\left(\left(\nu_{w_{n}}\right)_{\alpha} \mid \frac{1}{\varepsilon_{n}}\left(\nu_{w_{n}}\right)_{3}\right)\right| d \mathcal{H}^{2}\right\} \\
& \geq \mathcal{I}_{\infty}(u ; \omega) .
\end{aligned}
$$

Remark 3.5. From Comments on Theorem 8. (iii) p. 560 in [22], we always have $\mathcal{Q} W_{0}=\mathcal{Q}\left((\mathcal{Q} W)_{0}\right)$. As a consequence, by Lemma 3.4, we may assume without loss of generality, upon replacing $W$ by $\mathcal{Q} W$, that $W$ is quasiconvex. In particular (see [11]), the following $p$-Lipschitz condition holds,

$$
\left|W\left(\xi_{1}\right)-W\left(\xi_{2}\right)\right| \leq \beta\left(1+\left|\xi_{1}\right|^{p-1}+\left|\xi_{2}\right|^{p-1}\right)\left|\xi_{1}-\xi_{2}\right|, \text { for all } \xi_{1}, \xi_{2} \in \mathbb{R}^{3 \times 3} .
$$

\subsection{Integral Representation of the $\Gamma$-limit}

Lemma 3.3 is essential for the proof of the following result because it allows us to replace strong $L^{1}\left(\Omega ; \mathbb{R}^{3}\right)$ convergence of any minimizing sequence by strong $L^{p}\left(\Omega ; \mathbb{R}^{3}\right)$-convergence, where $1<p<\infty$ is the same exponent as in (2.1).

Lemma 3.6. For all $u \in S B V^{p}\left(\omega ; \mathbb{R}^{3}\right) \cap L^{\infty}\left(\omega ; \mathbb{R}^{3}\right), \mathcal{I}_{\infty}(u ; \cdot)$ is the restriction to $\mathcal{A}(\omega)$ of a Radon measure absolutely continuous with respect to $\mathcal{L}^{2}+\mathcal{H}_{[S(u)}^{1}$.

Proof. Let $u \in S B V^{p}\left(\omega ; \mathbb{R}^{3}\right) \cap L^{\infty}\left(\omega ; \mathbb{R}^{3}\right)$. The $p$-growth condition (2.1) implies that

$$
\mathcal{I}_{\infty}(u ; A) \leq 2 \beta \int_{A}\left(1+\left|\nabla_{\alpha} u\right|^{p}\right) d x_{\alpha}+2 \mathcal{H}^{1}(S(u) \cap A) .
$$

Thus, thanks to e.g. Lemma 7.3 in [10], it is enough to show the existence of a Radon measure $\hat{\mu}$ on $\mathbb{R}^{2}$ such that for every $A, B$ and $C \in \mathcal{A}(\omega)$,

(i) $\mathcal{I}_{\infty}(u ; A) \leq \mathcal{I}_{\infty}(u ; A \backslash \bar{C})+\mathcal{I}_{\infty}(u ; B)$ if $\bar{C} \subset B \subset A$;

(ii) for any $\delta>0$, there exists $C_{\delta} \in \mathcal{A}(\omega)$ such that $\bar{C}_{\delta} \subset A$ and $\mathcal{I}_{\infty}\left(u ; A \backslash \bar{C}_{\delta}\right) \leq \delta$;

(iii) $\mathcal{I}_{\infty}(u ; \omega) \geq \hat{\mu}\left(\mathbb{R}^{2}\right)$;

(iv) $\mathcal{I}_{\infty}(u ; A) \leq \hat{\mu}(\bar{A})$.

Since $u \in S B V^{p}\left(\omega ; \mathbb{R}^{3}\right), \mathcal{H}^{1}(S(u))<+\infty$, thus $\mathcal{H}_{[S(u)}^{1}$ is a Radon measure. Then, for any $\delta>0$, there exists $C_{\delta} \in \mathcal{A}(\omega)$ such that $\bar{C}_{\delta} \subset A$ and

$$
2 \beta \int_{A \backslash \bar{C}_{\delta}}\left(1+\left|\nabla_{\alpha} u\right|^{p}\right) d x_{\alpha}+2 \mathcal{H}^{1}\left(S(u) \cap\left[A \backslash \bar{C}_{\delta}\right]\right) \leq \delta .
$$




\section{JEAN-FranÇOIS BABADJIAN}

Thus, thanks to the growth condition (2.1), we have $\mathcal{I}_{\infty}\left(u ; A \backslash \bar{C}_{\delta}\right) \leq \delta$ and item (ii) holds true. Furthermore, by Lemma 3.3 and the definition of the $\Gamma$-limit, there exists a sequence $\left\{u_{n}\right\} \subset S B V^{p}\left(\Omega ; \mathbb{R}^{3}\right)$ such that $u_{n} \rightarrow u$ in $L^{1}\left(\Omega ; \mathbb{R}^{3}\right)$ and $\mathcal{I}_{\varepsilon_{n}}\left(u_{n} ; \omega\right) \rightarrow \mathcal{I}_{\infty}(u ; \omega)$. Denoting by

$$
\mu_{n}:=W\left(\nabla_{\alpha} u_{n} \mid \frac{1}{\varepsilon_{n}} \nabla_{3} u_{n}\right) \mathcal{L}_{\lfloor\Omega}^{3}+\left|\left(\left(\nu_{u_{n}}\right)_{\alpha} \mid \frac{1}{\varepsilon_{n}}\left(\nu_{u_{n}}\right)_{3}\right)\right| \mathcal{H}_{\left\lfloor S\left(u_{n}\right) \cap \Omega\right.}^{2}
$$

for a subsequence of $\left\{\varepsilon_{n}\right\}$ (not relabeled), there exists a Radon measure $\mu$ such that $\mu_{n} \stackrel{*}{\rightarrow} \mu$ in $\mathcal{M}_{b}\left(\mathbb{R}^{3}\right)$. Let $\hat{\mu}(B):=\mu(B \times[-1,1])$, for every Borel set $B \subset \mathbb{R}^{2}$. Thus, $\hat{\mu}\left(\mathbb{R}^{2}\right) \leq \mathcal{I}_{\infty}(u ; \omega)$ and item (iii) follows. Moreover, for every $A \in \mathcal{A}(\omega)$,

$$
\mathcal{I}_{\infty}(u ; A) \leq \liminf _{n \rightarrow+\infty} \mathcal{I}_{\varepsilon_{n}}\left(u_{n} ; A\right) \leq \limsup _{n \rightarrow+\infty} \mu_{n}(\bar{A} \times[-1,1]) \leq \mu(\bar{A} \times[-1,1])=\hat{\mu}(\bar{A})
$$

which establishes item (iv). We now show the subadditivity condition expressed in item (i). For any $\eta>0$, we can find a sequence $\left\{v_{n}\right\} \subset S B V^{p}\left([A \backslash \bar{C}] \times I ; \mathbb{R}^{3}\right)$ such that $v_{n} \rightarrow u$ in $L^{1}\left([A \backslash \bar{C}] \times I ; \mathbb{R}^{3}\right)$,

$$
\sup _{n \in \mathbb{N}}\left\|v_{n}\right\|_{L^{\infty}\left([A \backslash \bar{C}] \times I ; \mathbb{R}^{3}\right)}<+\infty
$$

and

$$
\liminf _{n \rightarrow+\infty} \mathcal{I}_{\varepsilon_{n}}\left(v_{n} ; A \backslash \bar{C}\right) \leq \mathcal{I}_{\infty}(u ; A \backslash \bar{C})+\eta .
$$

In particular, $v_{n} \rightarrow u$ in $L^{p}\left([A \backslash \bar{C}] \times I ; \mathbb{R}^{3}\right)$ and we may extract a subsequence $\left\{\varepsilon_{n_{k}}\right\} \subset\left\{\varepsilon_{n}\right\}$ for which

$$
\lim _{k \rightarrow+\infty} \mathcal{I}_{\varepsilon_{n_{k}}}\left(v_{n_{k}} ; A \backslash \bar{C}\right) \leq \mathcal{I}_{\infty}(u ; A \backslash \bar{C})+\eta
$$

Let $R_{0} \in \mathcal{R}(\omega)$ satisfy $C \subset \subset R_{0} \subset \subset B$, thus, since $\mathcal{I}\left(u ; R_{0}\right)$ is the $\Gamma$-limit of $\mathcal{I}_{\varepsilon_{n_{k}}}\left(u ; R_{0}\right)$, thanks to Lemma 3.3, there exists a sequence $\left\{u_{k}\right\} \subset S B V^{p}\left(R_{0} \times I ; \mathbb{R}^{3}\right)$ such that $u_{k} \rightarrow u$ in $L^{1}\left(R_{0} \times I ; \mathbb{R}^{3}\right)$,

$$
\sup _{k \in \mathbb{N}}\left\|u_{k}\right\|_{L^{\infty}\left(R_{0} \times I ; \mathbb{R}^{3}\right)}<+\infty
$$

and

$$
\mathcal{I}_{\varepsilon_{n_{k}}}\left(u_{k} ; R_{0}\right) \rightarrow \mathcal{I}_{\infty}\left(u ; R_{0}\right) .
$$

In particular, we have $u_{k} \rightarrow u$ in $L^{p}\left(R_{0} \times I ; \mathbb{R}^{3}\right)$. According to the $p$-coercivity condition (2.1) the following sequence of Radon measures

$$
\begin{aligned}
\lambda_{k}:=(1 & \left.+\left|\left(\nabla_{\alpha} v_{n_{k}} \mid \frac{1}{\varepsilon_{n_{k}}} \nabla_{3} v_{n_{k}}\right)\right|^{p}\right) \mathcal{L}_{\left\lfloor\left(R_{0} \backslash \bar{C}\right) \times I\right.}^{3}+\left|\left(\left(\nu_{v_{n_{k}}}\right)_{\alpha} \mid \frac{1}{\varepsilon_{n_{k}}}\left(\nu_{v_{n_{k}}}\right)_{3}\right)\right| \mathcal{H}_{\left\lfloor S\left(v_{n_{k}}\right) \cap\left[\left(R_{0} \backslash \bar{C}\right) \times I\right]\right.}^{2} \\
& +\left(1+\left|\left(\nabla_{\alpha} u_{k} \mid \frac{1}{\varepsilon_{n_{k}}} \nabla_{3} u_{k}\right)\right|^{p}\right) \mathcal{L}_{\left\lfloor\left(R_{0} \backslash \bar{C}\right) \times I\right.}^{3}+\left|\left(\left(\nu_{u_{k}}\right)_{\alpha} \mid \frac{1}{\varepsilon_{n_{k}}}\left(\nu_{u_{k}}\right)_{3}\right)\right| \mathcal{H}_{\left\lfloor S\left(u_{k}\right) \cap\left[\left(R_{0} \backslash \bar{C}\right) \times I\right]\right.}^{2}
\end{aligned}
$$

is uniformly bounded, and thus, for a subsequence that will not be relabeled, there exists a positive Radon measure $\lambda$ such that $\lambda_{k} \stackrel{*}{\rightarrow} \lambda$ in $\mathcal{M}_{b}\left(R_{0} \backslash \bar{C}\right)$.

Let $t>0$, define $R_{t}:=\left\{x_{\alpha} \in R_{0}: \operatorname{dist}\left(x_{\alpha}, \partial R_{0}\right)>t\right\}$ and for any $0<\delta<\eta, L_{\delta}:=R_{\eta-\delta} \backslash \bar{R}_{\eta+\delta}$. Since we are localizing in $\mathbb{R}^{2}$, we consider a cut-off function $\varphi_{\delta} \in \mathcal{C}_{c}^{\infty}\left(R_{\eta-\delta} ;[0,1]\right)$ depending only on $x_{\alpha}$ and satisfying $\varphi_{\delta}=1$ on $R_{\eta}$ and $\left\|\varphi_{\delta}\right\|_{L^{\infty}\left(R_{\eta-\delta}\right)} \leq C / \delta$. Define

$$
w_{k}(x):=u_{k}(x) \varphi_{\delta}\left(x_{\alpha}\right)+v_{n_{k}}(x)\left(1-\varphi_{\delta}\left(x_{\alpha}\right)\right) .
$$


Then, $w_{k} \in S B V^{p}\left(A \times I ; \mathbb{R}^{3}\right), w_{k} \rightarrow u$ in $L^{p}\left(A \times I ; \mathbb{R}^{3}\right)$ and in view of (3.13) and (3.15)

$$
\sup _{k \in \mathbb{N}}\left\|w_{k}\right\|_{L^{\infty}\left(A \times I ; \mathbb{R}^{3}\right)} \leq \sup _{k \in \mathbb{N}}\left\|u_{k}\right\|_{L^{\infty}\left(R_{0} \times I ; \mathbb{R}^{3}\right)}+\sup _{k \in \mathbb{N}}\left\|v_{n_{k}}\right\|_{L^{\infty}\left([A \backslash \bar{C}] \times I ; \mathbb{R}^{3}\right)}<+\infty .
$$

From (3.3), (3.14) and (3.16), we deduce that

$$
\begin{aligned}
\mathcal{I}_{\infty}(u ; A) \leq & \liminf _{k \rightarrow+\infty} \mathcal{I}_{\varepsilon_{n_{k}}}\left(w_{k} ; A\right) \\
\leq & \liminf _{k \rightarrow+\infty}\left\{\mathcal{I}_{\varepsilon_{n_{k}}}\left(u_{k} ; R_{\eta+\delta}\right)+\mathcal{I}_{\varepsilon_{n_{k}}}\left(v_{n_{k}} ; A \backslash \bar{R}_{\eta-\delta}\right)\right. \\
& \left.\quad+C \lambda_{k}\left(L_{\delta}\right)+\frac{C}{\delta^{p}} \int_{L_{\delta} \times I}\left|u_{k}-v_{n_{k}}\right|^{p} d x .\right\} \\
& \leq \mathcal{I}_{\infty}\left(u ; R_{0}\right)+\mathcal{I}_{\infty}(u ; A \backslash \bar{C})+\eta+C \lambda\left(\bar{L}_{\delta}\right) \\
\leq & \mathcal{I}_{\infty}(u ; B)+\mathcal{I}_{\infty}(u ; A \backslash \bar{C})+\eta+C \lambda\left(\bar{L}_{\delta}\right),
\end{aligned}
$$

where we have used the fact that $\mathcal{I}_{\infty}(u ; \cdot)$ is an increasing set function. Note that the previous computation would not hold if we had considered $\mathcal{I}$ instead of $\mathcal{I}_{\infty}$ because the minimizing sequences would only converge in $L^{1}$. Letting $\delta$ tend to zero, we obtain,

$$
\mathcal{I}_{\infty}(u ; A) \leq \mathcal{I}_{\infty}(u ; B)+\mathcal{I}_{\infty}(u ; A \backslash \bar{C})+\eta+C \lambda\left(\partial R_{\eta}\right) .
$$

Now choose a sequence $\eta_{h} \rightarrow 0$ such that $\lambda\left(\partial R_{\eta_{h}}\right)=0$. Letting $h \nearrow+\infty$ yields

$$
\mathcal{I}_{\infty}(u ; A) \leq \mathcal{I}_{\infty}(u ; B)+\mathcal{I}_{\infty}(u ; A \backslash \bar{C})
$$

which completes the proof of item (i). Thus, according to $(3.12)$ and Lemma 7.3 in $[10], \mathcal{I}_{\infty}(u ; \cdot)$ is the restriction to $\mathcal{A}(\omega)$ of the Radon measure $\hat{\mu}$ which is absolutely continuous with respect to $\mathcal{L}^{2}+\mathcal{H}_{[S(u)}^{1}$.

As a consequence of Lemma 3.6 and Lebesgue's Decomposition Theorem, there exists a $\mathcal{L}^{2}$-measurable function $h$ and a $\mathcal{H}_{\lfloor S(u)}^{1}$-measurable function $g$ such that for every $A \in \mathcal{A}(\omega)$,

$$
\mathcal{I}_{\infty}(u ; A)=\int_{A} h d \mathcal{L}^{2}+\int_{A \cap S(u)} g d \mathcal{H}^{1}
$$

We denote by $Q^{\prime}\left(x_{0}, \rho\right)$ the open cube of $\mathbb{R}^{2}$ centered at $x_{0} \in \omega$ and of side length $\rho>0$, where $\rho$ is small enough so that $Q^{\prime}\left(x_{0}, \rho\right) \in \mathcal{A}(\omega)$. Since the measures $\mathcal{L}^{2}$ and $\mathcal{H}_{[S(u)}^{1}$ are mutually singular, $h$ is the Radon-Nikodym derivative of $\mathcal{I}_{\infty}(u ; \cdot)$ with respect to $\mathcal{L}^{2}$,

$$
h\left(x_{0}\right)=\lim _{\rho \rightarrow 0} \frac{\mathcal{I}_{\infty}\left(u ; Q^{\prime}\left(x_{0}, \rho\right)\right)}{\rho^{2}}, \quad \text { for } \mathcal{L}^{2} \text {-a.e. } x_{0} \in \omega
$$

and $g$ is the Radon-Nikodym derivative of $\mathcal{I}_{\infty}(u ; \cdot)$ with respect to $\mathcal{H}_{[S(u)}^{1}$,

$$
g\left(x_{0}\right)=\lim _{\rho \rightarrow 0} \frac{\mathcal{I}_{\infty}\left(u ; Q^{\prime}\left(x_{0}, \rho\right)\right)}{\mathcal{H}^{1}\left(S(u) \cap Q^{\prime}\left(x_{0}, \rho\right)\right)}, \quad \text { for } \mathcal{H}^{1} \text {-a.e. } x_{0} \in S(u) .
$$

Now we would like to identify both densities $g$ and $h$. Note that we cannot use classical Integral Representation Theorems in $S B V$ (see e.g. Theorem 2.4 in [7] or Theorem 1 in [6]) because the term of surface energy does not grow linearly in the deformation jump. 


\subsection{Characterization of the $\Gamma$-limit}

\subsubsection{The upper bound}

We will proceed in two steps to prove the upper bound. Firstly, we will show that the inequality holds for deformations belonging to $L^{\infty}\left(\Omega ; \mathbb{R}^{3}\right)$ (see Lemma 3.7 below). Indeed, for those, we will use the integral representation proved above. In fact, we will show, with the help of a blow up argument, that the inequality holds separately for the surface and the bulk terms. Then, we will prove the inequality in its full generality in Lemma 3.8, using a truncation argument as in the proof of Lemma 3.3.

Lemma 3.7. For all $u \in B V\left(\Omega ; \mathbb{R}^{3}\right) \cap L^{\infty}\left(\Omega ; \mathbb{R}^{3}\right), \mathcal{I}_{\infty}(u ; \omega) \leq \mathcal{J}(u ; \omega)$.

Proof. It is enough to consider the case where $\mathcal{J}(u ; \omega)<+\infty$ and thus $u \in S B V^{p}\left(\omega ; \mathbb{R}^{3}\right)$. Let $u \in L^{\infty}\left(\omega ; \mathbb{R}^{3}\right) \cap S B V^{p}\left(\omega ; \mathbb{R}^{3}\right)$, according to (3.17) and (3.2), we must show that $h\left(x_{0}\right) \leq 2 \mathcal{Q} W_{0}\left(\nabla_{\alpha} u\left(x_{0}\right)\right)$ for $\mathcal{L}^{2}$-a.e. $x_{0} \in \omega$ and $g\left(x_{0}\right) \leq 2$ for $\mathcal{H}^{1}$-a.e. $x_{0} \in S(u)$.

Let us first treat the surface term. We have for $\mathcal{H}^{1}$-a.e. $x_{0} \in S(u)$,

$$
\begin{aligned}
g\left(x_{0}\right) & =\lim _{\rho \rightarrow 0} \frac{\mathcal{I}_{\infty}\left(u ; Q^{\prime}\left(x_{0}, \rho\right)\right)}{\mathcal{H}^{1}\left(S(u) \cap Q^{\prime}\left(x_{0}, \rho\right)\right)} \\
& \leq \limsup _{\rho \rightarrow 0} \frac{1}{\mathcal{H}^{1}\left(S(u) \cap Q^{\prime}\left(x_{0}, \rho\right)\right)}\left[2 \int_{Q^{\prime}\left(x_{0}, \rho\right)} W\left(\nabla_{\alpha} u \mid 0\right) d x_{\alpha}+2 \mathcal{H}^{1}\left(S(u) \cap Q^{\prime}\left(x_{0}, \rho\right)\right)\right] \\
& =\limsup _{\rho \rightarrow 0} \frac{\mu\left(Q^{\prime}\left(x_{0}, \rho\right)\right)}{\mathcal{H}^{1}\left(S(u) \cap Q^{\prime}\left(x_{0}, \rho\right)\right)}+2,
\end{aligned}
$$

where we set $\mu:=2 W\left(\nabla_{\alpha} u \mid 0\right) \mathcal{L}^{2}$. But since $\mu$ and $\mathcal{H}_{\lfloor S(u)}^{1}$ are mutually singular, we have for $\mathcal{H}^{1}$-a.e. $x_{0} \in S(u)$

$$
\lim _{\rho \rightarrow 0} \frac{\mu\left(Q^{\prime}\left(x_{0}, \rho\right)\right)}{\mathcal{H}^{1}\left(S(u) \cap Q^{\prime}\left(x_{0}, \rho\right)\right)}=0 .
$$

This shows that $g\left(x_{0}\right) \leq 2$ for $\mathcal{H}^{1}$-a.e. $x_{0} \in S(u)$.

Concerning the bulk term, choose $x_{0} \in \omega$ such that

$$
\lim _{\rho \rightarrow 0} f_{Q^{\prime}\left(x_{0}, \rho\right)}\left|\nabla_{\alpha} u\left(x_{\alpha}\right)-\nabla_{\alpha} u\left(x_{0}\right)\right|^{p} d x=0 .
$$

and

$$
\lim _{\rho \rightarrow 0} \frac{\mathcal{H}^{1}\left(S(u) \cap Q^{\prime}\left(x_{0}, \rho\right)\right)}{\rho^{2}}=0 .
$$

Since $\nabla_{\alpha} u \in L^{p}\left(\omega ; \mathbb{R}^{3 \times 2}\right)$ and the measures $\mathcal{L}^{2}$ and $\mathcal{H}_{[S(u)}^{1}$ are mutually singular, it follows that $\mathcal{L}^{2}$-a.e. $x_{0} \in \omega$ satisfies (3.18) and (3.19). For every $\rho>0$, Theorem 2 in [22] implies the existence of a sequence $\left\{v_{n}^{\rho}\right\} \subset W^{1, p}\left(Q^{\prime}\left(x_{0}, \rho\right) \times I ; \mathbb{R}^{3}\right)$ such that $v_{n}^{\rho} \rightarrow \nabla_{\alpha} u\left(x_{0}\right) \cdot x_{\alpha}$ in $L^{p}\left(Q^{\prime}\left(x_{0}, \rho\right) \times I ; \mathbb{R}^{3}\right)$ (thus a fortiori in $\left.L^{1}\left(Q^{\prime}\left(x_{0}, \rho\right) \times I ; \mathbb{R}^{3}\right)\right)$ and

$$
\int_{Q^{\prime}\left(x_{0}, \rho\right) \times I} W\left(\nabla_{\alpha} v_{n}^{\rho} \mid \frac{1}{\varepsilon_{n}} \nabla_{3} v_{n}^{\rho}\right) d x \rightarrow 2 \rho^{2} \mathcal{Q} W_{0}\left(\nabla_{\alpha} u\left(x_{0}\right)\right) .
$$

Moreover, by the construction of this recovery sequence (see [22]), there is no loss of generality in assuming that $\left\{v_{n}^{\rho}\right\}$ further satisfies

$$
\sup _{\rho>0, n \in \mathbb{N}}\left\|v_{n}^{\rho}\right\|_{L^{\infty}\left(Q^{\prime}\left(x_{0}, \rho\right) \times I ; \mathbb{R}^{3}\right)}<+\infty .
$$


QUASISTATIC EVOLUtion OF A BRITTLE THIN FILM

From the coercivity condition (2.1), we get

$$
M:=\sup _{\rho>0, n \in \mathbb{N}} f_{Q^{\prime}\left(x_{0}, \rho\right) \times I}\left|\left(\nabla_{\alpha} v_{n}^{\rho} \mid \frac{1}{\varepsilon_{n}} \nabla_{3} v_{n}^{\rho}\right)\right|^{p} d x<+\infty .
$$

Define $u_{n}^{\rho}(x):=u\left(x_{\alpha}\right)+v_{n}^{\rho}\left(x_{\alpha}, x_{3}\right)-\nabla_{\alpha} u\left(x_{0}\right) \cdot x_{\alpha}$. Then,

$$
u_{n}^{\rho} \rightarrow u \text { in } L^{1}\left(Q^{\prime}\left(x_{0}, \rho\right) \times I ; \mathbb{R}^{3}\right) \text { as } n \rightarrow+\infty, \sup _{\rho>0, n \in \mathbb{N}}\left\|u_{n}^{\rho}\right\|_{L^{\infty}\left(Q^{\prime}\left(x_{0}, \rho\right) \times I ; \mathbb{R}^{3}\right)}<+\infty
$$

and $S\left(u_{n}^{\rho}\right) \cap\left[Q^{\prime}\left(x_{0}, \rho\right) \times I\right]=\left[S(u) \cap Q^{\prime}\left(x_{0}, \rho\right)\right] \times I$. Thus,

$$
\begin{gathered}
\frac{\mathcal{I}_{\infty}\left(u ; Q^{\prime}\left(x_{0}, \rho\right)\right)}{\rho^{2}} \leq \liminf _{n \rightarrow+\infty} \frac{1}{\rho^{2}}\left\{\int_{Q^{\prime}\left(x_{0}, \rho\right) \times I} W\left(\nabla_{\alpha} u_{n}^{\rho} \mid \frac{1}{\varepsilon_{n}} \nabla_{3} u_{n}^{\rho}\right) d x\right. \\
\left.\quad+\int_{S\left(u_{n}^{\rho}\right) \cap\left[Q^{\prime}\left(x_{0}, \rho\right) \times I\right]}\left|\left(\left(\nu_{u_{n}^{\rho}}\right)_{\alpha} \mid \frac{1}{\varepsilon_{n}}\left(\nu_{u_{n}^{\rho}}\right)_{3}\right)\right| d \mathcal{H}^{2}\right\} \\
\leq \liminf _{n \rightarrow+\infty} \frac{1}{\rho^{2}} \int_{Q^{\prime}\left(x_{0}, \rho\right) \times I} W\left(\nabla_{\alpha} u\left(x_{\alpha}\right)-\nabla_{\alpha} u\left(x_{0}\right)+\nabla_{\alpha} v_{n}^{\rho}(x) \mid \frac{1}{\varepsilon_{n}} \nabla_{3} v_{n}^{\rho}(x)\right) d x \\
+2 \frac{\mathcal{H}^{1}\left(S(u) \cap Q^{\prime}\left(x_{0}, \rho\right)\right)}{\rho^{2}} .
\end{gathered}
$$

Thus from (3.19), we obtain

$$
h\left(x_{0}\right) \leq \liminf _{\rho \rightarrow 0} \liminf _{n \rightarrow+\infty} \frac{1}{\rho^{2}} \int_{Q^{\prime}\left(x_{0}, \rho\right) \times I} W\left(\nabla_{\alpha} u\left(x_{\alpha}\right)-\nabla_{\alpha} u\left(x_{0}\right)+\nabla_{\alpha} v_{n}^{\rho}(x) \mid \frac{1}{\varepsilon_{n}} \nabla_{3} v_{n}^{\rho}(x)\right) d x .
$$

Relations (3.11), (3.20), (3.21) and Hölder's inequality yield

$$
\begin{aligned}
h\left(x_{0}\right) \leq \liminf _{\rho \rightarrow 0} \liminf _{n \rightarrow+\infty} \frac{1}{\rho^{2}}\left\{\int_{Q^{\prime}\left(x_{0}, \rho\right) \times I} W\left(\nabla_{\alpha} v_{n}^{\rho} \mid \frac{1}{\varepsilon_{n}} \nabla_{3} v_{n}^{\rho}\right) d x\right. \\
+C \int_{Q^{\prime}\left(x_{0}, \rho\right) \times I}\left(1+\left|\nabla_{\alpha} u\left(x_{\alpha}\right)-\nabla_{\alpha} u\left(x_{0}\right)\right|^{p-1}\right. \\
\left.\left.\quad+\left|\left(\nabla_{\alpha} v_{n}^{\rho}(x) \mid \frac{1}{\varepsilon_{n}} \nabla_{3} v_{n}^{\rho}(x)\right)\right|^{p-1}\right)\left|\nabla_{\alpha} u\left(x_{\alpha}\right)-\nabla_{\alpha} u\left(x_{0}\right)\right| d x\right\} \\
\leq 2 \mathcal{Q} W_{0}\left(\nabla_{\alpha} u\left(x_{0}\right)\right)+C \limsup _{\rho \rightarrow 0}\left(f_{Q^{\prime}\left(x_{0}, \rho\right)}\left|\nabla_{\alpha} u\left(x_{\alpha}\right)-\nabla_{\alpha} u\left(x_{0}\right)\right|^{p} d x_{\alpha}\right) \\
+C\left(1+M^{(p-1) / p}\right) \limsup _{\rho \rightarrow 0}\left(f_{Q^{\prime}\left(x_{0}, \rho\right)}\left|\nabla_{\alpha} u\left(x_{\alpha}\right)-\nabla_{\alpha} u\left(x_{0}\right)\right|^{p} d x_{\alpha}\right)^{1 / p} .
\end{aligned}
$$

Thanks to (3.18), we conclude that $h\left(x_{0}\right) \leq 2 \mathcal{Q} W_{0}\left(\nabla_{\alpha} u\left(x_{0}\right)\right)$ for $\mathcal{L}^{2}$-a.e. $x_{0} \in \omega$.

Let us now turn back to the general case.

Lemma 3.8. For all $u \in B V\left(\Omega ; \mathbb{R}^{3}\right), \mathcal{I}(u ; \omega) \leq \mathcal{J}(u ; \omega)$.

Proof. As in the proof of Lemma 3.7, we can assume without loss of generality that $\mathcal{J}(u ; \omega)<+\infty$ and thus that $u \in S B V^{p}\left(\omega ; \mathbb{R}^{3}\right)$. In particular, it implies that $\mathcal{I}(u ; \omega)<+\infty$. Let $\varphi_{i} \in \mathcal{C}_{c}^{1}\left(\mathbb{R}^{3} ; \mathbb{R}^{3}\right)$ be the 
truncation function introduced in Lemma 3.3 and defined by (3.5). The Chain Rule formula, Theorem 3.96 in [2], implies that $\varphi_{i}(u) \in S B V^{p}\left(\omega ; \mathbb{R}^{3}\right) \cap L^{\infty}\left(\omega ; \mathbb{R}^{3}\right)$ and

$$
\left\{\begin{array}{l}
\left\|\varphi_{i}(u)\right\|_{L^{\infty}\left(\omega ; \mathbb{R}^{3}\right)} \leq e^{i}, \\
S\left(\varphi_{i}(u)\right) \subset S(u), \\
\nabla_{\alpha}\left(\varphi_{i}\left(u\left(x_{\alpha}\right)\right)\right)=\nabla \varphi_{i}\left(u\left(x_{\alpha}\right)\right) \circ \nabla_{\alpha} u\left(x_{\alpha}\right) \quad \mathcal{L}^{2} \text {-a.e. in } \omega
\end{array}\right.
$$

and $\varphi_{i}(u) \rightarrow u$ in $L^{1}\left(\omega ; \mathbb{R}^{3}\right)$ as $i \rightarrow+\infty$. Since $\varphi_{i}(u) \in L^{\infty}\left(\omega ; \mathbb{R}^{3}\right) \cap S B V^{p}\left(\omega ; \mathbb{R}^{3}\right)$, it follows from Lemmas 3.3 and 3.7 that

$$
\mathcal{I}\left(\varphi_{i}(u) ; \omega\right)=\mathcal{I}_{\infty}\left(\varphi_{i}(u) ; \omega\right) \leq \mathcal{J}\left(\varphi_{i}(u) ; \omega\right)
$$

and by lower semicontinuity of $\mathcal{I}(\cdot ; \omega)$ with respect to the strong $L^{1}\left(\omega ; \mathbb{R}^{3}\right)$-convergence, we have

$$
\mathcal{I}(u ; \omega) \leq \liminf _{i \rightarrow+\infty} \mathcal{I}\left(\varphi_{i}(u) ; \omega\right) \leq \limsup _{i \rightarrow+\infty} \mathcal{J}\left(\varphi_{i}(u) ; \omega\right) .
$$

But, in view of $(3.22), \mathcal{H}^{1}\left(S\left(\varphi_{i}(u)\right)\right) \leq \mathcal{H}^{1}(S(u))$ and, thanks to (3.5),

$$
\int_{\omega} \mathcal{Q} W_{0}\left(\nabla_{\alpha}\left(\varphi_{i}(u)\right)\right) d x_{\alpha} \leq \int_{\left\{|u|<e^{i}\right\}} \mathcal{Q} W_{0}\left(\nabla_{\alpha} u\right) d x_{\alpha}+\beta \int_{\left\{|u| \geq e^{i}\right\}}\left(1+\left|\nabla_{\alpha} u\right|^{p}\right) d x_{\alpha}
$$

Thus,

$$
\begin{aligned}
\limsup _{i \rightarrow+\infty} & \mathcal{J}\left(\varphi_{i}(u) ; \omega\right) \\
\quad \leq & \limsup _{i \rightarrow+\infty}\left\{2 \int_{\omega} \mathcal{Q} W_{0}\left(\nabla_{\alpha} u\right) d x_{\alpha}+2 \beta \int_{\left\{|u| \geq e^{i}\right\}}\left(1+\left|\nabla_{\alpha} u\right|^{p}\right) d x_{\alpha}+2 \mathcal{H}^{1}(S(u))\right\} \\
& \leq 2 \int_{\omega} \mathcal{Q} W_{0}\left(\nabla_{\alpha} u\right) d x_{\alpha}+2 \mathcal{H}^{1}(S(u)) \\
& =\mathcal{J}(u ; \omega),
\end{aligned}
$$

where we have used the fact that, by Chebyshev's inequality, $\mathcal{L}^{2}\left(\left\{|u| \geq e^{i}\right\}\right) \leq\|u\|_{L^{1}\left(\omega ; \mathbb{R}^{3}\right)} / e^{i} \rightarrow 0$ as $i \rightarrow+\infty$. Gathering (3.23) and (3.24), we deduce that $\mathcal{I}(u ; \omega) \leq \mathcal{J}(u ; \omega)$ and this completes the proof of the Lemma.

\subsubsection{The lower bound}

Let us now prove the lower bound. The proof is essentially based on a lower semicontinuity result in $S B V^{p}\left(\Omega ; \mathbb{R}^{3}\right)$. The main difficulty remains to show that any deformation $u \in B V\left(\Omega ; \mathbb{R}^{3}\right)$ satisfying $\mathcal{I}(u ; \omega)<+\infty$ belongs in fact to $S B V^{p}\left(\omega ; \mathbb{R}^{3}\right)$.

Lemma 3.9. For all $u \in B V\left(\Omega ; \mathbb{R}^{3}\right), \mathcal{I}(u ; \omega) \geq \mathcal{J}(u ; \omega)$.

Proof. It is not restrictive to assume that $\mathcal{I}(u ; \omega)<+\infty$. By $\Gamma$-convergence, there exists a sequence $\left\{u_{n}\right\} \subset S B V^{p}\left(\Omega ; \mathbb{R}^{3}\right)$ such that $u_{n} \rightarrow u$ in $L^{1}\left(\Omega ; \mathbb{R}^{3}\right)$ and

$$
\lim _{n \rightarrow+\infty}\left[\int_{\Omega} W\left(\nabla_{\alpha} u_{n} \mid \frac{1}{\varepsilon_{n}} \nabla_{3} u_{n}\right) d x+\int_{S\left(u_{n}\right)}\left|\left(\left(\nu_{u_{n}}\right)_{\alpha} \mid \frac{1}{\varepsilon_{n}}\left(\nu_{u_{n}}\right)_{3}\right)\right| d \mathcal{H}^{2}\right]=\mathcal{I}(u ; \omega) .
$$

Let us show that $u \in S B V\left(\omega ; \mathbb{R}^{3}\right)$. We will use the truncation function $\varphi_{i} \in \mathcal{C}_{c}^{1}\left(\mathbb{R}^{3} ; \mathbb{R}^{3}\right)$ defined in (3.5). The Chain Rule formula, Theorem 3.96 in [2], implies that $\varphi_{i}\left(u_{n}\right) \in S B V^{p}\left(\Omega ; \mathbb{R}^{3}\right)$ and 


$$
\left\{\begin{array}{l}
\left\|\varphi_{i}\left(u_{n}\right)\right\|_{L^{\infty}\left(\Omega ; \mathbb{R}^{3}\right)} \leq e^{i}, \\
S\left(\varphi_{i}\left(u_{n}\right)\right) \subset S\left(u_{n}\right), \\
\nabla\left(\varphi_{i}\left(u_{n}\right)(x)\right)=\nabla \varphi_{i}\left(u_{n}(x)\right) \circ \nabla u_{n}(x) \quad \mathcal{L}^{3} \text {-a.e. in } \Omega .
\end{array}\right.
$$

As $\nu_{u_{n}}(x)= \pm \nu_{\varphi_{i}\left(u_{n}\right)}(x)$ for $\mathcal{H}^{2}$-a.e. $x \in S\left(\varphi_{i}\left(u_{n}\right)\right)$, we get

$$
\begin{array}{r}
\sup _{n \in \mathbb{N}}\left[\int_{\Omega}\left|\left(\nabla_{\alpha}\left(\varphi_{i}\left(u_{n}\right)\right) \mid \frac{1}{\varepsilon_{n}} \nabla_{3}\left(\varphi_{i}\left(u_{n}\right)\right)\right)\right|^{p} d x+\int_{S\left(\varphi_{i}\left(u_{n}\right)\right)}\left|\left(\left(\nu_{\varphi_{i}\left(u_{n}\right)}\right)_{\alpha} \mid \frac{1}{\varepsilon_{n}}\left(\nu_{\varphi_{i}\left(u_{n}\right)}\right)_{3}\right)\right| d \mathcal{H}^{2}\right] \\
\leq \sup _{n \in \mathbb{N}}\left[\int_{\Omega}\left|\left(\nabla_{\alpha} u_{n} \mid \frac{1}{\varepsilon_{n}} \nabla_{3} u_{n}\right)\right|^{p} d x+\int_{S\left(u_{n}\right)}\left|\left(\left(\nu_{u_{n}}\right)_{\alpha} \mid \frac{1}{\varepsilon_{n}}\left(\nu_{u_{n}}\right)_{3}\right)\right| d \mathcal{H}^{2}\right]<+\infty
\end{array}
$$

where we used (3.25) together with the coercivity condition (2.1). Lemma 2.2 and a diagonalization argument yield the existence of a subsequence (still denoted by $\left.\left\{\varepsilon_{n}\right\}\right)$, and a function $v_{i} \in S B V^{p}\left(\omega ; \mathbb{R}^{3}\right)$ such that $\varphi_{i}\left(u_{n}\right) \rightarrow v_{i}$ in $S B V^{p}\left(\Omega ; \mathbb{R}^{3}\right)$ as $n \rightarrow+\infty$. But, since $u_{n} \rightarrow u$ and $\varphi_{i}\left(u_{n}\right) \rightarrow \varphi_{i}(u)$ in $L^{1}\left(\Omega ; \mathbb{R}^{3}\right)$ as $n \rightarrow+\infty$, we deduce that $v_{i}=\varphi_{i}(u) \in S B V^{p}\left(\omega ; \mathbb{R}^{3}\right)$ for every $i \in \mathbb{N}$. By virtue of Theorem 3.96 in $[2]$,

$$
0=D^{c} v_{i}=\nabla \varphi_{i}(\tilde{u}) \circ D^{c} u \quad \text { in } \omega \backslash S(u),
$$

where $\tilde{u}$ denotes the approximate limit of $u$ at $x_{\alpha} \in \omega \backslash S(u)$. Define $E_{i}:=\left\{x_{\alpha} \in \omega \backslash S(u):\left|\tilde{u}\left(x_{\alpha}\right)\right|<e^{i}\right\}$, since $\tilde{u}$ is a Borel function and $S(u)$ is a Borel set (see Proposition 3.64 (a) in [2]), $E_{i}$ is a Borel set. Moreover, as $\left\{E_{i}\right\}$ is an increasing sequence of sets whose union is $\omega \backslash S(u)$, we get

$$
\left|D^{c} u\right|(\omega)=\left|D^{c} u\right|(\omega \backslash S(u))=\lim _{i \rightarrow+\infty}\left|D^{c} u\right|\left(E_{i}\right)=\lim _{i \rightarrow+\infty}\left|\nabla \varphi_{i}(\tilde{u}) \circ D^{c} u\right|\left(E_{i}\right)=\lim _{i \rightarrow+\infty}\left|D^{c} v_{i}\right|\left(E_{i}\right)=0,
$$

where we have used the fact that $\nabla \varphi_{i}\left(\tilde{u}\left(x_{\alpha}\right)\right)=\operatorname{Id}$ for all $x_{\alpha} \in E_{i}$. Thus $u \in S B V\left(\omega ; \mathbb{R}^{3}\right)$ and by (3.25), Remark 3.2, Theorem 5.29 in [2] and Theorem 3.7 in [1]

$$
\begin{aligned}
\mathcal{I}(u ; \omega) & \geq \liminf _{n \rightarrow+\infty}\left[\int_{\Omega} \mathcal{Q} W_{0}\left(\nabla_{\alpha} u_{n}\right) d x+\mathcal{H}^{2}\left(S\left(u_{n}\right)\right)\right] \\
& \geq 2 \int_{\omega} \mathcal{Q} W_{0}\left(\nabla_{\alpha} u\right) d x_{\alpha}+2 \mathcal{H}^{1}(S(u)) .
\end{aligned}
$$

In particular, the $p$-coercivity of $\mathcal{Q} W_{0}$ implies that $u \in S B V^{p}\left(\omega ; \mathbb{R}^{3}\right)$ and thus, according to (3.2), that $\mathcal{I}(u ; \omega) \geq \mathcal{J}(u, \omega)$.

Proof of Theorem 3.1. We have shown that for any sequence $\left\{\varepsilon_{j}\right\} \searrow 0^{+}$, there exists a further subsequence $\left\{\varepsilon_{j_{n}}\right\} \equiv\left\{\varepsilon_{n}\right\}$ such that $\mathcal{I}_{\varepsilon_{n}}(\cdot ; \omega) \Gamma$-converges to $\mathcal{I}(\cdot ; \omega)$ for the strong $L^{1}\left(\Omega ; \mathbb{R}^{3}\right)$-topology. By virtue of Lemmas 3.8 and 3.9 , we have $\mathcal{I}(\cdot ; \omega)=\mathcal{J}(\cdot ; \omega)$. Since the $\Gamma$-limit does not depend upon the extracted subsequence, we deduce, in the light of Proposition 8.3 in [12], that the whole sequence $\mathcal{I}_{\varepsilon}(\cdot ; \omega) \Gamma$-converges to $\mathcal{J}(\cdot ; \omega)$.

\subsection{Boundary conditions}

Let us now deal with boundary condition constraints that will be of use in Lemmas 5.2 and 5.5 in order to prove the minimality property of the limit quasistatic evolution. Indeed, it will allow to extend functions on the enlarged cylinder $\Omega^{\prime}$ by the value of the boundary condition. The following result, very close in spirit to Lemma 2.6 in [10], relies on De Giorgi's slicing argument together with the fact that we can 


\section{JEAN-FranÇOIS BABADJIAN}

consider cut-off functions depending only on $x_{\alpha}$ (see also the proof of Lemma 3.6). It is established that any recovery sequence can be chosen so as to match the lateral boundary condition of its target.

We recall that $\omega^{\prime}$ is a bounded open subset of $\mathbb{R}^{2}$ containing $\omega$ and that $\Omega^{\prime}=\omega^{\prime} \times I$. In all that follows, if $v \in S B V^{p}\left(\Omega^{\prime} ; \mathbb{R}^{3}\right)$, we will denote by $v^{-}$(resp. $v^{+}$) the inner (resp. outer) trace of $v$ on $\partial \omega \times I$.

Lemma 3.10. For every $u \in S B V^{p}\left(\omega ; \mathbb{R}^{3}\right) \cap L^{\infty}\left(\omega ; \mathbb{R}^{3}\right)$, there exists a sequence $\left\{\bar{u}_{\varepsilon}\right\} \subset S B V^{p}\left(\Omega ; \mathbb{R}^{3}\right)$ such that $\bar{u}_{\varepsilon} \rightarrow u$ in $L^{p}\left(\Omega ; \mathbb{R}^{3}\right), \bar{u}_{\varepsilon}=u$ in a neighborhood of $\partial \omega \times I$ and

$$
\mathcal{J}(u)=\lim _{\varepsilon \rightarrow 0}\left[\int_{\Omega} W\left(\nabla_{\alpha} \bar{u}_{\varepsilon} \mid \frac{1}{\varepsilon} \nabla_{3} \bar{u}_{\varepsilon}\right) d x+\int_{S\left(\bar{u}_{\varepsilon}\right)}\left|\left(\left(\nu_{\bar{u}_{\varepsilon}}\right)_{\alpha} \mid \frac{1}{\varepsilon}\left(\nu_{\bar{u}_{\varepsilon}}\right)_{3}\right)\right| d \mathcal{H}^{2}\right] .
$$

Proof. According to Theorem 3.1 and Lemma 3.3, there exists a sequence $\left\{u_{\varepsilon}\right\} \subset S B V^{p}\left(\Omega ; \mathbb{R}^{3}\right)$ strongly converging to $u$ in $L^{1}\left(\Omega ; \mathbb{R}^{3}\right)$, satisfying $\sup _{\varepsilon>0}\left\|u_{\varepsilon}\right\|_{L^{\infty}\left(\Omega ; \mathbb{R}^{3}\right)}<+\infty$ and

$$
\mathcal{J}(u)=\lim _{\varepsilon \rightarrow 0}\left[\int_{\Omega} W\left(\nabla_{\alpha} u_{\varepsilon} \mid \frac{1}{\varepsilon} \nabla_{3} u_{\varepsilon}\right) d x+\int_{S\left(u_{\varepsilon}\right)}\left|\left(\left(\nu_{u_{\varepsilon}}\right)_{\alpha} \mid \frac{1}{\varepsilon}\left(\nu_{u_{\varepsilon}}\right)_{3}\right)\right| d \mathcal{H}^{2}\right] .
$$

In particular, $u_{\varepsilon} \rightarrow u$ in $L^{p}\left(\Omega ; \mathbb{R}^{3}\right)$ and from the $p$-coercivity condition $(2.1)$, it follows that

$$
C:=\sup _{\varepsilon>0}\left[\int_{\Omega}\left(1+\left|\left(\nabla_{\alpha} u_{\varepsilon} \mid \frac{1}{\varepsilon} \nabla_{3} u_{\varepsilon}\right)\right|^{p}\right) d x+\int_{S\left(u_{\varepsilon}\right)}\left|\left(\left(\nu_{u_{\varepsilon}}\right)_{\alpha} \mid \frac{1}{\varepsilon}\left(\nu_{u_{\varepsilon}}\right)_{3}\right)\right| d \mathcal{H}^{2}\right]<+\infty .
$$

Set

$$
K_{\varepsilon}:=\llbracket \frac{1}{\left\|u_{\varepsilon}-u\right\|_{L^{p}\left(\Omega ; \mathbb{R}^{3}\right)}^{1 / 2}} \rrbracket, \quad M_{\varepsilon}:=\llbracket \sqrt{K_{\varepsilon}} \rrbracket
$$

and denote

$$
\omega(\varepsilon):=\left\{x_{\alpha} \in \omega: \operatorname{dist}\left(x_{\alpha}, \partial \omega\right)<\frac{M_{\varepsilon}}{K_{\varepsilon}}\right\} \quad \text { and } \quad \omega_{i}^{\varepsilon}:=\left\{x_{\alpha} \in \omega: \operatorname{dist}\left(x_{\alpha}, \partial \omega\right) \in\left[\frac{i}{K_{\varepsilon}}, \frac{i+1}{K_{\varepsilon}}\right)\right\},
$$

for all $i \in\left\{0, \ldots, M_{\varepsilon}-1\right\}$. From (3.26), we get the existence of a $i(\varepsilon) \in\left\{0, \ldots, M_{\varepsilon}-1\right\}$ such that

$$
\int_{\omega_{i(\varepsilon)}^{\varepsilon} \times I}\left(1+\left|\left(\nabla_{\alpha} u_{\varepsilon} \mid \frac{1}{\varepsilon} \nabla_{3} u_{\varepsilon}\right)\right|^{p}\right) d x+\int_{S\left(u_{\varepsilon}\right) \cap\left[\omega_{i(\varepsilon)}^{\varepsilon} \times I\right]}\left|\left(\left(\nu_{u_{\varepsilon}}\right)_{\alpha} \mid \frac{1}{\varepsilon}\left(\nu_{u_{\varepsilon}}\right)_{3}\right)\right| d \mathcal{H}^{2} \leq \frac{C}{M_{\varepsilon}} .
$$

Let us now consider a cut-off function $\phi_{\varepsilon} \in \mathcal{C}_{c}^{\infty}(\omega ;[0,1])$ independent of $x_{3}$ and satisfying

$$
\phi_{\varepsilon}\left(x_{\alpha}\right)=\left\{\begin{array}{ll}
1 \quad \text { if } \quad \operatorname{dist}\left(x_{\alpha}, \partial \omega\right)>\frac{i(\varepsilon)+1}{K_{\varepsilon}}, \\
0 \quad \text { if } \quad \operatorname{dist}\left(x_{\alpha}, \partial \omega\right) \leq \frac{i(\varepsilon)}{K_{\varepsilon}}
\end{array} \quad \text { and } \quad\left\|\nabla_{\alpha} \phi_{\varepsilon}\right\|_{L^{\infty}(\omega)} \leq 2 K_{\varepsilon} .\right.
$$

Define $\bar{u}_{\varepsilon}(x):=\phi_{\varepsilon}\left(x_{\alpha}\right) u_{\varepsilon}(x)+\left(1-\phi_{\varepsilon}\left(x_{\alpha}\right)\right) u\left(x_{\alpha}\right)$; then $\bar{u}_{\varepsilon} \in S B V^{p}\left(\Omega ; \mathbb{R}^{3}\right), \bar{u}_{\varepsilon} \rightarrow u$ in $L^{p}\left(\Omega ; \mathbb{R}^{3}\right), \bar{u}_{\varepsilon}=u$ 
QuASistatic EVOLUTION OF A BRITTLE THIN FILM

in a neighborhood of $\partial \omega \times I$ and $S\left(\bar{u}_{\varepsilon}\right) \subset S\left(u_{\varepsilon}\right) \cup(S(u) \times I)$. The $p$-growth condition (2.1) implies that

$$
\begin{aligned}
\mathcal{J}(u) \geq & \limsup _{\varepsilon \rightarrow 0}\left[\int_{\left\{x_{\alpha} \in \omega: \operatorname{dist}\left(x_{\alpha}, \partial \omega\right)>\frac{i(\varepsilon)+1}{K_{\varepsilon}}\right\} \times I} W\left(\nabla_{\alpha} \bar{u}_{\varepsilon} \mid \frac{1}{\varepsilon} \nabla_{3} \bar{u}_{\varepsilon}\right) d x\right. \\
& \left.\quad+\int_{S\left(\bar{u}_{\varepsilon}\right) \cap\left[\left\{x_{\alpha} \in \omega: \operatorname{dist}\left(x_{\alpha}, \partial \omega\right)>\frac{i(\varepsilon)+1}{K_{\varepsilon}}\right\} \times I\right]}\left|\left(\left(\nu_{\bar{u}_{\varepsilon}}\right)_{\alpha} \mid \frac{1}{\varepsilon}\left(\nu_{\bar{u}_{\varepsilon}}\right)_{3}\right)\right| d \mathcal{H}^{2}\right] \\
\geq & \limsup _{\varepsilon \rightarrow 0}\left[\int_{\Omega} W\left(\nabla_{\alpha} \bar{u}_{\varepsilon} \mid \frac{1}{\varepsilon} \nabla_{3} \bar{u}_{\varepsilon}\right) d x+\int_{S\left(\bar{u}_{\varepsilon}\right)}\left|\left(\left(\nu_{\bar{u}_{\varepsilon}}\right)_{\alpha} \mid \frac{1}{\varepsilon}\left(\nu_{\bar{u}_{\varepsilon}}\right)_{3}\right)\right| d \mathcal{H}^{2}\right. \\
& -2 \beta \int_{\left\{x_{\alpha} \in \omega: \operatorname{dist}\left(x_{\alpha}, \partial \omega\right) \leq \frac{i(\varepsilon)}{K_{\varepsilon}}\right\}}\left(1+\left|\nabla_{\alpha} u\right|^{p}\right) d x_{\alpha} \\
& -c_{1} K_{\varepsilon}^{p} \int_{\omega_{i(\varepsilon)}^{\varepsilon} \times I}\left|u_{\varepsilon}-u\right|^{p} d x-\mathcal{H}_{2} \int_{\omega_{i(\varepsilon)}^{\varepsilon} \times I}\left(S(u) \cap\left\{x_{\alpha} \in \omega: \operatorname{dist}\left(x_{\alpha}, \partial \omega\right) \leq \frac{i(\varepsilon)}{K_{\varepsilon}}\right\}\right) \\
\geq & \left.\left.\quad-c_{3} \int_{S\left(u_{\varepsilon}\right) \cap\left[\omega_{i(\varepsilon)}^{\varepsilon} \times I\right]}\left|\left(\left(\nu_{u_{\varepsilon}}\right)_{\alpha} \mid \frac{1}{\varepsilon}\left(\nu_{u_{\varepsilon}}\right)_{3}\right)\right| d \mathcal{H}^{2}-c_{4} \mathcal{H}^{1}\left(S(u) \cap \omega_{i(\varepsilon)}^{\varepsilon}\right)\right]\left.\left(\nabla_{\alpha} u_{\varepsilon} \mid \frac{1}{\varepsilon} \nabla_{3} u_{\varepsilon}\right)\right|^{p}\right) d x \\
& \quad \limsup _{\varepsilon \rightarrow 0}\left[\int_{\Omega} W\left(\nabla_{\alpha} \bar{u}_{\varepsilon} \mid \frac{1}{\varepsilon} \nabla_{3} \bar{u}_{\varepsilon}\right) d x+\int_{S\left(\bar{u}_{\varepsilon}\right)}\left|\left(\left(\nu_{\bar{u}_{\varepsilon}}\right)_{\alpha} \mid \frac{1}{\varepsilon}\left(\nu_{\bar{u}_{\varepsilon}}\right)_{3}\right)\right| d \mathcal{H}^{2}\right. \\
& \left.-c\left(\int_{\omega(\varepsilon)}\left(1+\left|\nabla_{\alpha} u\right|^{p}\right) d x_{\alpha}+\mathcal{H}^{1}(S(u) \cap \omega(\varepsilon))+\left\|u_{\varepsilon}-u\right\|_{L^{p}\left(\Omega ; \mathbb{R}^{3}\right)}^{p / 2}+\frac{1}{M_{\varepsilon}}\right)\right],
\end{aligned}
$$

where we have used (3.27) in the last inequality. Thus, since $M_{\varepsilon} \rightarrow+\infty, M_{\varepsilon} / K_{\varepsilon} \rightarrow 0$ and $\mathcal{H}^{1}(S(u))<$ $+\infty$, we obtain from the previous relation and the $\Gamma$-lim inf inequality

$$
\mathcal{J}(u)=\lim _{\varepsilon \rightarrow 0}\left[\int_{\Omega} W\left(\nabla_{\alpha} \bar{u}_{\varepsilon} \mid \frac{1}{\varepsilon} \nabla_{3} \bar{u}_{\varepsilon}\right) d x+\int_{S\left(\bar{u}_{\varepsilon}\right)}\left|\left(\left(\nu_{\bar{u}_{\varepsilon}}\right)_{\alpha} \mid \frac{1}{\varepsilon}\left(\nu_{\bar{u}_{\varepsilon}}\right)_{3}\right)\right| d \mathcal{H}^{2}\right] .
$$

Let us now state a $\Gamma$-convergence result involving the boundary conditions. Consider a sequence of boundary conditions $\left\{g_{\varepsilon}\right\} \subset W^{1, p}\left(\Omega^{\prime} ; \mathbb{R}^{3}\right)$, and let $g \in W^{1, p}\left(\omega^{\prime} ; \mathbb{R}^{3}\right)$ and $H \in L^{p}\left(\Omega^{\prime} ; \mathbb{R}^{3}\right)$ be such that

$$
\left\{\begin{array}{l}
\sup _{\varepsilon>0}\left\|g_{\varepsilon}\right\|_{L^{\infty}\left(\Omega^{\prime} ; \mathbb{R}^{3}\right)}<+\infty, \\
g_{\varepsilon} \rightarrow g \text { in } W^{1, p}\left(\Omega^{\prime} ; \mathbb{R}^{3}\right), \quad \frac{1}{\varepsilon} \nabla_{3} g_{\varepsilon} \rightarrow H \text { in } L^{p}\left(\Omega^{\prime} ; \mathbb{R}^{3}\right) .
\end{array}\right.
$$

Then,

Corollary 3.11. The functional $\mathcal{I}_{\varepsilon}^{g_{\varepsilon}}: B V\left(\Omega^{\prime} ; \mathbb{R}^{3}\right) \rightarrow \overline{\mathbb{R}}$ defined by

$$
\mathcal{I}_{\varepsilon}^{g_{\varepsilon}}(u):=\left\{\begin{array}{cc}
\int_{\Omega} W\left(\nabla_{\alpha} u \mid \frac{1}{\varepsilon} \nabla_{3} u\right) d x+\int_{S(u)}\left|\left(\left(\nu_{u}\right)_{\alpha} \mid \frac{1}{\varepsilon}\left(\nu_{u}\right)_{3}\right)\right| d \mathcal{H}^{2} & \text { if }\left\{\begin{array}{l}
u \in S B V^{p}\left(\Omega^{\prime} ; \mathbb{R}^{3}\right), \\
u=g_{\varepsilon} \text { on }\left[\omega^{\prime} \backslash \bar{\omega}\right] \times I,
\end{array}\right. \\
+\infty & \text { otherwise }
\end{array}\right.
$$




\section{JEAN-FranÇOIS BABADJIAN}

$\Gamma$-converges for the strong $L^{1}\left(\Omega^{\prime} ; \mathbb{R}^{3}\right)$-topology towards $\mathcal{J}^{g}: B V\left(\Omega^{\prime} ; \mathbb{R}^{3}\right) \rightarrow \overline{\mathbb{R}}$ defined by

$$
\mathcal{J}^{g}(u):=\left\{\begin{array}{cl}
2 \int_{\omega} \mathcal{Q} W_{0}\left(\nabla_{\alpha} u\right) d x_{\alpha}+2 \mathcal{H}^{1}(S(u)) & \text { if }\left\{\begin{array}{l}
u \in S B V^{p}\left(\omega^{\prime} ; \mathbb{R}^{3}\right), \\
u=g \text { on } \omega^{\prime} \backslash \bar{\omega},
\end{array}\right. \\
+\infty & \text { otherwise. }
\end{array}\right.
$$

Remark 3.12. Note that in the statement of the previous Corollary, the bulk integrals are still computed over $\Omega$ (resp. $\omega$ ) as in Theorem 3.1, however, since the jump set of the deformations can now reach the lateral boundary $\partial \omega \times I$, the surface integrals are implicitly computed over $\bar{\omega} \times I$ (resp. $\bar{\omega}$ ) or equivalently $\Omega^{\prime}\left(\right.$ resp. $\left.\omega^{\prime}\right)$.

Proof. Let us first prove the $\Gamma$ - liminf inequality. Consider a sequence $\left\{u_{\varepsilon}\right\} \subset L^{1}\left(\Omega^{\prime} ; \mathbb{R}^{3}\right)$ strongly converging to $u$ in $L^{1}\left(\Omega^{\prime} ; \mathbb{R}^{3}\right)$. It is not restrictive to assume that

$$
\liminf _{\varepsilon \rightarrow 0} \mathcal{I}_{\varepsilon}^{g_{\varepsilon}}\left(u_{\varepsilon}\right)<+\infty
$$

Then, for a (not relabeled) subsequence, $u_{\varepsilon} \in S B V^{p}\left(\Omega^{\prime} ; \mathbb{R}^{3}\right), u_{\varepsilon}=g_{\varepsilon}$ on $\left[\omega^{\prime} \backslash \bar{\omega}\right] \times I$ and arguing as in the proof of Lemma 3.9, we get that $u \in S B V^{p}\left(\omega^{\prime} ; \mathbb{R}^{3}\right)$. Consequently, since $u=g$ on $\omega^{\prime} \backslash \bar{\omega}$, we get from Theorem 3.1 and the definition of $\mathcal{J}^{g}$ that

$$
\mathcal{J}^{g}(u) \leq \liminf _{\varepsilon \rightarrow 0} \mathcal{I}_{\varepsilon}^{g_{\varepsilon}}\left(u_{\varepsilon}\right) .
$$

Let $u \in S B V^{p}\left(\omega^{\prime} ; \mathbb{R}^{3}\right)$ satisfying $u=g$ on $\omega^{\prime} \backslash \bar{\omega}$. It remains to construct a recovery sequence. We first assume that $u \in L^{\infty}\left(\omega^{\prime} ; \mathbb{R}^{3}\right)$. Then, by virtue of Lemma 3.10 , there exists a sequence $\left\{\bar{u}_{\varepsilon}\right\} \subset S B V^{p}\left(\Omega ; \mathbb{R}^{3}\right)$ satisfying $\bar{u}_{\varepsilon} \rightarrow u$ in $L^{1}\left(\Omega ; \mathbb{R}^{3}\right), \bar{u}_{\varepsilon}=u$ in a neighborhood of $\partial \omega \times I$ and

$$
\begin{aligned}
& 2 \int_{\omega} \mathcal{Q} W_{0}\left(\nabla_{\alpha} u\right) d x_{\alpha}+2 \mathcal{H}^{1}(S(u) \cap \omega) \\
& \quad=\lim _{\varepsilon \rightarrow 0}\left[\int_{\Omega} W\left(\nabla_{\alpha} \bar{u}_{\varepsilon} \mid \frac{1}{\varepsilon} \nabla_{3} \bar{u}_{\varepsilon}\right) d x+\int_{S\left(\bar{u}_{\varepsilon}\right) \cap \Omega}\left|\left(\left(\nu_{\bar{u}_{\varepsilon}}\right)_{\alpha} \mid \frac{1}{\varepsilon}\left(\nu_{\bar{u}_{\varepsilon}}\right)_{3}\right)\right| d \mathcal{H}^{2}\right] .
\end{aligned}
$$

Since $g \in W^{1, p}\left(\omega^{\prime} \backslash \bar{\omega} ; \mathbb{R}^{3}\right)$, by Corollary 3.89 in [2], the function

$$
v_{\varepsilon}:=\bar{u}_{\varepsilon} \chi_{\Omega}+g \chi_{\left[\omega^{\prime} \backslash \bar{\omega}\right] \times I} \in B V\left(\Omega^{\prime} ; \mathbb{R}^{3}\right)
$$

and, viewing $D \bar{u}_{\varepsilon}$ (resp. $D g$ ) as measures on all $\mathbb{R}^{3}$ and concentrated on $\Omega$ (resp. $\left[\omega^{\prime} \backslash \bar{\omega}\right] \times I$ ), we get as $v_{\varepsilon}^{-}=\bar{u}_{\varepsilon}^{-}=u^{-}$and $v_{\varepsilon}^{+}=g^{+}$on $\partial \omega \times I$

$$
D v_{\varepsilon}=D \bar{u}_{\varepsilon}+\left(g^{+}-\bar{u}^{-}\right) \otimes \nu_{\partial \omega \times I}+D g .
$$

In particular, we observe that $v_{\varepsilon} \in S B V^{p}\left(\Omega^{\prime} ; \mathbb{R}^{3}\right)$ and $v_{\varepsilon} \rightarrow u$ in $L^{1}\left(\Omega^{\prime} ; \mathbb{R}^{3}\right)$ but we may have created some additional jump set on $\partial \omega \times I$. However, $S\left(v_{\varepsilon}\right) \cap[\partial \omega \times I] \cong[S(u) \cap \partial \omega] \times I$, and since $\nu_{\partial \omega \times I}=\nu_{v_{\varepsilon}}$ $\mathcal{H}^{2}$-a.e. in $S\left(v_{\varepsilon}\right) \cap[\partial \omega \times I]$ and $\left(\nu_{\partial \omega \times I}\right)_{3}=0$,

$$
\int_{S\left(v_{\varepsilon}\right) \cap[\partial \omega \times I]}\left|\left(\left(\nu_{v_{\varepsilon}}\right)_{\alpha} \mid \frac{1}{\varepsilon}\left(\nu_{v_{\varepsilon}}\right)_{3}\right)\right| d \mathcal{H}^{2}=\mathcal{H}^{2}\left(S\left(v_{\varepsilon}\right) \cap[\partial \omega \times I]\right)=2 \mathcal{H}^{1}(S(u) \cap \partial \omega) .
$$

Replacing in (3.29), it yields

$$
\begin{aligned}
\mathcal{J}^{g}(u) & =2 \int_{\omega} \mathcal{Q} W_{0}\left(\nabla_{\alpha} u\right) d x_{\alpha}+2 \mathcal{H}^{1}(S(u)) \\
& =\lim _{\varepsilon \rightarrow 0}\left[\int_{\Omega} W\left(\nabla_{\alpha} v_{\varepsilon} \mid \frac{1}{\varepsilon} \nabla_{3} v_{\varepsilon}\right) d x+\int_{S\left(v_{\varepsilon}\right)}\left|\left(\left(\nu_{v_{\varepsilon}}\right)_{\alpha} \mid \frac{1}{\varepsilon}\left(\nu_{v_{\varepsilon}}\right)_{3}\right)\right| d \mathcal{H}^{2}\right] .
\end{aligned}
$$


QUASISTATIC EVOLUTION OF A BRITTLE THIN FILM

Actually, $v_{\varepsilon}=g$ on $\left[\omega^{\prime} \backslash \bar{\omega}\right] \times I$ so that we need to modify $v_{\varepsilon}$ in order it to have the value $g_{\varepsilon}$ instead of $g$ on the enlarged part of the domain. Let $H_{j} \in \mathcal{C}_{c}^{\infty}\left(\Omega ; \mathbb{R}^{3}\right)$ be a sequence strongly converging to $H$ in $L^{p}\left(\Omega ; \mathbb{R}^{3}\right)$ and extended by the value zero on $\left[\omega^{\prime} \backslash \bar{\omega}\right] \times I$, and set $b_{j}\left(x_{\alpha}, x_{3}\right):=\int_{-1}^{x_{3}} H_{j}\left(x_{\alpha}, s\right) d s$. We now define

$$
u_{\varepsilon}^{j}(x):=v_{\varepsilon}(x)-g\left(x_{\alpha}\right)+g_{\varepsilon}(x)-\varepsilon b_{j}(x) .
$$

It follows that $u_{\varepsilon}^{j} \in S B V^{p}\left(\Omega^{\prime} ; \mathbb{R}^{3}\right), u_{\varepsilon}^{j} \rightarrow u$ in $L^{1}\left(\Omega^{\prime} ; \mathbb{R}^{3}\right)$ as $\varepsilon \rightarrow 0, u_{\varepsilon}^{j}=g_{\varepsilon}$ on $\left[\omega^{\prime} \backslash \bar{\omega}\right] \times I$. Furthermore, since $u_{\varepsilon}^{j}$ is a smooth perturbation of $v_{\varepsilon}$ on the whole domain $\Omega^{\prime}$, both sequences have the same jump set, namely $S\left(u_{\varepsilon}^{j}\right) \cong S\left(u_{\varepsilon}\right)$, and consequently, the surface energy is not affected,

$$
\int_{S\left(u_{\varepsilon}^{j}\right)}\left|\left(\left(\nu_{u_{\varepsilon}^{j}}\right)_{\alpha} \mid \frac{1}{\varepsilon}\left(\nu_{u_{\varepsilon}^{j}}\right)_{3}\right)\right| d \mathcal{H}^{2}=\int_{S\left(v_{\varepsilon}\right)}\left|\left(\left(\nu_{v_{\varepsilon}}\right)_{\alpha} \mid \frac{1}{\varepsilon}\left(\nu_{v_{\varepsilon}}\right)_{3}\right)\right| d \mathcal{H}^{2} .
$$

Let us treat now the bulk energy. According to Remark 3.5, (3.11) and Hölder's Inequality, we have

$$
\begin{aligned}
& \int_{\Omega} W\left(\nabla_{\alpha} u_{\varepsilon}^{j} \mid \frac{1}{\varepsilon} \nabla_{3} u_{\varepsilon}^{j}\right) d x \\
& =\int_{\Omega} W\left(\nabla_{\alpha} v_{\varepsilon}-\nabla_{\alpha} g+\nabla_{\alpha} g_{\varepsilon}-\varepsilon \nabla_{\alpha} b_{j} \mid \frac{1}{\varepsilon} \nabla_{3} v_{\varepsilon}+\frac{1}{\varepsilon} \nabla_{3} g_{\varepsilon}-H_{j}\right) d x \\
& \leq \int_{\Omega} W\left(\nabla_{\alpha} v_{\varepsilon} \mid \frac{1}{\varepsilon} \nabla_{3} v_{\varepsilon}\right) d x+\beta \int_{\Omega}\left(1+\left|\left(\nabla_{\alpha} v_{\varepsilon} \mid \frac{1}{\varepsilon} \nabla_{3} v_{\varepsilon}\right)\right|^{p-1}+\left|\left(\nabla_{\alpha} g_{\varepsilon} \mid \frac{1}{\varepsilon} \nabla_{3} g_{\varepsilon}\right)\right|^{p-1}\right. \\
& \left.\quad+\left|\nabla_{\alpha} g\right|^{p-1}+\left|\left(\varepsilon \nabla_{\alpha} b_{j} \mid H_{j}\right)\right|^{p-1}\right)\left|\left(\nabla_{\alpha} g_{\varepsilon}-\nabla_{\alpha} g-\varepsilon \nabla_{\alpha} b_{j} \mid \frac{1}{\varepsilon} \nabla_{3} g_{\varepsilon}-H_{j}\right)\right| d x \\
& \leq \int_{\Omega} W\left(\nabla_{\alpha} v_{\varepsilon} \mid \frac{1}{\varepsilon} \nabla_{3} v_{\varepsilon}\right) d x+c\left(1+\left\|\left(\nabla_{\alpha} v_{\varepsilon} \mid \frac{1}{\varepsilon} \nabla_{3} v_{\varepsilon}\right)\right\|_{L^{p}\left(\Omega ; \mathbb{R}^{3 \times 3}\right)}^{p-1}+\left\|\left(\nabla_{\alpha} g_{\varepsilon} \mid \frac{1}{\varepsilon} \nabla_{3} g_{\varepsilon}\right)\right\|_{L^{p}\left(\Omega ; \mathbb{R}^{3 \times 3}\right)}^{p-1}\right. \\
& \left.\quad+\left\|\nabla_{\alpha} g\right\|_{L^{p}\left(\Omega ; \mathbb{R}^{3 \times 2}\right)}^{p-1}+\left\|\left(\varepsilon \nabla_{\alpha} b_{j} \mid H_{j}\right)\right\|_{L^{p}\left(\Omega ; \mathbb{R}^{3 \times 3}\right)}^{p-1}\right)\left\|\left(\nabla_{\alpha} g_{\varepsilon}-\nabla_{\alpha} g-\varepsilon \nabla_{\alpha} b_{j} \mid \frac{1}{\varepsilon} \nabla_{3} g_{\varepsilon}-H_{j}\right)\right\|_{L^{p}\left(\Omega ; \mathbb{R}^{3 \times 3}\right)} .
\end{aligned}
$$

Passing to the limit when $\varepsilon \rightarrow 0,(3.28)$ yields

$$
\limsup _{\varepsilon \rightarrow 0} \int_{\Omega} W\left(\nabla_{\alpha} u_{\varepsilon}^{j} \mid \frac{1}{\varepsilon} \nabla_{3} u_{\varepsilon}^{j}\right) d x \leq \limsup _{\varepsilon \rightarrow 0} \int_{\Omega} W\left(\nabla_{\alpha} v_{\varepsilon} \mid \frac{1}{\varepsilon} \nabla_{3} v_{\varepsilon}\right) d x+c^{\prime}\left\|H-H_{j}\right\|_{L^{p}\left(\Omega ; \mathbb{R}^{3}\right)} .
$$

Gathering (3.30), (3.31), (3.32) and remembering that $H_{j} \rightarrow H$ in $L^{p}\left(\Omega ; \mathbb{R}^{3}\right)$, we get that

$$
\mathcal{J}^{g}(u)=\lim _{j \rightarrow+\infty} \lim _{\varepsilon \rightarrow 0}\left[\int_{\Omega} W\left(\nabla_{\alpha} u_{\varepsilon}^{j} \mid \frac{1}{\varepsilon} \nabla_{3} u_{\varepsilon}^{j}\right) d x+\int_{S\left(u_{\varepsilon}^{j}\right)}\left|\left(\left(\nu_{u_{\varepsilon}^{j}}\right)_{\alpha} \mid \frac{1}{\varepsilon}\left(\nu_{u_{\varepsilon}^{j}}\right)_{3}\right)\right| d \mathcal{H}^{2}\right]
$$

where we have also used the $\Gamma$-lim inf inequality. A standard diagonalization procedure (see e.g. Lemma 7.1 in [10]) implies the existence of a sequence $j_{\varepsilon} \nearrow+\infty$ as $\varepsilon \rightarrow+\infty$ such that $u_{\varepsilon}:=u_{\varepsilon}^{j_{\varepsilon}} \in S B V^{p}\left(\Omega^{\prime} ; \mathbb{R}^{3}\right)$, $u_{\varepsilon} \rightarrow u$ in $L^{1}\left(\Omega^{\prime} ; \mathbb{R}^{3}\right), u_{\varepsilon}=g_{\varepsilon}$ on $\left[\omega^{\prime} \backslash \bar{\omega}\right] \times I$ and

$$
\mathcal{J}^{g}(u)=\lim _{\varepsilon \rightarrow 0}\left[\int_{\Omega} W\left(\nabla_{\alpha} u_{\varepsilon} \mid \frac{1}{\varepsilon} \nabla_{3} u_{\varepsilon}\right) d x+\int_{S\left(u_{\varepsilon}\right)}\left|\left(\left(\nu_{u_{\varepsilon}}\right)_{\alpha} \mid \frac{1}{\varepsilon}\left(\nu_{u_{\varepsilon}}\right)_{3}\right)\right| d \mathcal{H}^{2}\right] .
$$

If $u$ does not belong to $L^{\infty}\left(\omega^{\prime} ; \mathbb{R}^{3}\right)$, we can consider $\varphi_{i}(u) \in S B V^{p}\left(\omega^{\prime} ; \mathbb{R}^{3}\right) \cap L^{\infty}\left(\omega^{\prime} ; \mathbb{R}^{3}\right)$ where $\varphi_{i} \in \mathcal{C}_{c}^{1}\left(\mathbb{R}^{3} ; \mathbb{R}^{3}\right)$ is the truncation function defined in (3.5) and $i$ is large enough (independently of $\varepsilon$ ) so 
that $e^{i}>\|g\|_{L^{\infty}\left(\omega^{\prime} ; \mathbb{R}^{3}\right)}$. In particular, $\varphi_{i}(u)=\varphi_{i}(g)=g$ on $\omega^{\prime} \backslash \bar{\omega}$ and we can apply the previous case. It implies, for each $i \in \mathbb{N}$, the existence of a sequence $\left\{u_{\varepsilon}^{i}\right\} \subset S B V^{p}\left(\Omega^{\prime} ; \mathbb{R}^{3}\right)$ strongly converging to $\varphi_{i}(u)$ in $L^{1}\left(\Omega^{\prime} ; \mathbb{R}^{3}\right)$ satisfying $u_{\varepsilon}^{i}=g_{\varepsilon}$ on $\left[\omega^{\prime} \backslash \bar{\omega}\right] \times I$ and

$$
\mathcal{J}\left(\varphi_{i}(u)\right)=\lim _{\varepsilon \rightarrow 0} \mathcal{I}_{\varepsilon}^{g_{\varepsilon}}\left(u_{\varepsilon}^{i}\right) .
$$

Since $\varphi_{i}(u) \rightarrow u$ in $L^{1}\left(\omega^{\prime} ; \mathbb{R}^{3}\right)$ we get that

$$
\lim _{i \rightarrow+\infty} \lim _{\varepsilon \rightarrow 0}\left\|u_{\varepsilon}^{i}-u\right\|_{L^{1}\left(\Omega^{\prime} ; \mathbb{R}^{3}\right)}=0 .
$$

Furthermore, by (3.24) together with the lower semicontinuity of $\mathcal{J}$ with respect to the strong $L^{1}\left(\omega^{\prime} ; \mathbb{R}^{3}\right)$ convergence, we obtain that

$$
\mathcal{J}^{g}(u)=\lim _{i \rightarrow+\infty} \lim _{\varepsilon \rightarrow 0} \mathcal{I}_{\varepsilon}^{g_{\varepsilon}}\left(u_{\varepsilon}^{i}\right) .
$$

A standard diagonalization argument (see e.g. Lemma 7.1 in [10]) applied to (3.34) and (3.35) yields the existence of a sequence $i_{\varepsilon} \nearrow+\infty$ as $\varepsilon \rightarrow 0$ such that $u_{\varepsilon}:=u_{\varepsilon}^{i_{\varepsilon}} \in S B V^{p}\left(\Omega^{\prime}, \mathbb{R}^{3}\right), u_{\varepsilon}=g_{\varepsilon}$ on $\left[\omega^{\prime} \backslash \bar{\omega}\right] \times I$ and

$$
\mathcal{J}^{g}(u)=\lim _{\varepsilon \rightarrow 0} \mathcal{I}_{\varepsilon}^{g_{\varepsilon}}\left(u_{\varepsilon}\right)
$$

\section{A few tools}

\subsection{Convergence of sets}

The notion of $\sigma^{p}$-convergence introduced in $[13,14]$ does not seem to naturally provide a one dimensional limit crack. Indeed, let $\Gamma_{n} \subset \Omega^{\prime}$ be a sequence of $\mathcal{H}^{2}$-rectifiable sets; we denote by $\nu_{\Gamma_{n}}$ its generalized normal defined $\mathcal{H}^{2}$-a.e. on $\Gamma_{n}$. We assume that there is an a priori bound on the scaled surface energy associated with this sequence of cracks i.e.

$$
\sup _{n \in \mathbb{N}} \int_{\Gamma_{n}}\left|\left(\left(\nu_{\Gamma_{n}}\right)_{\alpha} \mid \frac{1}{\varepsilon_{n}}\left(\nu_{\Gamma_{n}}\right)_{3}\right)\right| d \mathcal{H}^{2}<+\infty
$$

Note that this bound will appear naturally in the energy estimates. Intuitively, we expect that any limit crack of $\Gamma_{n}$ will be a subset of $\omega^{\prime}$ of Hausdorff dimension equal to one. But, the sequences of test functions taken in the definition of the $\sigma^{p}$-convergence do not contain enough information in order for this to be true. Indeed, (4.1) implies in particular that $\mathcal{H}^{2}\left(\Gamma_{n}\right) \leq C$, thus according to Lemma 4.7 in [13], we have (for a subsequence) that $\Gamma_{n} \sigma^{p}$-converges in $\Omega^{\prime}$ to some $\mathcal{H}^{2}$-rectifiable set $\Gamma \subset \Omega^{\prime}$. We would like to be able to state that $\Gamma$ is of the form $\gamma \times I$ for some $\mathcal{H}^{1}$-rectifiable set $\gamma \subset \omega^{\prime}$. By lower semicontinuity of

$$
v \mapsto \int_{S(v)}\left|\left(\nu_{v}\right)_{3}\right| d \mathcal{H}^{2}
$$

for the weak $S B V^{p}\left(\Omega^{\prime}\right)$-convergence, we have, according to Lemma 4.3 in [13] and (4.1), that $\left(\nu_{\Gamma}\right)_{3}(x)=0$ $\mathcal{H}^{2}$-a.e. $x \in \Gamma$. But this does not tell us that $\Gamma \cong \gamma \times I$. We know, by the very definition of the $\sigma^{p}$ convergence, that there exists a function $u \in S B V^{p}\left(\Omega^{\prime}\right)$ and a sequence $u_{n} \rightarrow u$ in $S B V^{p}\left(\Omega^{\prime}\right)$ such that $S\left(u_{n}\right) \widetilde{\subset} \Gamma_{n}$ and $\Gamma \cong S(u)$. To prove that $\Gamma \cong \gamma \times I$, it would be enough to show that $D_{3} u=0$ in the sense of Radon measures. This would be immediate if the approximate scaled gradient of $u_{n}$ was bounded in $L^{p}\left(\Omega^{\prime} ; \mathbb{R}^{3}\right)$. Since, in the sequel, we will only be interested in minimizing sequences satisfying this property, it prompts us to redefine the notion of $\sigma^{p}$-convergence in a 3D-2D dimensional reduction setting. 
QUASISTATIC EVOLUTION OF A BRITTLE THIN FILM

Definition 4.1. Let $\left\{\varepsilon_{n}\right\} \searrow 0^{+}$and $\Gamma_{n} \subset \Omega^{\prime}$ be a sequence of $\mathcal{H}^{2}$-rectifiable sets. We say that $\Gamma_{n}$ converges towards $\gamma$ in $\Omega^{\prime}$ if $\gamma \subset \omega^{\prime},(4.1)$ holds and

(a) if $u_{k} \rightarrow u$ in $S B V^{p}\left(\Omega^{\prime}\right), S\left(u_{k}\right) \widetilde{\subset} \Gamma_{n_{k}}$ and

$$
\sup _{k \in \mathbb{N}} \int_{\Omega^{\prime}}\left|\left(\nabla_{\alpha} u_{k} \mid \frac{1}{\varepsilon_{n_{k}}} \nabla_{3} u_{k}\right)\right|^{p} d x<+\infty
$$

for a subsequence $\left\{\varepsilon_{n_{k}}\right\} \subset\left\{\varepsilon_{n}\right\}$, then $u \in S B V^{p}\left(\omega^{\prime}\right)$ and $S(u) \widetilde{\subset} \gamma$;

(b) there exists a function $u \in S B V^{p}\left(\omega^{\prime}\right)$ and a sequence $u_{n} \in S B V^{p}\left(\Omega^{\prime}\right)$ such that $u_{n} \rightarrow u$ in $S B V^{p}\left(\Omega^{\prime}\right), S\left(u_{n}\right) \widetilde{\subset} \Gamma_{n}$,

$$
\sup _{n \in \mathbb{N}} \int_{\Omega^{\prime}}\left|\left(\nabla_{\alpha} u_{n} \mid \frac{1}{\varepsilon_{n}} \nabla_{3} u_{n}\right)\right|^{p} d x<+\infty
$$

and $S(u) \cong \gamma$.

According to property (b) of Definition 4.1, $\gamma$ is necessarily a $\mathcal{H}^{1}$-rectifiable set. In the following Remark, we state few properties of this kind of convergence as lower semicontinuity with respect to the Hausdorff measure and stability with respect to the inclusion.

Remark 4.2. Let $\Gamma_{n} \rightarrow \gamma$ in the sense of Definition 4.1, then

1. for every Borel set $E \subset \omega^{\prime}$ such that $\mathcal{H}^{1}(E)<+\infty$ (or $E$ a compact set),

$$
2 \mathcal{H}^{1}(\gamma \backslash E) \leq \liminf _{n \rightarrow+\infty} \mathcal{H}^{2}\left(\Gamma_{n} \backslash(E \times[-1,1])\right) ;
$$

2. if $\Gamma_{n} \widetilde{\subset} \Gamma_{n}^{\prime}$ and $\Gamma_{n}^{\prime} \rightarrow \gamma^{\prime}$ in the sense of Definition 4.1, then $\gamma \widetilde{\subset} \gamma^{\prime}$;

3. if $\Gamma_{n} \stackrel{\sigma^{p}}{\longrightarrow} \Gamma$, then $\gamma \times I \widetilde{\subset} \Gamma$.

Replacing every approximate gradients by approximate scaled gradients and using Lemma 2.2 instead of Ambrosio's Compactness Theorem, the exact analogues of the proofs of Lemma 4.5 and Proposition 4.6 in [13] would demonstrate that any sequence of $\mathcal{H}^{2}$-rectifiable sets $\Gamma_{n} \subset \Omega^{\prime}$ satisfying (4.1) admits a convergent subsequence in the sense of Definition (4.1). But this compactness result will not be sufficient because, in the proof of Theorem 2.1, we will deal with sequence of $\mathcal{H}^{2}$-rectifiable sets which are increasing with respect to the time parameter $t$. The following Proposition, which is the analogue of Lemma 4.8 in [13], states a version of Helly's Theorem for a sequence of increasing $\mathcal{H}^{2}$-rectifiable sets.

Proposition 4.3. Let $[0, T] \ni t \mapsto \Gamma_{n}(t)$ a sequence of $\mathcal{H}^{2}$-rectifiable sets of $\Omega^{\prime}$ that increases with $t$, i.e.

$$
\Gamma_{n}(s) \widetilde{\subset} \Gamma_{n}(t) \subset \Omega^{\prime}, \quad \text { for every } s, t \in[0, T] \text { with } s<t .
$$

Assume that

$$
\sup _{n \in \mathbb{N}} \int_{\Gamma_{n}(t)}\left|\left(\left(\nu_{\Gamma_{n}(t)}\right)_{\alpha} \mid \frac{1}{\varepsilon_{n}}\left(\nu_{\Gamma_{n}(t)}\right)_{3}\right)\right| d \mathcal{H}^{2}<+\infty
$$

uniformly in $t$. Then, there exists a subsequence $\Gamma_{n_{k}}(t)$ and a t-increasing $\mathcal{H}^{1}$-rectifiable set $\gamma(t) \subset \omega^{\prime}$ such that for every $t \in[0, T], \Gamma_{n_{k}}(t)$ converges to $\gamma(t)$ in the sense of Definition 4.1. 


\subsection{Transfer of jump sets}

We now state a Jump Transfer theorem in a rescaled version. It permits, under weak $S B V^{p}\left(\Omega^{\prime} ; \mathbb{R}^{3}\right)$ convergence assumptions of a sequence $\left\{u_{n}\right\}$ - with associated bounded scaled bulk energy - toward its limit $u$, the transfer of the part of the jump set of a $2 \mathrm{D}$ admissible deformation that lies in the jump set of $u$ onto that of the sequence $\left\{u_{n}\right\}$. The proof relies on De Giorgi's slicing argument.

Theorem 4.4 (Jump Transfer). Let $\left\{u_{n}\right\} \subset S B V^{p}\left(\Omega^{\prime} ; \mathbb{R}^{3}\right)$ and $u \in S B V^{p}\left(\omega^{\prime} ; \mathbb{R}^{3}\right)$ such that $S\left(u_{n}\right) \subset$ $\bar{\omega} \times I, u_{n} \rightarrow u$ in $L^{1}\left(\Omega^{\prime} ; \mathbb{R}^{3}\right)$ and

$$
M:=\sup _{n \in \mathbb{N}} \int_{\Omega^{\prime}}\left|\left(\nabla_{\alpha} u_{n} \mid \frac{1}{\varepsilon_{n}} \nabla_{3} u_{n}\right)\right|^{p} d x<+\infty .
$$

Then, for all $\phi \in S B V^{p}\left(\omega^{\prime} ; \mathbb{R}^{3}\right)$, there exists $\left\{\phi_{n}\right\} \subset S B V^{p}\left(\Omega^{\prime} ; \mathbb{R}^{3}\right)$ such that

- $\phi_{n}=\phi$ a.e. on $\left[\omega^{\prime} \backslash \bar{\omega}\right] \times I$,

- $\phi_{n} \rightarrow \phi$ in $L^{1}\left(\Omega^{\prime} ; \mathbb{R}^{3}\right)$,

- $\left(\nabla_{\alpha} \phi_{n} \mid \frac{1}{\varepsilon_{n}} \nabla_{3} \phi_{n}\right) \rightarrow\left(\nabla_{\alpha} \phi \mid 0\right)$ in $L^{p}\left(\Omega^{\prime} ; \mathbb{R}^{3 \times 3}\right)$,

- $\int_{\left.\left[S\left(\phi_{n}\right) \backslash S\left(u_{n}\right)\right] \backslash S(\phi) \backslash S(u)\right]}\left|\left(\left(\nu_{\phi_{n}}\right)_{\alpha} \mid \frac{1}{\varepsilon_{n}}\left(\nu_{\phi_{n}}\right)_{3}\right)\right| d \mathcal{H}^{2} \rightarrow 0$.

Proof. We first undo the scaling, coming back to the cylinder of thickness $2 \varepsilon_{n}$. Then, we extend periodically the function in the transverse direction. Note that the periodic extension may generate some additional jump at the interface of each slice of thickness $2 \varepsilon_{n}$. Despite this new discontinuities, we can still apply the classical Jump Transfer Theorem (Theorem 2.1 in [18]) and, by contradiction, we show that we can choose a slice of thickness $2 \varepsilon_{n}$ that satisfies good estimations. Finally, we observe that, after translation and dilation, the restriction of the function to this particular slice satisfies the conclusion of Theorem 4.4.

Step 1. We come back to the non rescaled cylinder $\Omega_{\varepsilon_{n}}^{\prime}$ of thickness $2 \varepsilon_{n}$. We set $v_{n}\left(x_{\alpha}, x_{3}\right):=$ $u_{n}\left(x_{\alpha}, x_{3} / \varepsilon_{n}\right)$. Thus $v_{n} \in S B V^{p}\left(\Omega_{\varepsilon_{n}}^{\prime} ; \mathbb{R}^{3}\right)$ and $S\left(v_{n}\right) \subset \bar{\omega} \times\left(-\varepsilon_{n}, \varepsilon_{n}\right)$. Moreover,

$$
\left\{\begin{array}{l}
\frac{1}{\varepsilon_{n}} \int_{\Omega_{\varepsilon_{n}^{\prime}}}\left|v_{n}-u\right| d x=\int_{\Omega^{\prime}}\left|u_{n}-u\right| d x, \\
\frac{1}{\varepsilon_{n}} \int_{\Omega_{\varepsilon_{n}}^{\prime}}\left|\nabla v_{n}\right|^{p} d x=\int_{\Omega^{\prime}}\left|\left(\nabla_{\alpha} u_{n} \mid \frac{1}{\varepsilon_{n}} \nabla_{3} u_{n}\right)\right|^{p} d x .
\end{array}\right.
$$

We now to extend $v_{n}$ by periodicity in the $x_{3}$ direction. The discontinuities of the resulting function will be those inherited from the discontinuities of $v_{n}$ and from additional jumps that may occur at the interface of each slice. Let

$$
N_{n}:= \begin{cases}\frac{1}{2 \varepsilon_{n}}-\frac{1}{2} & \text { if } \frac{1}{2 \varepsilon_{n}}+\frac{1}{2} \in \mathbb{N}, \\ \llbracket \frac{1}{2 \varepsilon_{n}}+\frac{1}{2} \rrbracket & \text { otherwise. }\end{cases}
$$

For every $i \in\left\{-N_{n}, \ldots, N_{n}\right\}$, we set $I_{i, n}:=\left((2 i-1) \varepsilon_{n},(2 i+1) \varepsilon_{n}\right)$ and $\Omega_{i, n}^{\prime}:=\omega^{\prime} \times I_{i, n}$. Note that $N_{n}$ is the smaller integer such that $\Omega^{\prime} \cap \Omega_{i, n}^{\prime} \neq \emptyset$ for every $i \in\left\{-N_{n}, \ldots, N_{n}\right\}$. We define the function $w_{n}$ on $\Omega^{\prime}(n):=\omega^{\prime} \times\left(-\left(2 N_{n}+1\right) \varepsilon_{n},\left(2 N_{n}+1\right) \varepsilon_{n}\right)$ by extending $v_{n}$ by periodicity in the $x_{3}$ direction on $\Omega^{\prime}(n)$ :

$$
w_{n}\left(x_{\alpha}, x_{3}\right)=v_{n}\left(x_{\alpha}, x_{3}-2 i \varepsilon_{n}\right) \text { if } x_{3} \in I_{i, n} .
$$


Since $\Omega^{\prime} \subset \Omega^{\prime}(n), w_{n}$ is a fortiori defined on $\Omega^{\prime}, w_{n} \in S B V^{p}\left(\Omega^{\prime} ; \mathbb{R}^{3}\right)$ and $S\left(w_{n}\right) \cap \Omega^{\prime} \subset \bar{\omega} \times I$.

Step 2. We would like to apply the classical Jump Transfer Theorem (Theorem 2.1 in [18]) to the function $w_{n}$. From (4.2), we have that

$$
\begin{aligned}
\int_{\Omega^{\prime}}\left|w_{n}-u\right| d x & =\sum_{i=-N_{n}}^{N_{n}} \int_{\Omega_{i, n}^{\prime} \cap \Omega^{\prime}}\left|v_{n}\left(x_{\alpha}, x_{3}-2 i \varepsilon_{n}\right)-u\left(x_{\alpha}\right)\right| d x \\
& \leq\left(2 N_{n}+1\right) \int_{\Omega_{\varepsilon_{n}}^{\prime}}\left|v_{n}-u\right| d x \\
& =\varepsilon_{n}\left(2 N_{n}+1\right) \int_{\Omega^{\prime}}\left|u_{n}-u\right| d x \\
& \leq\left(1+2 \varepsilon_{n}\right) \int_{\Omega^{\prime}}\left|u_{n}-u\right| d x \rightarrow 0
\end{aligned}
$$

and

$$
\begin{aligned}
\int_{\Omega^{\prime}}\left|\nabla w_{n}\right|^{p} d x & =\sum_{i=-N_{n}}^{N_{n}} \int_{\Omega_{i, n}^{\prime} \cap \Omega^{\prime}}\left|\nabla v_{n}\left(x_{\alpha}, x_{3}-2 i \varepsilon_{n}\right)\right|^{p} d x \\
& \leq\left(2 N_{n}+1\right) \int_{\Omega_{\varepsilon_{n}}^{\prime}}\left|\nabla v_{n}\right|^{p} d x \\
& =\varepsilon_{n}\left(2 N_{n}+1\right) \int_{\Omega^{\prime}}\left|\left(\nabla_{\alpha} u_{n} \mid \frac{1}{\varepsilon_{n}} \nabla_{3} u_{n}\right)\right|^{p} d x \\
& \leq\left(1+2 \varepsilon_{n}\right) M,
\end{aligned}
$$

which implies, thanks to De La Vallée Poussin criterion (see Proposition 1.27 in [2]), that the sequence $\left\{\left|\nabla w_{n}\right|\right\}$ is equi-integrable. We are now in position to apply Theorem 2.1 in [18] to the sequence $\left\{w_{n}\right\}$. Indeed, an inspection of the proof of this result shows that the weak $L^{1}$-convergence required by $\left\{\left|\nabla w_{n}\right|\right\}$ can be replaced, without passing to a subsequence, by its equi-integrability (see p. 1477 in [18]). Thus, for all $\phi \in S B V^{p}\left(\omega^{\prime} ; \mathbb{R}^{3}\right)$, we get the existence of a sequence $\left\{\psi_{n}\right\} \subset S B V^{p}\left(\Omega^{\prime} ; \mathbb{R}^{3}\right)$ such that

- $\psi_{n}=\phi$ a.e. on $\left[\omega^{\prime} \backslash \bar{\omega}\right] \times I$,

- $\psi_{n} \rightarrow \phi$ in $L^{1}\left(\Omega^{\prime} ; \mathbb{R}^{3}\right)$,

- $\nabla \psi_{n} \rightarrow\left(\nabla_{\alpha} \phi \mid 0\right)$ in $L^{p}\left(\Omega^{\prime} ; \mathbb{R}^{3 \times 3}\right)$,

- $\mathcal{H}^{2}\left(\left[S\left(\psi_{n}\right) \backslash S\left(w_{n}\right)\right] \backslash[S(\phi) \backslash S(u)]\right) \rightarrow 0$.

Step 3. Since $\bigcup_{i=-N_{n}+1}^{N_{n}-1} \Omega_{i, n}^{\prime} \subset \Omega^{\prime}$, we may find a $i_{n} \in\left\{-N_{n}+1, \ldots, N_{n}-1\right\}$ such that

$$
\begin{array}{r}
\left(2 N_{n}-1\right)\left\{\int_{\Omega_{i_{n}, n}^{\prime}}\left|\psi_{n}-\phi\right| d x+\int_{\Omega_{i_{n}, n}^{\prime}}\left|\nabla \psi_{n}-\left(\nabla_{\alpha} \phi \mid 0\right)\right|^{p} d x\right. \\
\left.+\mathcal{H}_{\left\lfloor\Omega_{i_{n}, n}^{\prime}\right.}^{2}\left(\left[S\left(\psi_{n}\right) \backslash S\left(w_{n}\right)\right] \backslash[S(\phi) \backslash S(u)]\right)\right\} \\
\leq \int_{\Omega^{\prime}}\left|\psi_{n}-\phi\right| d x+\int_{\Omega^{\prime}}\left|\nabla \psi_{n}-\left(\nabla_{\alpha} \phi \mid 0\right)\right|^{p} d x+\mathcal{H}^{2}\left(\left[S\left(\psi_{n}\right) \backslash S\left(w_{n}\right)\right] \backslash[S(\phi) \backslash S(u)]\right) .
\end{array}
$$




\section{JEAN-FranÇOIS BABADJIAN}

Since $2 N_{n}-1 \geq 1 / \varepsilon_{n}-2$, we have

$$
\begin{array}{r}
\frac{1}{\varepsilon_{n}}\left\{\int_{\Omega_{i_{n}, n}^{\prime}}\left|\psi_{n}-\phi\right| d x+\int_{\Omega_{i_{n}, n}^{\prime}}\left|\nabla \psi_{n}-\left(\nabla_{\alpha} \phi \mid 0\right)\right|^{p} d x+\mathcal{H}_{\left\lfloor\Omega_{i_{n}, n}^{\prime}\right.}^{2}\left(\left[S\left(\psi_{n}\right) \backslash S\left(w_{n}\right)\right] \backslash[S(\phi) \backslash S(u)]\right)\right\} \\
\leq 3 \int_{\Omega^{\prime}}\left|\psi_{n}-\phi\right| d x+3 \int_{\Omega^{\prime}}\left|\nabla \psi_{n}-\left(\nabla_{\alpha} \phi \mid 0\right)\right|^{p} d x+3 \mathcal{H}^{2}\left(\left[S\left(\psi_{n}\right) \backslash S\left(w_{n}\right)\right] \backslash[S(\phi) \backslash S(u)]\right) .
\end{array}
$$

Step 4. We will show that, after a translation and a dilation, $\psi_{n\left\lfloor\Omega_{i_{n}, n}^{\prime}\right.}$ is the right candidate for Theorem 4.4. Let us come back to the cylinder $\Omega_{\varepsilon_{n}}^{\prime}=\omega^{\prime} \times\left(-\varepsilon_{n}, \varepsilon_{n}\right)$; letting

$$
\varphi_{n}\left(x_{\alpha}, x_{3}\right):=\psi_{n\left\lfloor\Omega_{i_{n}, n}^{\prime}\right.}\left(x_{\alpha}, x_{3}+2 i_{n} \varepsilon_{n}\right) \text { if } x_{3} \in\left(-\varepsilon_{n}, \varepsilon_{n}\right),
$$

then $\varphi_{n} \in S B V^{p}\left(\Omega_{\varepsilon_{n}}^{\prime} ; \mathbb{R}^{3}\right), \varphi_{n}=\phi$ a.e. on $\left[\omega^{\prime} \backslash \bar{\omega}\right] \times\left(-\varepsilon_{n}, \varepsilon_{n}\right)$ and

$$
\begin{aligned}
& \frac{1}{\varepsilon_{n}}\left\{\int_{\Omega_{\varepsilon_{n}}^{\prime}}\left|\varphi_{n}-\phi\right| d x+\int_{\Omega_{\varepsilon_{n}}^{\prime}}\left|\nabla \varphi_{n}-\left(\nabla_{\alpha} \phi \mid 0\right)\right|^{p} d x+\mathcal{H}_{\left[\Omega_{\varepsilon_{n}}^{\prime}\right.}^{2}\left(\left[S\left(\varphi_{n}\right) \backslash S\left(v_{n}\right)\right] \backslash[S(\phi) \backslash S(u)]\right)\right\} \\
& \quad \leq 3 \int_{\Omega^{\prime}}\left|\psi_{n}-\phi\right| d x+3 \int_{\Omega^{\prime}}\left|\nabla \psi_{n}-\left(\nabla_{\alpha} \phi \mid 0\right)\right|^{p} d x+3 \mathcal{H}^{2}\left(\left[S\left(\psi_{n}\right) \backslash S\left(w_{n}\right)\right] \backslash[S(\phi) \backslash S(u)]\right) .
\end{aligned}
$$

Performing the scaling so as to come back to the unit cylinder, we get, upon setting $\phi_{n}\left(x_{\alpha}, x_{3}\right):=$ $\varphi_{n}\left(x_{\alpha}, \varepsilon_{n} x_{3}\right)$, that $\phi_{n} \in S B V^{p}\left(\Omega^{\prime} ; \mathbb{R}^{3}\right), \phi_{n}=\phi$ a.e. on $\left[\omega^{\prime} \backslash \bar{\omega}\right] \times I$ and

$$
\begin{aligned}
\int_{\Omega^{\prime}}\left|\phi_{n}-\phi\right| d x+\int_{\Omega^{\prime}}\left|\left(\nabla_{\alpha} \phi_{n} \mid \frac{1}{\varepsilon_{n}} \nabla_{3} \phi_{n}\right)-\left(\nabla_{\alpha} \phi \mid 0\right)\right|^{p} d x \\
+\int_{\left[S\left(\phi_{n}\right) \backslash S\left(u_{n}\right)\right] \backslash[S(\phi) \backslash S(u)]}\left|\left(\left(\nu_{\phi_{n}}\right)_{\alpha} \mid \frac{1}{\varepsilon_{n}}\left(\nu_{\phi_{n}}\right)_{3}\right)\right| d \mathcal{H}^{2} \\
\leq 3 \int_{\Omega^{\prime}}\left|\psi_{n}-\phi\right| d x+3 \int_{\Omega^{\prime}}\left|\nabla \psi_{n}-\left(\nabla_{\alpha} \phi \mid 0\right)\right|^{p} d x \\
+3 \mathcal{H}^{2}\left(\left[S\left(\psi_{n}\right) \backslash S\left(w_{n}\right)\right] \backslash[S(\phi) \backslash S(u)]\right) \rightarrow 0 .
\end{aligned}
$$

Remark 4.5. Since for $\mathcal{H}^{2}$-a.e. $x \in S\left(\phi_{n}\right) \cap S(\phi), \nu_{\phi_{n}}(x)= \pm \nu_{\phi}(x)$, we have

$$
\begin{gathered}
\int_{\left.\left[S\left(\phi_{n}\right) \backslash S\left(u_{n}\right)\right] \backslash S(\phi) \backslash S(u)\right]}\left|\left(\left(\nu_{\phi_{n}}\right)_{\alpha} \mid \frac{1}{\varepsilon_{n}}\left(\nu_{\phi_{n}}\right)_{3}\right)\right| d \mathcal{H}^{2} \\
\quad \geq \int_{S\left(\phi_{n}\right) \backslash S\left(u_{n}\right)}\left|\left(\left(\nu_{\phi_{n}}\right)_{\alpha} \mid \frac{1}{\varepsilon_{n}}\left(\nu_{\phi_{n}}\right)_{3}\right)\right| d \mathcal{H}^{2}-\int_{S(\phi) \backslash S(u)}\left|\left(\left(\nu_{\phi}\right)_{\alpha} \mid 0\right)\right| d \mathcal{H}^{2} \\
=\int_{S\left(\phi_{n}\right) \backslash S\left(u_{n}\right)}\left|\left(\left(\nu_{\phi_{n}}\right)_{\alpha} \mid \frac{1}{\varepsilon_{n}}\left(\nu_{\phi_{n}}\right)_{3}\right)\right| d \mathcal{H}^{2}-2 \mathcal{H}^{1}(S(\phi) \backslash S(u)),
\end{gathered}
$$

thus

$$
\limsup _{n \rightarrow+\infty} \int_{S\left(\phi_{n}\right) \backslash S\left(u_{n}\right)}\left|\left(\left(\nu_{\phi_{n}}\right)_{\alpha} \mid \frac{1}{\varepsilon_{n}}\left(\nu_{\phi_{n}}\right)_{3}\right)\right| d \mathcal{H}^{2} \leq 2 \mathcal{H}^{1}(S(\phi) \backslash S(u)) .
$$

The following Theorem establishes a link between the convergence in the sense of Definition 4.1 and the Jump Transfer Theorem. It will allow to pass to the limit in the surface energy.

Theorem 4.6. Let $\Gamma_{n} \subset \Omega^{\prime}$ be a sequence of $\mathcal{H}^{2}$-rectifiable sets converging towards $\gamma$ in the sense of Definition 4.1. Then, for every $v \in S B V^{p}\left(\omega^{\prime} ; \mathbb{R}^{3}\right)$, there exists $\left\{v_{n}\right\} \subset S B V^{p}\left(\Omega^{\prime} ; \mathbb{R}^{3}\right)$ such that $v_{n}=v$ a.e. on $\left[\omega^{\prime} \backslash \bar{\omega}\right] \times I$, 
- $v_{n} \rightarrow v$ in $L^{1}\left(\Omega^{\prime} ; \mathbb{R}^{3}\right)$,

- $\left(\nabla_{\alpha} v_{n} \mid \frac{1}{\varepsilon_{n}} \nabla_{3} v_{n}\right) \rightarrow\left(\nabla_{\alpha} v \mid 0\right)$ in $L^{p}\left(\Omega^{\prime} ; \mathbb{R}^{3 \times 3}\right)$,

- $\limsup _{n \rightarrow+\infty} \int_{S\left(v_{n}\right) \backslash \Gamma_{n}}\left|\left(\left(\nu_{v_{n}}\right)_{\alpha} \mid \frac{1}{\varepsilon_{n}}\left(\nu_{v_{n}}\right)_{3}\right)\right| d \mathcal{H}^{2} \leq 2 \mathcal{H}^{1}(S(v) \backslash \gamma)$.

Proof. According to Definition 4.1 (b), there exists a function $u \in S B V^{p}\left(\omega^{\prime} ; \mathbb{R}^{3}\right)$ and a sequence $\left\{u_{n}\right\} \subset$ $S B V^{p}\left(\Omega^{\prime} ; \mathbb{R}^{3}\right)$ such that $u_{n} \rightarrow u$ in $S B V^{p}\left(\Omega^{\prime} ; \mathbb{R}^{3}\right), S\left(u_{n}\right) \widetilde{\subset} \Gamma_{n}, S(u) \cong \gamma$ and

$$
\sup _{n \in \mathbb{N}} \int_{\Omega^{\prime}}\left|\left(\nabla_{\alpha} u_{n} \mid \frac{1}{\varepsilon_{n}} \nabla_{3} u_{n}\right)\right|^{p} d x<+\infty \text {. }
$$

Theorem 4.4 and Remark 4.5 yield, for any $v \in S B V^{p}\left(\omega^{\prime} ; \mathbb{R}^{3}\right)$, the existence of a sequence $\left\{v_{n}\right\} \subset$ $S B V^{p}\left(\Omega^{\prime} ; \mathbb{R}^{3}\right)$ such that $v_{n}=v$ a.e. on $\left[\omega^{\prime} \backslash \bar{\omega}\right] \times I$,

- $v_{n} \rightarrow v$ in $L^{1}\left(\Omega^{\prime} ; \mathbb{R}^{3}\right)$,

- $\left(\nabla_{\alpha} v_{n} \mid \frac{1}{\varepsilon_{n}} \nabla_{3} v_{n}\right) \rightarrow\left(\nabla_{\alpha} v \mid 0\right)$ in $L^{p}\left(\Omega^{\prime} ; \mathbb{R}^{3 \times 3}\right)$,

- $\limsup _{n \rightarrow+\infty} \int_{S\left(v_{n}\right) \backslash S\left(u_{n}\right)}\left|\left(\left(\nu_{v_{n}}\right)_{\alpha} \mid \frac{1}{\varepsilon_{n}}\left(\nu_{v_{n}}\right)_{3}\right)\right| d \mathcal{H}^{2} \leq 2 \mathcal{H}^{1}(S(v) \backslash S(u))$.

As $S\left(u_{n}\right) \widetilde{\subset} \Gamma_{n}$ and $S(u) \cong \gamma$, we get

$$
\limsup _{n \rightarrow+\infty} \int_{S\left(v_{n}\right) \backslash \Gamma_{n}}\left|\left(\left(\nu_{v_{n}}\right)_{\alpha} \mid \frac{1}{\varepsilon_{n}}\left(\nu_{v_{n}}\right)_{3}\right)\right| d \mathcal{H}^{2} \leq 2 \mathcal{H}^{1}(S(v) \backslash \gamma) .
$$

\subsection{Convergence of the stresses}

The energy conservation involves the derivative of the stored energy density. Thus, we have to ensure that the $\mathcal{C}^{1}$ character of $W$ is preserved by passing to the $\Gamma$-limit. The following Proposition provides an answer to this question. For an alternative proof of that result, we refer to [3] Chapter 4.

Proposition 4.7. Let $W: \mathbb{R}^{3 \times 3} \rightarrow \mathbb{R}$ be a $\mathcal{C}^{1}$ function satisfying (2.1), then the function $\mathcal{Q} W_{0}: \mathbb{R}^{3 \times 2} \rightarrow$ $\mathbb{R}$ is of class $\mathcal{C}^{1}$.

Proof. According to [22], the function $W_{0}$ is continuous and satisfies

$$
\frac{1}{\beta}|\bar{\xi}|^{p}-\beta \leq W_{0}(\bar{\xi}) \leq \beta\left(1+|\bar{\xi}|^{p}\right)
$$

for every $\bar{\xi} \in \mathbb{R}^{3 \times 2}$. As a consequence, since $p-1>0$,

$$
\liminf _{|\bar{\xi}| \rightarrow+\infty} \frac{W_{0}(\bar{\xi})}{|\bar{\xi}|^{p-1}}=+\infty \quad \text { and } \quad \limsup _{|\bar{\xi}| \rightarrow+\infty} \frac{W_{0}(\bar{\xi})}{|\bar{\xi}|^{p}}=\beta<+\infty .
$$

Furthermore, for all $\bar{\xi} \in \mathbb{R}^{3 \times 2}$ there exists $\xi_{3} \in \mathbb{R}^{3}$ such that $W_{0}(\bar{\xi})=W\left(\bar{\xi} \mid \xi_{3}\right)$. Since $W$ is differentiable,

$$
\limsup _{|\bar{\eta}| \rightarrow 0} \frac{W_{0}(\bar{\xi}+\bar{\eta})-W_{0}(\bar{\xi})-d \cdot \bar{\eta}}{|\bar{\eta}|} \leq \limsup _{|\bar{\eta}| \rightarrow 0} \frac{W\left(\left(\bar{\xi} \mid \xi_{3}\right)+(\bar{\eta} \mid 0)\right)-W\left(\bar{\xi} \mid \xi_{3}\right)-\partial W\left(\bar{\xi} \mid \xi_{3}\right) \cdot(\bar{\eta} \mid 0)}{|(\bar{\eta} \mid 0)|}=0
$$




\section{JEAN-FranÇOIS BABADJIAN}

where $d \in \mathbb{R}^{3 \times 2}$ is defined by $d_{i j}:=\left(\partial W\left(\bar{\xi} \mid \xi_{3}\right)\right)_{i j}$ for all $i \in\{1,2,3\}$ and all $j \in\{1,2\}$. It yields that $W_{0}$ is upper semidifferentiable and the thesis follows from Theorem B in [4].

From the previous Lemma, the function $\mathcal{Q} W_{0}$ is of class $\mathcal{C}^{1}$ and we denote by $\partial\left(\mathcal{Q} W_{0}\right)$ its differential. The following result is the analogue of Lemma 4.11 in [13] in a 3D-2D dimensional reduction setting. It asserts, under assumptions of weak $S B V^{p}\left(\Omega ; \mathbb{R}^{3}\right)$-convergence of the deformations together with the convergence of the bulk energy, the weak $L^{p^{\prime}}\left(\Omega ; \mathbb{R}^{3 \times 3}\right)$-convergence the stresses.

Lemma 4.8. Let $\left\{u_{n}\right\} \subset S B V^{p}\left(\Omega ; \mathbb{R}^{3}\right)$ and $u \in S B V^{p}\left(\omega ; \mathbb{R}^{3}\right)$ such that $u_{n} \rightarrow u$ in $S B V^{p}\left(\Omega ; \mathbb{R}^{3}\right)$ and

$$
\int_{\Omega} W\left(\nabla_{\alpha} u_{n} \mid \frac{1}{\varepsilon_{n}} \nabla_{3} u_{n}\right) d x \rightarrow 2 \int_{\omega} \mathcal{Q} W_{0}\left(\nabla_{\alpha} u\right) d x_{\alpha} .
$$

Then,

$$
\partial W\left(\nabla_{\alpha} u_{n} \mid \frac{1}{\varepsilon_{n}} \nabla_{3} u_{n}\right) \rightarrow\left(\partial\left(\mathcal{Q} W_{0}\right)\left(\nabla_{\alpha} u\right) \mid 0\right) \quad \text { in } L^{p^{\prime}}\left(\Omega ; \mathbb{R}^{3 \times 3}\right) .
$$

Proof. Let $\Psi \in L^{p}\left(\Omega ; \mathbb{R}^{3 \times 3}\right)$, we denote by $\bar{\Psi} \in L^{p}\left(\Omega ; \mathbb{R}^{3 \times 2}\right)$ the restriction of $\Psi$ to $\mathbb{R}^{3 \times 2}$ i.e. $\bar{\Psi}_{i j}=\Psi_{i j}$ if $i \in\{1,2,3\}$ and $j \in\{1,2\}$. It is enough to show that

$$
\begin{aligned}
\int_{\Omega} \partial\left(\mathcal{Q} W_{0}\right)\left(\nabla_{\alpha} u\right) \cdot \bar{\Psi} d x & =\int_{\Omega}\left(\partial\left(\mathcal{Q} W_{0}\right)\left(\nabla_{\alpha} u\right) \mid 0\right) \cdot \Psi d x \\
& \leq \liminf _{n \rightarrow+\infty} \int_{\Omega} \partial W\left(\nabla_{\alpha} u_{n} \mid \frac{1}{\varepsilon_{n}} \nabla_{3} u_{n}\right) \cdot \Psi d x .
\end{aligned}
$$

Let $h_{k} \searrow 0^{+}$, according to Remark 3.2, Theorem 5.29 in [2] we have

$$
\begin{aligned}
\int_{\Omega} \mathcal{Q} W_{0}\left(\nabla_{\alpha} u+h_{k} \bar{\Psi}\right) d x & \leq \liminf _{n \rightarrow+\infty} \int_{\Omega} \mathcal{Q} W_{0}\left(\nabla_{\alpha} u_{n}+h_{k} \bar{\Psi}\right) d x \\
& \leq \liminf _{n \rightarrow+\infty} \int_{\Omega} W\left(\left(\nabla_{\alpha} u_{n} \mid \frac{1}{\varepsilon_{n}} \nabla_{3} u_{n}\right)+h_{k} \Psi\right) d x .
\end{aligned}
$$

As a consequence, from (4.3) we get that

$$
\begin{aligned}
\int_{\Omega} \frac{\mathcal{Q} W_{0}\left(\nabla_{\alpha} u+h_{k} \bar{\Psi}\right)-\mathcal{Q} W_{0}\left(\nabla_{\alpha} u\right)}{h_{k}} d x \\
\quad \leq \liminf _{n \rightarrow+\infty} \int_{\Omega} \frac{1}{h_{k}}\left[W\left(\left(\nabla_{\alpha} u_{n} \mid \frac{1}{\varepsilon_{n}} \nabla_{3} u_{n}\right)+h_{k} \Psi\right)-W\left(\nabla_{\alpha} u_{n} \mid \frac{1}{\varepsilon_{n}} \nabla_{3} u_{n}\right)\right] d x .
\end{aligned}
$$

We may find a $n_{k} \in \mathbb{N}$ such that

$$
\begin{aligned}
\int_{\Omega} \frac{\mathcal{Q} W_{0}\left(\nabla_{\alpha} u\right.}{\left.h_{k} \bar{\Psi}\right)-\mathcal{Q} W_{0}\left(\nabla_{\alpha} u\right)} & d x-\frac{1}{k} \\
h_{k} & \leq \int_{\Omega} \frac{1}{h_{k}}\left[W\left(\left(\nabla_{\alpha} u_{n} \mid \frac{1}{\varepsilon_{n}} \nabla_{3} u_{n}\right)+h_{k} \Psi\right)-W\left(\nabla_{\alpha} u_{n} \mid \frac{1}{\varepsilon_{n}} \nabla_{3} u_{n}\right)\right] d x
\end{aligned}
$$

for all $n \geq n_{k}$. We define $\eta_{n}=h_{k}$ if $n_{k} \leq n \leq n_{k+1}$ and pass to the limit when $n \rightarrow+\infty$. Since $\Phi \mapsto \int_{\Omega} W(\Phi) d x$ is a $\mathcal{C}^{1}$-map from $L^{p}\left(\Omega ; \mathbb{R}^{3 \times 3}\right)$ to $\mathbb{R}$ with differential $\Psi \mapsto \int_{\Omega} \partial W(\Phi) \cdot \Psi d x$, it follows 
that

$$
\begin{aligned}
\lim _{n \rightarrow+\infty} \int_{\Omega} \frac{\mathcal{Q} W_{0}\left(\nabla_{\alpha} u+\eta_{n} \bar{\Psi}\right)-\mathcal{Q} W_{0}\left(\nabla_{\alpha} u\right)}{\eta_{n}} d x & \\
& \leq \liminf _{n \rightarrow+\infty} \int_{\Omega} \frac{1}{\eta_{n}}\left[W\left(\left(\nabla_{\alpha} u_{n} \mid \frac{1}{\varepsilon_{n}} \nabla_{3} u_{n}\right)+\eta_{n} \Psi\right)-W\left(\nabla_{\alpha} u_{n} \mid \frac{1}{\varepsilon_{n}} \nabla_{3} u_{n}\right)\right] d x \\
& \leq \liminf _{n \rightarrow+\infty} \int_{\Omega} \partial W\left(\left(\nabla_{\alpha} u_{n} \mid \frac{1}{\varepsilon_{n}} \nabla_{3} u_{n}\right)+\tau_{n} \Psi\right) \cdot \Psi d x
\end{aligned}
$$

for some $\tau_{n} \in\left[0, \eta_{n}\right]$. Lebesgue's Dominated Convergence Theorem in the left hand side, together with Lemma 4.9 of [13] in the right hand side yield

$$
\begin{aligned}
\int_{\Omega} \partial\left(\mathcal{Q} W_{0}\right)\left(\nabla_{\alpha} u\right) \cdot \bar{\Psi} d x & =\lim _{n \rightarrow+\infty} \int_{\Omega} \frac{\mathcal{Q} W_{0}\left(\nabla_{\alpha} u+\eta_{n} \bar{\Psi}\right)-\mathcal{Q} W_{0}\left(\nabla_{\alpha} u\right)}{\eta_{n}} d x \\
& \leq \liminf _{n \rightarrow+\infty} \int_{\Omega} \partial W\left(\left(\nabla_{\alpha} u_{n} \mid \frac{1}{\varepsilon_{n}} \nabla_{3} u_{n}\right)+\tau_{n} \Psi\right) \cdot \Psi d x \\
& =\liminf _{n \rightarrow+\infty} \int_{\Omega} \partial W\left(\nabla_{\alpha} u_{n} \mid \frac{1}{\varepsilon_{n}} \nabla_{3} u_{n}\right) \cdot \Psi d x
\end{aligned}
$$

\section{Convergence of the quasistatic evolution}

The first step of the analysis consists in defining a limit deformation field and a crack. This is done in Lemma 5.1 by means of energy estimates which are possible, thanks to the $L^{\infty}$-boundness assumption (2.8) and to the bound of the prescribed boundary deformation (2.4). The limit deformation $u(t)$ turns out to be the weak $S B V^{p}\left(\Omega^{\prime} ; \mathbb{R}^{3}\right)$-limit of $u^{\varepsilon}(t)$ while the limit crack $\gamma(t)$ is obtained through the convergence of $\Gamma^{\varepsilon}(t)$ in the sense of Definition 4.1. Then, we derive a minimality property for $u(t)$. At time $t=0$ in Lemma 5.2 , we use a $\Gamma$-convergence argument. This is possible because, in the absence of preexisting cracks, the surface term of the energy at time 0 is precisely that introduced in the $\Gamma$-limit analysis of Section 3. Nevertheless, we cannot proceed in this way for the next times in Lemma 5.5 because of the presence of $\Gamma^{\varepsilon}(t)$ in the surface term. We need here to construct directly a sequence thanks to the Jump Transfer Theorem, Theorem 4.4. Then, we show that $(u(t), \gamma(t))$ is a quasistatic evolution for the relaxed model by proving that the energy conservation holds. To do this, we use, on the one hand, the approximation of the Lebesgue integral by Riemann sums in Lemma 5.7, and, on the other hand, the convergence of the total energy at the initial time (that can be proved directly) together with the weak convergence of the stresses and the strong convergence assumption (2.7) in Lemma 5.8. Finally, in Lemma 5.9, we show the convergence of the total energy at any time.

\subsection{Energy estimates and compactness}

Lemma 5.1. There exists a subsequence $\left\{\varepsilon_{n}\right\} \searrow 0^{+}$, a deformation field $u(t) \in S B V^{p}\left(\omega^{\prime} ; \mathbb{R}^{3}\right)$ satisfying $u(t)=g(t) \mathcal{L}^{2}$-a.e. on $\omega^{\prime} \backslash \bar{\omega}$, and a time-increasing crack $\gamma(t) \subset \bar{\omega}$ such that, for every $t \in[0, T]$, $S(u(t)) \widetilde{\subset} \gamma(t)$. Moreover, $\Gamma^{\varepsilon_{n}}(t)$ converges to $\gamma(t)$ in the sense of Definition 4.1, $u^{\varepsilon_{n}}(0) \rightarrow u(0)$ in $S B V^{p}\left(\Omega^{\prime} ; \mathbb{R}^{3}\right)$ and, for every $t \in(0, T]$, there exists a $t$-dependent subsequence $\left\{\varepsilon_{n_{t}}\right\} \subset\left\{\varepsilon_{n}\right\}$ such that $u^{\varepsilon_{n t}}(t) \rightarrow u(t)$ in $S B V^{p}\left(\Omega^{\prime} ; \mathbb{R}^{3}\right)$.

Proof. Firstly, at time $t=0$, we test the minimality of $u^{\varepsilon}(0)$ with $v=g^{\varepsilon}(0)$. Since $S\left(u^{\varepsilon}(0)\right) \cong \Gamma^{\varepsilon}(0)$, we deduce by $(2.4)$ and the growth condition $(2.1)$ that $\mathcal{E}_{\varepsilon}(0) \leq C$. 


\section{JEAN-FranÇOIS BABADJIAN}

Then, we take $v=g^{\varepsilon}(t)$ as test function in (2.5) at time $t$. As $S\left(u^{\varepsilon}(t)\right) \widetilde{\subset} \Gamma^{\varepsilon}(t)$, it follows from (2.4) together with the growth condition (2.1) that

$$
\int_{\Omega} W\left(\nabla_{\alpha} u^{\varepsilon}(t) \mid \frac{1}{\varepsilon} \nabla_{3} u^{\varepsilon}(t)\right) d x \leq \int_{\Omega} W\left(\nabla_{\alpha} g^{\varepsilon}(t) \mid \frac{1}{\varepsilon} \nabla_{3} g^{\varepsilon}(t)\right) d x \leq C .
$$

Thus, Hölder's inequality, (2.2), (2.6) and (5.1) imply the existence of a constant $C>0$, independent of $t$ and $\varepsilon$ such that for every $t \in[0, T], \mathcal{E}_{\varepsilon}(t) \leq C$. Hence, by the coercivity condition $(2.1)$,

$$
\int_{\Omega}\left|\left(\nabla_{\alpha} u^{\varepsilon}(t) \mid \frac{1}{\varepsilon} \nabla_{3} u^{\varepsilon}(t)\right)\right|^{p} d x+\int_{\Gamma^{\varepsilon}(t)}\left|\left(\left(\nu_{\Gamma^{\varepsilon}(t)}\right)_{\alpha} \mid \frac{1}{\varepsilon}\left(\nu_{\Gamma^{\varepsilon}(t)}\right)_{3}\right)\right| d \mathcal{H}^{2} \leq C .
$$

In view of Proposition 4.3, we may find a subsequence $\left\{\varepsilon_{n}\right\} \searrow 0^{+}$and an $\mathcal{H}^{1}$-rectifiable set $\gamma(t) \subset \omega^{\prime}$, increasing in time, such that $\Gamma^{\varepsilon_{n}}(t)$ converges to $\gamma(t)$ in the sense of Definition 4.1. According to Remark $4.2-1$, since $\bar{\omega}$ is compact, $\gamma(t) \widetilde{\subset} \bar{\omega}$ and

$$
2 \mathcal{H}^{1}(\gamma(t)) \leq \liminf _{n \rightarrow+\infty} \mathcal{H}^{2}\left(\Gamma^{\varepsilon_{n}}(t)\right) \leq \liminf _{n \rightarrow+\infty} \int_{\Gamma^{\varepsilon_{n}}(t)}\left|\left(\left(\nu_{\Gamma^{\varepsilon_{n}}(t)}\right)_{\alpha} \mid \frac{1}{\varepsilon_{n}}\left(\nu_{\Gamma^{\varepsilon_{n}}(t)}\right)_{3}\right)\right| d \mathcal{H}^{2}
$$

As $S\left(u^{\varepsilon}(t)\right) \widetilde{\subset} \Gamma^{\varepsilon}(t)$ and $u^{\varepsilon}(t)=g^{\varepsilon}(t) \mathcal{L}^{3}$-a.e. on $\left[\omega^{\prime} \backslash \bar{\omega}\right] \times I$, we have by $(2.8)$ and $(5.2)$

$$
\begin{aligned}
\left\|u^{\varepsilon}(t)\right\|_{L^{\infty}\left(\Omega^{\prime} ; \mathbb{R}^{3}\right)}+\int_{\Omega^{\prime}} \mid\left(\nabla_{\alpha} u^{\varepsilon}(t)\right. & \left.\mid \frac{1}{\varepsilon} \nabla_{3} u^{\varepsilon}(t)\right)\left.\right|^{p} d x \\
& +\int_{S\left(u^{\varepsilon}(t)\right)}\left|\left(\left(\nu_{u^{\varepsilon}(t)}\right)_{\alpha} \mid \frac{1}{\varepsilon}\left(\nu_{u^{\varepsilon}(t)}\right)_{3}\right)\right| d \mathcal{H}^{2} \leq C,
\end{aligned}
$$

for some constant $C>0$ independent of $\varepsilon>0$ and $t \in[0, T]$. We insist, once again, on the fact that we do not try to justify the boundness assumption on $u^{\varepsilon}(t)$. In view of Lemma 2.2, there exists a further subsequence of $\left\{\varepsilon_{n}\right\}$ (still denoted by $\left.\left\{\varepsilon_{n}\right\}\right)$ and $u(0) \in S B V^{p}\left(\omega^{\prime} ; \mathbb{R}^{3}\right)$ such that $u^{\varepsilon_{n}}(0) \rightarrow u(0)$ in $S B V^{p}\left(\Omega^{\prime} ; \mathbb{R}^{3}\right)$. Moreover, as $u^{\varepsilon_{n}}(0)=g^{\varepsilon_{n}}(0) \mathcal{L}^{3}$-a.e. on $\left[\omega^{\prime} \backslash \bar{\omega}\right] \times I$, from $(2.7)$ we get $u(0)=g(0) \mathcal{L}^{2}$-a.e. on $\omega^{\prime} \backslash \bar{\omega}$. Thanks to condition (a) of Definition 4.1, we deduce that $S(u(0)) \widetilde{\subset} \gamma(0)$.

We set for a.e. $t \in[0, T]$,

$$
\left\{\begin{aligned}
\theta_{n}(t) & :=\int_{\Omega} \partial W\left(\nabla_{\alpha} u^{\varepsilon_{n}}(t) \mid \frac{1}{\varepsilon_{n}} \nabla_{3} u^{\varepsilon_{n}}(t)\right) \cdot\left(\nabla_{\alpha} \dot{g}^{\varepsilon_{n}}(t) \mid \frac{1}{\varepsilon_{n}} \nabla_{3} \dot{g}^{\varepsilon_{n}}(t)\right) d x, \\
\theta(t) & :=\limsup _{n \rightarrow+\infty} \theta_{n}(t) .
\end{aligned}\right.
$$

From (2.2), (2.4), (5.2), $\theta \in L^{1}(0, T)$ and by virtue of Fatou's Lemma

$$
\limsup _{n \rightarrow+\infty} \int_{0}^{t} \theta_{n}(s) d s \leq \int_{0}^{t} \theta(s) d s .
$$

For a.e. $t \in[0, T]$, we extract a $t$-dependent subsequence $\left\{n_{t}\right\}$ such that

$$
\theta(t)=\lim _{n_{t} \rightarrow+\infty} \theta_{n_{t}}(t) .
$$

Lemma 2.2 implies that for every $t \in(0, T]$, upon extracting a further subsequence (not relabeled), $u^{\varepsilon_{n_{t}}}(t) \rightarrow u(t)$ in $S B V^{p}\left(\Omega^{\prime} ; \mathbb{R}^{3}\right)$ for some $u(t) \in S B V^{p}\left(\omega^{\prime} ; \mathbb{R}^{3}\right)$. Moreover, as $u^{\varepsilon_{n_{t}}}(t)=g^{\varepsilon_{n_{t}}}(t) \mathcal{L}^{3}$-a.e. on $\left[\omega^{\prime} \backslash \bar{\omega}\right] \times I$, from $(2.7)$, we get $u(t)=g(t) \mathcal{L}^{2}$-a.e. on $\omega^{\prime} \backslash \bar{\omega}$. By condition (a) of Definition 4.1 , we get that $S(u(t)) \widetilde{\subset} \gamma(t)$. 


\subsection{Minimality property}

For all $t \in[0, T]$, we define the limit energy by

$$
\mathcal{E}(t):=2 \int_{\omega} \mathcal{Q} W_{0}\left(\nabla_{\alpha} u(t)\right) d x_{\alpha}+2 \mathcal{H}^{1}(\gamma(t)) .
$$

Our goal is to show that $u(t)$ satisfies some minimality property inherited from that of $u^{\varepsilon}(t)$. We will distinguish the initial time from the subsequent times. At time $t=0$, we will further show the convergence of the bulk and the surface energy to their two-dimensional counterpart respectively, for the subsequence $\left\{\varepsilon_{n}\right\}$. Concerning the next times, we will only be able to prove the convergence of the bulk energy toward its two-dimensional analogue, for the $t$-dependent subsequence $\left\{\varepsilon_{n_{t}}\right\}$. The convergence of the total energy, or equivalently of the surface energy, will be established later in Lemma 5.9 for a subsequence independent of the time.

Lemma 5.2. At time $t=0, u(0)$ minimizes

$$
v \mapsto 2 \int_{\omega} \mathcal{Q} W_{0}\left(\nabla_{\alpha} v\right) d x_{\alpha}+2 \mathcal{H}^{1}(S(v)),
$$

among $\left\{v \in S B V^{p}\left(\omega^{\prime} ; \mathbb{R}^{3}\right): \quad v=g(0)\right.$ a.e. on $\left.\omega^{\prime} \backslash \bar{\omega}\right\}$. Moreover, $\gamma(0) \cong S(u(0))$ and we have

$$
\left\{\begin{array}{l}
\int_{\Omega} W\left(\nabla_{\alpha} u^{\varepsilon_{n}}(0) \mid \frac{1}{\varepsilon_{n}} \nabla_{3} u^{\varepsilon_{n}}(0)\right) d x \rightarrow 2 \int_{\omega} \mathcal{Q} W_{0}\left(\nabla_{\alpha} u(0)\right) d x_{\alpha}, \\
\int_{\Gamma^{\varepsilon_{n}}(0)}\left|\left(\left(\nu_{\Gamma^{\varepsilon_{n}}(0)}\right)_{\alpha} \mid \frac{1}{\varepsilon_{n}}\left(\nu_{\Gamma^{\varepsilon_{n}}(0)}\right)_{3}\right)\right| d \mathcal{H}^{2} \rightarrow 2 \mathcal{H}^{1}(\gamma(0)) .
\end{array}\right.
$$

In particular, $\mathcal{E}_{\varepsilon_{n}}(0) \rightarrow \mathcal{E}(0)$ and

$$
\partial W\left(\nabla_{\alpha} u^{\varepsilon_{n}}(0) \mid \frac{1}{\varepsilon_{n}} \nabla_{3} u^{\varepsilon_{n}}(0)\right) \rightarrow\left(\partial\left(\mathcal{Q} W_{0}\right)\left(\nabla_{\alpha} u(0)\right) \mid 0\right) \quad \text { in } L^{p^{\prime}}\left(\Omega ; \mathbb{R}^{3 \times 3}\right) .
$$

Proof. Let $v \in S B V^{p}\left(\omega^{\prime} ; \mathbb{R}^{3}\right)$ such that $v=g(0) \mathcal{L}^{2}$-a.e. on $\omega^{\prime} \backslash \bar{\omega}$. By virtue of Corollary 3.11, there exists a sequence $\left\{w_{n}\right\} \subset S B V^{p}\left(\Omega^{\prime} ; \mathbb{R}^{3}\right)$ satisfying $w_{n}=g^{\varepsilon_{n}}(0) \mathcal{L}^{3}$-a.e. on $\left[\omega^{\prime} \backslash \bar{\omega}\right] \times I, w_{n} \rightarrow v$ in $L^{1}\left(\Omega^{\prime} ; \mathbb{R}^{3}\right)$ and

$$
\begin{aligned}
& 2 \int_{\omega} \mathcal{Q} W_{0}\left(\nabla_{\alpha} v\right) d x_{\alpha}+2 \mathcal{H}^{1}(S(v)) \\
&=\lim _{n \rightarrow+\infty}\left[\int_{\Omega} W\left(\nabla_{\alpha} w_{n} \mid \frac{1}{\varepsilon_{n}} \nabla_{3} w_{n}\right) d x+\int_{S\left(w_{n}\right)}\left|\left(\left(\nu_{w_{n}}\right)_{\alpha} \mid \frac{1}{\varepsilon_{n}}\left(\nu_{w_{n}}\right)_{3}\right)\right| d \mathcal{H}^{2}\right] .
\end{aligned}
$$

Taking $w_{n}$ as test function in the minimality condition for $u^{\varepsilon_{n}}(0)$ we get

$$
\begin{gathered}
\int_{\Omega} W\left(\nabla_{\alpha} u^{\varepsilon_{n}}(0) \mid \frac{1}{\varepsilon_{n}} \nabla_{3} u^{\varepsilon_{n}}(0)\right) d x+\int_{S\left(u^{\varepsilon_{n}}(0)\right)}\left|\left(\left(\nu_{u^{\varepsilon_{n}}(0)}\right)_{\alpha} \mid \frac{1}{\varepsilon_{n}}\left(\nu_{u^{\varepsilon_{n}}(0)}\right)_{3}\right)\right| d \mathcal{H}^{2} \\
\leq \int_{\Omega} W\left(\nabla_{\alpha} w_{n} \mid \frac{1}{\varepsilon_{n}} \nabla_{3} w_{n}\right) d x+\int_{S\left(w_{n}\right)}\left|\left(\left(\nu_{w_{n}}\right)_{\alpha} \mid \frac{1}{\varepsilon_{n}}\left(\nu_{w_{n}}\right)_{3}\right)\right| d \mathcal{H}^{2}
\end{gathered}
$$

Remark 3.2 and Theorem 5.29 in [2] yield

$$
\begin{aligned}
2 \int_{\omega} \mathcal{Q} W_{0}\left(\nabla_{\alpha} u(0)\right) d x_{\alpha} & \leq \liminf _{n \rightarrow+\infty} \int_{\Omega} \mathcal{Q} W_{0}\left(\nabla_{\alpha} u^{\varepsilon_{n}}(0)\right) d x \\
& \leq \liminf _{n \rightarrow+\infty} \int_{\Omega} W\left(\nabla_{\alpha} u^{\varepsilon_{n}}(0) \mid \frac{1}{\varepsilon_{n}} \nabla_{3} u^{\varepsilon_{n}}(0)\right) d x
\end{aligned}
$$


and thanks to (5.3) together with the fact that $\Gamma^{\varepsilon_{n}}(0) \cong S\left(u^{\varepsilon_{n}}(0)\right)$,

$$
\begin{aligned}
2 \mathcal{H}^{1}(\gamma(0)) & \leq \liminf _{n \rightarrow+\infty} \mathcal{H}^{2}\left(\Gamma^{\varepsilon_{n}}(0)\right) \\
& =\liminf _{n \rightarrow+\infty} \mathcal{H}^{2}\left(S\left(u^{\varepsilon_{n}}(0)\right)\right) \\
& \leq \liminf _{n \rightarrow+\infty} \int_{S\left(u^{\varepsilon_{n}}(0)\right)}\left|\left(\left(\nu_{u^{\varepsilon_{n}}(0)}\right)_{\alpha} \mid \frac{1}{\varepsilon_{n}}\left(\nu_{u^{\varepsilon_{n}}(0)}\right)_{3}\right)\right| d \mathcal{H}^{2} .
\end{aligned}
$$

Finally, from (5.8), (5.9), (5.10) and (5.11) we get by letting $n \rightarrow+\infty$,

$$
2 \int_{\omega} \mathcal{Q} W_{0}\left(\nabla_{\alpha} u(0)\right) d x_{\alpha}+2 \mathcal{H}^{1}(\gamma(0)) \leq 2 \int_{\omega} \mathcal{Q} W_{0}\left(\nabla_{\alpha} v\right) d x_{\alpha}+2 \mathcal{H}^{1}(S(v)) .
$$

Taking $v=u(0)$ in the previous inequality, we observe that $\mathcal{H}^{1}(\gamma(0)) \leq \mathcal{H}^{1}(S(u(0)))$, which implies, as $S(u(0)) \widetilde{\subset} \gamma(0)$, that $S(u(0)) \cong \gamma(0)$. It establishes the minimality property satisfied by $u(0)$. Taking still $v=u(0),(5.9)$ and (5.11) give

$$
\limsup _{n \rightarrow+\infty} \int_{\Omega} W\left(\nabla_{\alpha} u^{\varepsilon_{n}}(0) \mid \frac{1}{\varepsilon_{n}} \nabla_{3} u^{\varepsilon_{n}}(0)\right) d x \leq 2 \int_{\omega} \mathcal{Q} W_{0}\left(\nabla_{\alpha} u(0)\right) d x_{\alpha} .
$$

and this shows with (5.10) that

$$
\int_{\Omega} W\left(\nabla_{\alpha} u^{\varepsilon_{n}}(0) \mid \frac{1}{\varepsilon_{n}} \nabla_{3} u^{\varepsilon_{n}}(0)\right) d x \rightarrow 2 \int_{\omega} \mathcal{Q} W_{0}\left(\nabla_{\alpha} u(0)\right) d x_{\alpha} .
$$

We report in (5.9) and obtain

$$
\limsup _{n \rightarrow+\infty} \int_{S\left(u^{\varepsilon_{n}}(0)\right)}\left|\left(\left(\nu_{u^{\varepsilon_{n}}(0)}\right)_{\alpha} \mid \frac{1}{\varepsilon_{n}}\left(\nu_{u^{\varepsilon_{n}}(0)}\right)_{3}\right)\right| d \mathcal{H}^{2} \leq 2 \mathcal{H}^{1}(S(u(0))),
$$

which implies together with (5.11) that

$$
\int_{S\left(u^{\varepsilon_{n}}(0)\right)}\left|\left(\left(\nu_{u^{\varepsilon_{n}}(0)}\right)_{\alpha} \mid \frac{1}{\varepsilon_{n}}\left(\nu_{u^{\varepsilon_{n}}(0)}\right)_{3}\right)\right| d \mathcal{H}^{2} \rightarrow 2 \mathcal{H}^{1}(S(u(0))) .
$$

This yields that $\mathcal{E}_{\varepsilon_{n}}(0) \rightarrow \mathcal{E}(0)$ and the convergence of the stresses follows from Lemma 4.8.

Remark 5.3. It is immediate from the previous lemma that $u(0)$ minimizes

$$
v \mapsto 2 \int_{\omega} \mathcal{Q} W_{0}\left(\nabla_{\alpha} v\right) d x_{\alpha}+2 \mathcal{H}^{1}(S(v) \backslash \gamma(0)),
$$

among $\left\{v \in S B V^{p}\left(\omega^{\prime} ; \mathbb{R}^{3}\right): \quad v=g(0)\right.$ a.e. on $\left.\omega^{\prime} \backslash \bar{\omega}\right\}$.

Remark 5.4. Note that the previous result holds because we did not allow the body to contain a preexisting crack. Indeed, in this case, since the energy we are minimizing at the initial time is the same as the functional involved in the $\Gamma$-limit analysis, we can take as competitor in the minimization a recovery sequence. It permits us to show the convergence of the total energy at time $t=0$; that is essential if one is to prove that it still holds true at subsequent times in Lemma 5.8. If we had considered a body containing a preexisting crack, we would be unable to obtain such a convergence, but only a convergence of the bulk energy. Indeed, if $\Gamma_{0}^{\varepsilon} \subset \bar{\omega} \times I$ denoted a preexisting (rescaled) crack with bounded scaled surface energy, then, according to the formulation in [13], $\left(u_{0}^{\varepsilon}, \Gamma_{0}^{\varepsilon}\right)$ would have to minimize

$$
(v, \Gamma) \mapsto \int_{\Omega} W\left(\nabla_{\alpha} v \mid \frac{1}{\varepsilon} \nabla_{3} v\right) d x+\int_{\Gamma}\left|\left(\left(\nu_{\Gamma}\right)_{\alpha} \mid \frac{1}{\varepsilon}\left(\nu_{\Gamma}\right)_{3}\right)\right| d \mathcal{H}^{2}
$$




\section{QUASISTATIC EVOLUTION OF A BRITTLE THIN FILM}

among every $\mathcal{H}^{2}$-rectifiable crack $\Gamma \subset \bar{\omega} \times I$ with $\Gamma_{0}^{\varepsilon} \widetilde{\subset} \Gamma$, and every deformation $v \in S B V^{p}\left(\Omega^{\prime} ; \mathbb{R}^{3}\right)$ such that $v=g^{\varepsilon}(t)$ a.e. on $\left[\omega^{\prime} \backslash \bar{\omega}\right] \times I$ and $S(v) \widetilde{\subset} \Gamma$. In particular, setting $\Gamma:=\Gamma_{0}^{\varepsilon} \cup S(v)$ for all $v \in S B V^{p}\left(\Omega^{\prime} ; \mathbb{R}^{3}\right)$ satisfying $v=g^{\varepsilon}(0)$ a.e. on $\left[\omega^{\prime} \backslash \bar{\omega}\right] \times I$, we would get that $u_{0}^{\varepsilon}$ must minimize

$$
v \mapsto \int_{\Omega} W\left(\nabla_{\alpha} v \mid \frac{1}{\varepsilon} \nabla_{3} v\right) d x+\int_{S(v) \backslash \Gamma_{0}^{\varepsilon}}\left|\left(\left(\nu_{v}\right)_{\alpha} \mid \frac{1}{\varepsilon}\left(\nu_{v}\right)_{3}\right)\right| d \mathcal{H}^{2}
$$

among such $v$ 's. Hence, since by Theorem 3.15 in [13] (or Theorem 2.1 in [14]) $\left(u^{\varepsilon}(0), \Gamma^{\varepsilon}(0)\right)=\left(u_{0}^{\varepsilon}, \Gamma_{0}^{\varepsilon}\right)$, the argument used in the proof of Lemma 5.2 would not hold anymore. We would only be able to state, as in the following Lemma 5.5, the convergence of the bulk energy. Unfortunately, the convergence of the surface energy would then remain an open question.

We are now going to state a minimality property satisfied by $u(t)$ for $t \in(0, T]$. The following result ensures the convergence of the three-dimensional bulk energy to its two-dimensional counterpart for a $t$-dependent subsequence. But the convergence of the total energy, or equivalently of the surface energy, cannot be established at this stage in a manner similar to that used in Lemma 5.2 at the initial time.

Lemma 5.5. For every $t \in(0, T], u(t)$ minimizes

$$
v \mapsto 2 \int_{\omega} \mathcal{Q} W_{0}\left(\nabla_{\alpha} v\right) d x_{\alpha}+2 \mathcal{H}^{1}(S(v) \backslash \gamma(t)),
$$

among $\left\{v \in S B V^{p}\left(\omega^{\prime} ; \mathbb{R}^{3}\right): \quad v=g(t)\right.$ a.e. on $\left.\omega^{\prime} \backslash \bar{\omega}\right\}$. Moreover, we have

$$
\int_{\Omega} W\left(\nabla_{\alpha} u^{\varepsilon_{n_{t}}}(t) \mid \frac{1}{\varepsilon_{n_{t}}} \nabla_{3} u^{\varepsilon_{n_{t}}}(t)\right) d x \rightarrow 2 \int_{\omega} \mathcal{Q} W_{0}\left(\nabla_{\alpha} u(t)\right) d x_{\alpha} .
$$

In particular,

$$
\partial W\left(\nabla_{\alpha} u^{\varepsilon_{n_{t}}}(t) \mid \frac{1}{\varepsilon_{n_{t}}} \nabla_{3} u^{\varepsilon_{n_{t}}}(t)\right) \rightarrow\left(\partial\left(\mathcal{Q} W_{0}\right)\left(\nabla_{\alpha} u(t) \mid 0\right)\right) \quad \text { in } L^{p^{\prime}}\left(\Omega ; \mathbb{R}^{3 \times 3}\right)
$$

and thus, for a.e. $t \in[0, T]$,

$$
\theta(t)=2 \int_{\omega} \partial\left(\mathcal{Q} W_{0}\right)\left(\nabla_{\alpha} u(t)\right) \cdot \nabla_{\alpha} \dot{g}(t) d x_{\alpha}
$$

Proof. We first prove the minimality property. Unlike Lemma 5.2, we cannot use a $\Gamma$-convergence argument because of the presence of an $\varepsilon$-dependent crack in the surface term. We will construct a minimizing sequence with the help of the Jump Transfer Theorem.

Let $w \in S B V^{p}\left(\omega^{\prime} ; \mathbb{R}^{3}\right)$ such that $w=g(t) \mathcal{L}^{2}$-a.e. on $\omega^{\prime} \backslash \bar{\omega}$. Since $\Gamma^{\varepsilon_{n}}(t)$ converges to $\gamma(t)$ in the sense of Definition 4.1, from Theorem 4.6, there exists a sequence $\left\{w_{n}\right\} \subset S B V^{p}\left(\Omega^{\prime} ; \mathbb{R}^{3}\right)$ satisfying $w_{n}=w=g(t)$ a.e. on $\left[\omega^{\prime} \backslash \bar{\omega}\right] \times I, w_{n} \rightarrow w$ in $L^{1}\left(\Omega^{\prime} ; \mathbb{R}^{3}\right)$ and

$$
\left\{\begin{array}{l}
\left(\nabla_{\alpha} w_{n} \mid \frac{1}{\varepsilon_{n}} \nabla_{3} w_{n}\right) \rightarrow\left(\nabla_{\alpha} w \mid 0\right) \text { in } L^{p}\left(\Omega^{\prime} ; \mathbb{R}^{3 \times 3}\right), \\
\limsup _{n \rightarrow+\infty} \int_{S\left(w_{n}\right) \backslash \Gamma^{\varepsilon_{n}}(t)}\left|\left(\left(\nu_{w_{n}}\right)_{\alpha} \mid \frac{1}{\varepsilon_{n}}\left(\nu_{w_{n}}\right)_{3}\right)\right| d \mathcal{H}^{2} \leq 2 \mathcal{H}^{1}(S(w) \backslash \gamma(t)) .
\end{array}\right.
$$

A measurable selection criterion (see e.g. [15]) together with the coercivity condition (2.1) imply the existence of $z \in L^{p}\left(\omega ; \mathbb{R}^{3}\right)$ such that $W_{0}\left(\nabla_{\alpha} w\right)=W\left(\nabla_{\alpha} w \mid z\right) \mathcal{L}^{2}$-a.e. in $\omega$. By density, there exists a 


\section{JEAN-FranÇOIS BABADJIAN}

sequence $z_{j} \in \mathcal{C}_{c}^{\infty}\left(\Omega ; \mathbb{R}^{3}\right)$ such that $z_{j} \rightarrow H(t)-z$ in $L^{p}\left(\Omega ; \mathbb{R}^{3}\right)$ where $H(t)$ is defined in (2.7). Denoting by $b_{j}:=\int_{-1}^{x_{3}} z_{j}(\cdot, s) d s$, we take $w_{n}+g^{\varepsilon_{n}}(t)-g(t)-\varepsilon_{n} b_{j}$ as test function in (2.5) and we get

$$
\begin{aligned}
& \int_{\Omega} W\left(\nabla_{\alpha} u^{\varepsilon_{n}}(t) \mid \frac{1}{\varepsilon_{n}} \nabla_{3} u^{\varepsilon_{n}}(t)\right) d x \\
& \leq \int_{\Omega} W\left(\nabla_{\alpha} w_{n}+\nabla_{\alpha} g^{\varepsilon_{n}}(t)-\nabla_{\alpha} g(t)-\varepsilon_{n} \nabla_{\alpha} b_{j} \mid \frac{1}{\varepsilon_{n}} \nabla_{3} w_{n}+\frac{1}{\varepsilon_{n}} \nabla_{3} g^{\varepsilon_{n}}(t)-z_{j}\right) d x \\
& \quad+\int_{S\left(w_{n}\right) \backslash \Gamma^{\varepsilon_{n}}(t)}\left|\left(\left(\nu_{w_{n}}\right)_{\alpha} \mid \frac{1}{\varepsilon_{n}}\left(\nu_{w_{n}}\right)_{3}\right)\right| d \mathcal{H}^{2} .
\end{aligned}
$$

We replace $n$ by $n_{t}$ (see Lemma 5.1) and pass to the limit when $n_{t}$ tends to $+\infty$. In view of Theorem 5.29 in [2] and of Remark 3.2,

$$
\begin{aligned}
2 \int_{\omega} \mathcal{Q} W_{0}\left(\nabla_{\alpha} u(t)\right) d x_{\alpha} & \leq \liminf _{n_{t} \rightarrow+\infty} \int_{\Omega} \mathcal{Q} W_{0}\left(\nabla_{\alpha} u^{\varepsilon_{n_{t}}}(t)\right) d x \\
& \leq \liminf _{n_{t} \rightarrow+\infty} \int_{\Omega} W\left(\nabla_{\alpha} u^{\varepsilon_{n_{t}}}(t) \mid \frac{1}{\varepsilon_{n_{t}}} \nabla_{3} u^{\varepsilon_{n_{t}}}(t)\right) d x .
\end{aligned}
$$

Thus, using (2.7) and (5.13) in the right hand side, we get

$$
2 \int_{\omega} \mathcal{Q} W_{0}\left(\nabla_{\alpha} u(t)\right) d x_{\alpha} \leq \int_{\Omega} W\left(\nabla_{\alpha} w \mid H(t)-z_{j}\right) d x+2 \mathcal{H}^{1}(S(w) \backslash \gamma(t)) .
$$

Passing to the limit when $j \rightarrow+\infty$ we obtain

$$
\begin{aligned}
2 \int_{\omega} \mathcal{Q} W_{0}\left(\nabla_{\alpha} u(t)\right) d x_{\alpha} & \leq \int_{\Omega} W\left(\nabla_{\alpha} w \mid z\right) d x+2 \mathcal{H}^{1}(S(w) \backslash \gamma(t)) \\
& =2 \int_{\omega} W_{0}\left(\nabla_{\alpha} w\right) d x_{\alpha}+2 \mathcal{H}^{1}(S(w) \backslash \gamma(t)) .
\end{aligned}
$$

We would like to replace $W_{0}$ by its quasiconvexification in the previous relation. To this end, we use a relaxation argument. First of all, we approach $\gamma(t)$ from inside by a compact set, so as to work on an open subset of $\omega$. This is possible because, since $\mathcal{H}^{1}(\gamma(t))<+\infty$, then $\mathcal{H}_{\lfloor\gamma(t)}^{1}$ is a Radon measure. Thus, for any $\eta>0$, there exists a compact set $K_{t}^{\eta} \subset \gamma(t)$ such that $\mathcal{H}^{1}\left(\gamma(t) \backslash K_{t}^{\eta}\right) \leq \eta$. In particular,

$$
2 \int_{\omega} \mathcal{Q} W_{0}\left(\nabla_{\alpha} u(t)\right) d x_{\alpha} \leq 2 \int_{\omega} W_{0}\left(\nabla_{\alpha} w\right) d x_{\alpha}+2 \mathcal{H}^{1}\left(S(w) \backslash K_{t}^{\eta}\right) .
$$

Let $v \in S B V^{p}\left(\omega^{\prime} ; \mathbb{R}^{3}\right)$ satisfying $v=g(t) \mathcal{L}^{2}$-a.e. on $\omega^{\prime} \backslash \bar{\omega}$. In view of Theorem 8.1 together with Remark 8.2 in [7] and arguing as in the proof of Lemma 3.10 and Corollary 3.11, it is easily deduced that there exists a sequence $\left\{w_{k}\right\} \subset S B V^{p}\left(\omega^{\prime} ; \mathbb{R}^{3}\right)$ such that $w_{k} \rightarrow v$ in $L^{1}\left(\omega^{\prime} ; \mathbb{R}^{3}\right), w_{k}=g(t) \mathcal{L}^{2}$-a.e. on $\omega^{\prime} \backslash \bar{\omega}$ and

$$
\int_{\omega} \mathcal{Q} W_{0}\left(\nabla_{\alpha} v\right) d x_{\alpha}+\mathcal{H}^{1}\left(S(v) \backslash K_{t}^{\eta}\right)=\lim _{k \rightarrow+\infty}\left[\int_{\omega} W_{0}\left(\nabla_{\alpha} w_{k}\right) d x_{\alpha}+\mathcal{H}^{1}\left(S\left(w_{k}\right) \backslash K_{t}^{\eta}\right)\right] .
$$

In (5.15), we replace $w$ by $w_{k}$ and we pass to the limit when $k \rightarrow+\infty$; we get

$$
\begin{aligned}
2 \int_{\omega} \mathcal{Q} W_{0}\left(\nabla_{\alpha} u(t)\right) d x_{\alpha} & \leq 2 \int_{\omega} \mathcal{Q} W_{0}\left(\nabla_{\alpha} v\right) d x_{\alpha}+2 \mathcal{H}^{1}\left(S(v) \backslash K_{t}^{\eta}\right) \\
& \leq 2 \int_{\omega} \mathcal{Q} W_{0}\left(\nabla_{\alpha} v\right) d x_{\alpha}+2 \mathcal{H}^{1}(S(v) \backslash \gamma(t))+2 \eta
\end{aligned}
$$


The minimality property follows after letting $\eta \rightarrow 0$.

Concerning the convergence of the bulk energy, the previous calculation with $v=u(t)$ and the fact that $S(u(t)) \widetilde{\subset} \gamma(t)$ yield

$$
\limsup _{n \rightarrow+\infty} \int_{\Omega} W\left(\nabla_{\alpha} u^{\varepsilon_{n}}(t) \mid \frac{1}{\varepsilon_{n}} \nabla_{3} u^{\varepsilon_{n}}(t)\right) d x \leq 2 \int_{\omega} \mathcal{Q} W_{0}\left(\nabla_{\alpha} u(t)\right) d x_{\alpha} .
$$

Note that (5.16) holds for the sequence $\left\{\varepsilon_{n}\right\}$ which is independent of the time. Thus, from (5.14), we deduce that

$$
\int_{\Omega} W\left(\nabla_{\alpha} u^{\varepsilon_{n_{t}}}(t) \mid \frac{1}{\varepsilon_{n_{t}}} \nabla_{3} u^{\varepsilon_{n_{t}}}(t)\right) d x \rightarrow 2 \int_{\omega} \mathcal{Q} W_{0}\left(\nabla_{\alpha} u(t)\right) d x_{\alpha} .
$$

In particular, Lemma 4.8 implies the convergence of the stresses and thanks to (2.7), (5.4) and (5.6), we have for a.e. $t \in[0, T]$,

$$
\begin{aligned}
& \theta(t)=\lim _{n_{t} \rightarrow+\infty} \int_{\Omega} \partial W\left(\nabla_{\alpha} u^{\varepsilon_{n_{t}}}(t) \mid \frac{1}{\varepsilon_{n_{t}}} \nabla_{3} u^{\varepsilon_{n_{t}}}(t)\right) \cdot\left(\nabla_{\alpha} \dot{g}^{\varepsilon_{n_{t}}}(t) \mid \frac{1}{\varepsilon_{n_{t}}} \nabla_{3} \dot{g}^{\varepsilon_{n}}(t)\right) d x \\
& =\int_{\Omega}\left(\partial\left(\mathcal{Q} W_{0}\right)\left(\nabla_{\alpha} u(t)\right) \mid 0\right) \cdot\left(\nabla_{\alpha} \dot{g}(t) \mid \dot{H}(t)\right) d x \\
& =2 \int_{\omega} \partial\left(\mathcal{Q} W_{0}\right)\left(\nabla_{\alpha} u(t)\right) \cdot \nabla_{\alpha} \dot{g}(t) d x_{\alpha} \text {. }
\end{aligned}
$$

Remark 5.6. According to Remark 5.3 and Lemma 5.5, for every $t \in[0, T]$, the function $u(t)$ minimizes

$$
v \mapsto 2 \int_{\omega} \mathcal{Q} W_{0}\left(\nabla_{\alpha} v\right) d x_{\alpha}+2 \mathcal{H}^{1}(S(v) \backslash \gamma(t)),
$$

among $\left\{v \in S B V^{p}\left(\omega^{\prime} ; \mathbb{R}^{3}\right): \quad v=g(t)\right.$ a.e. on $\left.\omega^{\prime} \backslash \bar{\omega}\right\}$. Equivalently, the pair $(u(t), \gamma(t))$ satisfies the following unilateral minimality property:

$$
2 \int_{\omega} \mathcal{Q} W_{0}\left(\nabla_{\alpha} u(t)\right) d x_{\alpha}+2 \mathcal{H}^{1}(\gamma(t)) \leq 2 \int_{\omega} \mathcal{Q} W_{0}\left(\nabla_{\alpha} v\right) d x_{\alpha}+2 \mathcal{H}^{1}\left(\gamma^{\prime}\right),
$$

for every $\mathcal{H}^{1}$-rectifiable set $\gamma^{\prime} \subset \bar{\omega}$ such that $\gamma(t) \widetilde{\subset} \gamma^{\prime}$ and every $v \in S B V^{p}\left(\omega^{\prime} ; \mathbb{R}^{3}\right)$ satisfying $v=g(t)$ a.e. on $\omega^{\prime} \backslash \bar{\omega}$ and $S(v) \widetilde{\subset} \gamma^{\prime}$. Indeed, for such pairs $\left(v, \gamma^{\prime}\right)$, from (5.17) we get that

$$
\begin{aligned}
2 \int_{\omega} \mathcal{Q} W_{0}\left(\nabla_{\alpha} u(t)\right) d x_{\alpha} & \leq 2 \int_{\omega} \mathcal{Q} W_{0}\left(\nabla_{\alpha} v\right) d x_{\alpha}+2 \mathcal{H}^{1}(S(v) \backslash \gamma(t)) \\
& \leq 2 \int_{\omega} \mathcal{Q} W_{0}\left(\nabla_{\alpha} v\right) d x_{\alpha}+2 \mathcal{H}^{1}\left(\gamma^{\prime} \backslash \gamma(t)\right) \\
& =2 \int_{\omega} \mathcal{Q} W_{0}\left(\nabla_{\alpha} v\right) d x_{\alpha}+2 \mathcal{H}^{1}\left(\gamma^{\prime}\right)-2 \mathcal{H}^{1}(\gamma(t))
\end{aligned}
$$

where the second inequality holds since $S(v) \widetilde{\subset} \gamma^{\prime}$ and the last equality because $\gamma(t) \widetilde{\subset} \gamma^{\prime}$. On the other hand, (5.17) follows from (5.18) by taking $\gamma^{\prime}:=S(v) \cup \gamma(t)$.

\subsection{Energy conservation}

The last step in proving that $(u(t), \gamma(t))$ is a quasistatic evolution relative to the boundary data $g(t)$ consists in showing that the two-dimensional total energy $\mathcal{E}(t)$ defined in $(5.7)$ is absolutely continuous in time. This is the aim of Lemmas 5.7 and 5.8 that follow. 


\section{JEAN-FRANÇOIS BABADJIAN}

Lemma 5.7. For every $t \in[0, T]$,

$$
\mathcal{E}(t) \geq \mathcal{E}(0)+2 \int_{0}^{t} \int_{\omega} \partial\left(\mathcal{Q} W_{0}\right)\left(\nabla_{\alpha} u(\tau)\right) \cdot \nabla_{\alpha} \dot{g}(\tau) d x_{\alpha} d \tau
$$

Proof. We proceed as in [14] by approximation of the Lebesgue integral by Riemann sums. Let $s<t$, at time $s$ we test the minimality of $u(s)$ against $u(t)+g(s)-g(t)$. By Lemma 5.5,

$$
2 \int_{\omega} \mathcal{Q} W_{0}\left(\nabla_{\alpha} u(s)\right) d x_{\alpha} \leq 2 \int_{\omega} \mathcal{Q} W_{0}\left(\nabla_{\alpha} u(t)+\nabla_{\alpha} g(s)-\nabla_{\alpha} g(t)\right) d x_{\alpha}+2 \mathcal{H}^{1}(S(u(t)) \backslash \gamma(s)) .
$$

Thus, since $S(u(t)) \widetilde{\subset} \gamma(t)$ and $\gamma(s) \subset \gamma(t)$,

$$
\begin{aligned}
\mathcal{E}(s) & =2 \int_{\omega} \mathcal{Q} W_{0}\left(\nabla_{\alpha} u(s)\right) d x_{\alpha}+2 \mathcal{H}^{1}(\gamma(s)) \\
& \leq 2 \int_{\omega} \mathcal{Q} W_{0}\left(\nabla_{\alpha} u(t)+\nabla_{\alpha} g(s)-\nabla_{\alpha} g(t)\right) d x_{\alpha}+2 \mathcal{H}^{1}(\gamma(t)) \\
& =2 \int_{\omega} \mathcal{Q} W_{0}\left(\nabla_{\alpha} u(t)+\nabla_{\alpha} g(s)-\nabla_{\alpha} g(t)\right) d x_{\alpha}-2 \int_{\omega} \mathcal{Q} W_{0}\left(\nabla_{\alpha} u(t)\right) d x_{\alpha}+\mathcal{E}(t) .
\end{aligned}
$$

It implies that for some $\rho(s, t) \in[0,1]$,

$$
\mathcal{E}(t)-\mathcal{E}(s) \geq 2 \int_{\omega}\left[\partial\left(\mathcal{Q} W_{0}\right)\left(\nabla_{\alpha} u(t)+\rho(s, t) \int_{s}^{t} \nabla_{\alpha} \dot{g}(\tau) d \tau\right) \cdot \int_{s}^{t} \nabla_{\alpha} \dot{g}(\tau) d \tau\right] d x .
$$

Fix $t \in[0, T]$, thanks to Lemma 4.12 in [13], there exists a subdivision $0 \leq s_{0}^{n} \leq s_{1}^{n} \leq \ldots \leq s_{k(n)}^{n}=t$ such that

$$
\lim _{n \rightarrow+\infty} \sup _{1 \leq i \leq k(n)}\left(s_{i}^{n}-s_{i-1}^{n}\right)=0
$$

and

$$
\left\{\begin{array}{l}
\lim _{n \rightarrow+\infty} \sum_{i=1}^{k(n)}\left\|\left(s_{i}^{n}-s_{i-1}^{n}\right) \nabla_{\alpha} \dot{g}\left(s_{i}^{n}\right)-\int_{s_{i-1}^{n}}^{s_{i}^{n}} \nabla_{\alpha} \dot{g}(\tau) d \tau\right\|_{L^{p}\left(\omega ; \mathbb{R}^{3 \times 2}\right)}=0 \\
\lim _{n \rightarrow+\infty} \sum_{i=1}^{k(n)}\left|\left(s_{i}^{n}-s_{i-1}^{n}\right) \theta\left(s_{i}^{n}\right)-\int_{s_{i-1}^{n}}^{s_{i}^{n}} \theta(\tau) d \tau\right|=0 .
\end{array}\right.
$$

For all $s \in\left(s_{i}^{n}, s_{i+1}^{n}\right]$, we define

$$
u_{n}(s):=u\left(s_{i+1}^{n}\right), \quad \text { and } \Psi_{n}(s):=\rho\left(s_{i}^{n}, s_{i+1}^{n}\right) \int_{s_{i}^{n}}^{s_{i+1}^{n}} \nabla_{\alpha} \dot{g}(\tau) d \tau .
$$

As $\nabla_{\alpha} \dot{g} \in L^{1}\left(0, T ; L^{p}\left(\omega^{\prime} ; \mathbb{R}^{3 \times 2}\right)\right)$, we have

$$
\left\|\Psi_{n}(s)\right\|_{L^{p}\left(\omega^{\prime} ; \mathbb{R}^{3 \times 2}\right)} \rightarrow 0,
$$

uniformly with respect to $s \in[0, t]$. In (5.19), we replace $s$ by $s_{i}^{n}$ and $t$ by $s_{i+1}^{n}$, then a summation for $i=0$ to $k(n)-1$ yields

$$
\mathcal{E}(t)-\mathcal{E}(0) \geq 2 \int_{0}^{t} \int_{\omega} \partial\left(\mathcal{Q} W_{0}\right)\left(\nabla_{\alpha} u_{n}(\tau)+\Psi_{n}(\tau)\right) \cdot \nabla_{\alpha} \dot{g}(\tau) d x_{\alpha} d \tau
$$


From (5.21) and Lemma 4.9 in [13], we have for a.e. $\tau \in(0, t)$,

$$
\left|\int_{\omega} \partial\left(\mathcal{Q} W_{0}\right)\left(\nabla_{\alpha} u_{n}(\tau)+\Psi_{n}(\tau)\right) \cdot \nabla_{\alpha} \dot{g}(\tau) d x_{\alpha}-\int_{\omega} \partial\left(\mathcal{Q} W_{0}\right)\left(\nabla_{\alpha} u_{n}(\tau)\right) \cdot \nabla_{\alpha} \dot{g}(\tau) d x_{\alpha}\right| \rightarrow 0 .
$$

Thus, according to (2.2) together with Lebesgue's Dominated Convergence Theorem,

$$
\int_{0}^{t}\left|\int_{\omega} \partial\left(\mathcal{Q} W_{0}\right)\left(\nabla_{\alpha} u_{n}(\tau)+\Psi_{n}(\tau)\right) \cdot \nabla_{\alpha} \dot{g}(\tau) d x_{\alpha}-\int_{\omega} \partial\left(\mathcal{Q} W_{0}\right)\left(\nabla_{\alpha} u_{n}(\tau)\right) \cdot \nabla_{\alpha} \dot{g}(\tau) d x_{\alpha}\right| d \tau \rightarrow 0 .
$$

Thus,

$$
\mathcal{E}(t)-\mathcal{E}(0) \geq \limsup _{n \rightarrow+\infty} 2 \int_{0}^{t} \int_{\omega} \partial\left(\mathcal{Q} W_{0}\right)\left(\nabla_{\alpha} u_{n}(\tau)\right) \cdot \nabla_{\alpha} \dot{g}(\tau) d x_{\alpha} d \tau
$$

But in view of (5.20), (2.2) and Hölder's inequality,

$$
\begin{aligned}
& \sum_{i=1}^{k(n)}\left|\int_{\omega} \partial\left(\mathcal{Q} W_{0}\right)\left(\nabla_{\alpha} u\left(s_{i}^{n}\right)\right) \cdot\left(\left(s_{i}^{n}-s_{i-1}^{n}\right) \nabla_{\alpha} g\left(s_{i}^{n}\right)-\int_{s_{i-1}^{n}}^{s_{i}^{n}} \nabla_{\alpha} \dot{g}(\tau) d \tau\right) d x_{\alpha}\right| \\
\leq & C\left(1+\left\|\nabla_{\alpha} u\right\|_{L^{\infty}\left(0, t ; L^{p}\left(\omega ; \mathbb{R}^{3 \times 2}\right)\right)}^{p-1}\right) \sum_{i=1}^{k(n)}\left\|\left(s_{i}^{n}-s_{i-1}^{n}\right) \nabla_{\alpha} \dot{g}\left(s_{i}^{n}\right)-\int_{s_{i-1}^{n}}^{s_{i}^{n}} \nabla_{\alpha} \dot{g}(\tau) d \tau\right\|_{L^{p}\left(\omega ; \mathbb{R}^{3 \times 2}\right)} \\
& \rightarrow 0,
\end{aligned}
$$

thus, using again (5.20) and (5.12),

$$
\begin{aligned}
\mathcal{E}(t)-\mathcal{E}(0) & \geq 2 \limsup _{n \rightarrow+\infty} \sum_{i=1}^{k(n)}\left(s_{i}^{n}-s_{i-1}^{n}\right) \int_{\omega} \partial\left(\mathcal{Q} W_{0}\right)\left(\nabla_{\alpha} u\left(s_{i}^{n}\right)\right) \cdot \nabla_{\alpha} \dot{g}\left(s_{i}^{n}\right) d x_{\alpha} \\
& =2 \int_{0}^{t} \int_{\omega} \partial\left(\mathcal{Q} W_{0}\right)\left(\nabla_{\alpha} u(\tau)\right) \cdot \nabla_{\alpha} \dot{g}(\tau) d x_{\alpha} d \tau
\end{aligned}
$$

It now remains to show that the inequality proved in Lemma 5.7 is actually an equality. This is the object of the following Lemma.

Lemma 5.8. For every $t \in[0, T]$,

$$
\mathcal{E}(t) \leq \mathcal{E}(0)+2 \int_{0}^{t} \int_{\omega} \partial\left(\mathcal{Q} W_{0}\right)\left(\nabla_{\alpha} u(\tau)\right) \cdot \nabla_{\alpha} \dot{g}(\tau) d x_{\alpha} d \tau
$$

Proof. According to (5.3) and (5.14),

$$
\begin{aligned}
\liminf _{n_{t} \rightarrow+\infty} \mathcal{E}_{\varepsilon_{n_{t}}}(t)= & \liminf _{n_{t} \rightarrow+\infty}\left[\int_{\Omega} W\left(\nabla_{\alpha} u^{\varepsilon_{n_{t}}}(t) \mid \frac{1}{\varepsilon_{n_{t}}} \nabla_{3} u^{\varepsilon_{n_{t}}}(t)\right) d x\right. \\
& \left.\quad+\int_{\Gamma^{\varepsilon_{n}}(t)}\left|\left(\left(\nu_{\Gamma^{n_{n}}}(t)\right)_{\alpha} \mid \frac{1}{\varepsilon_{n_{t}}}\left(\nu_{\Gamma^{\varepsilon_{n}}(t)}\right)_{3}\right)\right| d \mathcal{H}^{2}\right] \\
\geq & 2 \int_{\omega} \mathcal{Q} W_{0}\left(\nabla_{\alpha} u(t)\right) d x_{\alpha}+2 \mathcal{H}^{1}(\gamma(t)) \\
= & \mathcal{E}(t) .
\end{aligned}
$$


On the other hand, by Lemma 5.2, (5.4), (5.5) and (5.12) we have

$$
\begin{aligned}
\limsup _{n_{t} \rightarrow+\infty} \mathcal{E}_{\varepsilon_{n_{t}}}(t) & \leq \limsup _{n \rightarrow+\infty} \mathcal{E}_{\varepsilon_{n}}(t) \\
& \leq \lim _{n \rightarrow+\infty} \mathcal{E}_{\varepsilon_{n}}(0)+\limsup _{n \rightarrow+\infty} \int_{0}^{t} \theta_{n}(\tau) d \tau \\
& =\mathcal{E}(0)+\int_{0}^{t} \theta(\tau) d \tau \\
& =\mathcal{E}(0)+\int_{0}^{t} \int_{\omega} \partial\left(\mathcal{Q} W_{0}\right)\left(\nabla_{\alpha} u(\tau)\right) \cdot \nabla_{\alpha} \dot{g}(\tau) d x_{\alpha} d \tau
\end{aligned}
$$

Accordingly, relations (5.22) and (5.23) complete the proof of the Lemma.

By virtue of Lemmas 5.7 and 5.8, the two-dimensional total energy $\mathcal{E}(t)$ is absolutely continuous with respect to the time $t$ and

$$
\mathcal{E}(t)=\mathcal{E}(0)+2 \int_{0}^{t} \int_{\omega} \partial\left(\mathcal{Q} W_{0}\right)\left(\nabla_{\alpha} u(\tau)\right) \cdot \nabla_{\alpha} \dot{g}(\tau) d x_{\alpha} d \tau
$$

hence, $(u(t), \gamma(t))$ is a quasistatic evolution relative to the boundary data $g(t)$. Let us show now that the three-dimensional bulk and surface energies are converging towards the two-dimensional bulk and surface energies respectively. Note that the following convergence result holds for a subsequence $\left\{\varepsilon_{n}\right\}$ independent of $t$ unlike in Lemma 5.5 where we stated the convergence of the volume energy for a $t$-dependent subsequence $\left\{\varepsilon_{n_{t}}\right\}$.

Lemma 5.9. For every $t \in[0, T]$,

$$
\left\{\begin{array}{l}
\int_{\Omega} W\left(\nabla_{\alpha} u^{\varepsilon_{n}}(t) \mid \frac{1}{\varepsilon_{n}} \nabla_{3} u^{\varepsilon_{n}}(t)\right) d x \rightarrow 2 \int_{\omega} \mathcal{Q} W_{0}\left(\nabla_{\alpha} u(t)\right) d x_{\alpha}, \\
\int_{\Gamma^{\varepsilon_{n}}(t)}\left|\left(\left(\nu_{\Gamma^{\varepsilon_{n}}(t)}\right)_{\alpha} \mid \frac{1}{\varepsilon_{n}}\left(\nu_{\Gamma^{\varepsilon_{n}}(t)}\right)_{3}\right)\right| d \mathcal{H}^{2} \rightarrow 2 \mathcal{H}^{1}(\gamma(t)) .
\end{array}\right.
$$

In particular, $\mathcal{E}_{\varepsilon_{n}}(t) \rightarrow \mathcal{E}(t)$.

Proof. For $t=0$, the result is already proved in Lemma 5.2. Assume now that $t \in(0, T]$ and let $\left\{n_{j}\right\}$ be a $t$-dependent subsequence such that

$$
\liminf _{n \rightarrow+\infty} \int_{\Omega} W\left(\nabla_{\alpha} u^{\varepsilon_{n}}(t) \mid \frac{1}{\varepsilon_{n}} \nabla_{3} u^{\varepsilon_{n}}(t)\right) d x=\lim _{j \rightarrow+\infty} \int_{\Omega} W\left(\nabla_{\alpha} u^{\varepsilon_{n_{j}}}(t) \mid \frac{1}{\varepsilon_{n_{j}}} \nabla_{3} u^{\varepsilon_{n_{j}}}(t)\right) d x .
$$

Arguing as in the proofs of Lemmas 5.1 and 5.5, we can suppose that, for a subsequence of $n_{j}$ (still denoted by $\left.n_{j}\right), u^{\varepsilon_{n_{j}}}(t) \rightarrow u^{*}(t)$ in $S B V^{p}\left(\Omega^{\prime} ; \mathbb{R}^{3}\right)$ for some $u^{*}(t) \in S B V^{p}\left(\omega^{\prime} ; \mathbb{R}^{3}\right)$ with $u^{*}(t)=g(t)$ a.e. on $\omega^{\prime} \backslash \bar{\omega}, S\left(u^{*}(t)\right) \widetilde{\subset} \gamma(t)$, and which is also a minimizer of

$$
v \mapsto 2 \int_{\omega} \mathcal{Q} W_{0}\left(\nabla_{\alpha} v\right) d x_{\alpha}+2 \mathcal{H}^{1}(S(v) \backslash \gamma(t))
$$

among $\left\{v \in S B V^{p}\left(\omega^{\prime} ; \mathbb{R}^{3}\right): \quad v=g(t)\right.$ a.e. on $\left.\omega^{\prime} \backslash \bar{\omega}\right\}$. Hence,

$$
\int_{\omega} \mathcal{Q} W_{0}\left(\nabla_{\alpha} u^{*}(t)\right) d x_{\alpha}=\int_{\omega} \mathcal{Q} W_{0}\left(\nabla_{\alpha} u(t)\right) d x_{\alpha} .
$$


According to Remark 3.2 and Theorem 5.29 in [2],

$$
\begin{aligned}
2 \int_{\omega} \mathcal{Q} W_{0}\left(\nabla_{\alpha} u^{*}(t)\right) d x_{\alpha} & \leq \liminf _{j \rightarrow+\infty} \int_{\Omega} \mathcal{Q} W_{0}\left(\nabla_{\alpha} u^{\varepsilon_{n_{j}}}(t)\right) d x \\
& \leq \lim _{j \rightarrow+\infty} \int_{\Omega} W\left(\nabla_{\alpha} u^{\varepsilon_{n_{j}}}(t) \mid \frac{1}{\varepsilon_{n_{j}}} \nabla_{3} u^{\varepsilon_{n_{j}}}(t)\right) d x .
\end{aligned}
$$

Thus, (5.24) and (5.25) imply that

$$
2 \int_{\omega} \mathcal{Q} W_{0}\left(\nabla_{\alpha} u(t)\right) d x_{\alpha} \leq \liminf _{n \rightarrow+\infty} \int_{\Omega} W\left(\nabla_{\alpha} u^{\varepsilon_{n}}(t) \mid \frac{1}{\varepsilon_{n}} \nabla_{3} u^{\varepsilon_{n}}(t)\right) d x
$$

which ensure together with (5.16) the convergence of the bulk energy i.e.

$$
\int_{\Omega} W\left(\nabla_{\alpha} u^{\varepsilon_{n}}(t) \mid \frac{1}{\varepsilon_{n}} \nabla_{3} u^{\varepsilon_{n}}(t)\right) d x \rightarrow 2 \int_{\omega} \mathcal{Q} W_{0}\left(\nabla_{\alpha} u(t)\right) d x_{\alpha} .
$$

But in view of (2.6), (5.5) and Lemmas 5.2 and 5.5,

$$
\begin{aligned}
\limsup _{n \rightarrow+\infty} \mathcal{E}_{\mathcal{E}_{n}}(t) & =\limsup _{n \rightarrow+\infty} \mathcal{E}_{\varepsilon_{n}}(0)+\limsup _{n \rightarrow+\infty} \int_{0}^{t} \theta_{n}(\tau) d \tau \\
& \leq \mathcal{E}(0)+\int_{0}^{t} \theta(\tau) d \tau \\
& =\mathcal{E}(0)+2 \int_{0}^{t} \int_{\omega} \partial\left(\mathcal{Q} W_{0}\right)\left(\nabla_{\alpha} u(\tau)\right) \cdot \nabla_{\alpha} \dot{g}(\tau) d x_{\alpha} d \tau \\
& =\mathcal{E}(t) .
\end{aligned}
$$

Thus (5.26) and (5.27) yield

$$
\limsup _{n \rightarrow+\infty} \int_{\Gamma^{\varepsilon_{n}(t)}}\left|\left(\left(\nu_{\Gamma^{\varepsilon_{n}}(t)}\right)_{\alpha} \mid \frac{1}{\varepsilon_{n}}\left(\nu_{\Gamma^{\varepsilon_{n}}(t)}\right)_{3}\right)\right| d \mathcal{H}^{2} \leq 2 \mathcal{H}^{1}(\gamma(t))
$$

which, together with (5.3), gives the convergence of the surface term

$$
\int_{\Gamma^{\varepsilon_{n}}(t)}\left|\left(\left(\nu_{\Gamma^{\varepsilon_{n}}(t)}\right)_{\alpha} \mid \frac{1}{\varepsilon_{n}}\left(\nu_{\Gamma^{\varepsilon_{n}}(t)}\right)_{3}\right)\right| d \mathcal{H}^{2} \rightarrow 2 \mathcal{H}^{1}(\gamma(t)) .
$$

Acknowledgments. The author is indebted to Gilles Francfort for having proposed him this problem and for his fruitful suggestions and comments. He also wishes to thank the referee for his remarks and improvements.

\section{References}

[1] L. Ambrosio: Existence theory for a new class of variational problems, Arch. Rational Mech. Anal. 111 (1990) 291-322.

[2] L. Ambrosio, N. Fusco and D. Pallara: Functions of bounded variation and free discontinuity problems, Oxford University Press, Oxford (2000). 
JEAN-FranÇOIS BABADJiAN

[3] J.-F. Babadjian: Réduction dimensionnelle pour des milieux hétérogènes, troués ou fissurés, $\mathrm{PhD}$ thesis, University Paris 13 (2005).

[4] J. M. Ball, B. Kirchheim and J. Kristensen: Regularity of quasiconvex envelopes, Calc. Var. Partial Differential Equations 11 (2000) 333-359.

[5] K. Bhattacharya, I. Fonseca and G. A. Francfort: An asymptotic study of the debonding of thin films, Arch. Rational Mech. Anal. 161 (2002) 205-229.

[6] G. Bouchitté, I. Fonseca, G. Leoni and L. Mascarenhas: A global method for relaxation in $W^{1, p}$ and in $S B V_{p}$, Arch. Rational Mech. Anal. 165 (2002) 187-242.

[7] A. Braides and V. Chiadò Piat: Integral representation results for functionals defined on $S B V\left(\Omega ; \mathbb{R}^{m}\right)$, J. Math. Pures Appl. 75 (1996) 595-626.

[8] A. Braides, A. Defranceschi and E. Vitali: Homogenization of free discontinuity problems, Arch. Rational Mech. Anal. 135 (1996) 297-356.

[9] A. Braides and I. Fonseca: Brittle thin films, Appl. Math. Optim. 44 (2001) 299-323.

[10] A. Braides, I. Fonseca and G. A. Francfort: 3D-2D Asymptotic analysis for inhomogeneous thin films, Indiana Univ. Math. J. 49 (2000) 1367-1404.

[11] B. Dacorogna: Direct methods in the calculus of variations, Springer-Verlag, Berlin (1989).

[12] G. Dal Maso: An introduction to $\Gamma$-convergence, Birkhäuser, Boston (1993).

[13] G. Dal Maso, G. A. Francfort and R. Toader: Quasi-static crack growth in finite elasticity, Arch. Rational Mech. Anal. 176 (2005) 165-225.

[14] G. Dal Maso, G. A. Francfort and R. Toader: Quasi-static evolution in brittle fracture : the case of bounded solutions, Calculus of Variations : Topics from the Mathematical Heritage of E. De Giorgi. Quaderni di Matematica 14 (2005) 247-265.

[15] I. Ekeland and R. Temam: Analyse convexe et problèmes variationnels, Dunod, Gauthiers-Villars, Paris (1974).

[16] L. C. Evans and R. F. Gariepy: Measure theory and fine properties of functions, Boca Raton, CRC Press (1992).

[17] I. Fonseca and G. A. Francfort: Relaxation in $B V$ versus quasiconvexification in $W^{1, p}$; a model for the interaction between fracture and damage, Calc. Var. Partial Differential Equations 3 (1995) 407-446.

[18] G. A. Francfort and C. J. Larsen: Existence and convergence for quasistatic evolution in brittle fracture, Comm. Pure Appl. Math. 56 (2003) 1465-1500.

[19] G. A. Francfort and J.-J. Marigo: Revisiting brittle fracture as an energy minimization problem, $J$. Mech. Phys. Solids 46 (1998) 1319-1342.

[20] A. Giacomini: Ambrosio-Tortorelli approximation of quasi-static evolution of brittle fracture, Calc. Var. Partial Differential Equations 22 (2005) 129-172.

[21] A. Giacomini and M. Ponsiglione: A $\Gamma$-convergence approach to stability of unilateral minimality properties in fracture mechanics and applications, Preprint SISSA (2004). 
QUASistatic EVOLUTION OF A BRITTLE THIN FILM

[22] H. Le Dret and A. Raoult: The nonlinear membrane model as variational limit of nonlinear threedimensional elasticity, J. Math. Pures Appl. 74 (1995) 549-578.

Jean-François Babadjian

L.P.M.T.M., Université Paris Nord, 93430, Villetaneuse, France

E-mail address: jfb@galilee.univ-paris13.fr 